\title{
TOPOLOGICAL ANALYSIS OF SYNTACTIC STRUCTURES
}

\author{
ALEXANDER PORT, TAELIN KARIDI, MATILDE MARCOLLI
}

\begin{abstract}
We use the persistent homology method of topological data analysis and dimensional analysis techniques to study data of syntactic structures of world languages. We analyze relations between syntactic parameters in terms of dimensionality, of hierarchical clustering structures, and of non-trivial loops. We show there are relations that hold across language families and additional relations that are family-specific. We then analyze the trees describing the merging structure of persistent connected components for languages in different language families and we show that they partly correlate to historical phylogenetic trees but with significant differences. We also show the existence of interesting non-trivial persistent first homology groups in various language families. We give examples where explicit generators for the persistent first homology can be identified, some of which appear to correspond to homoplasy phenomena, while others may have an explanation in terms of historical linguistics, corresponding to known cases of syntactic borrowing across different language subfamilies.
\end{abstract}

\section{INTRODUCTION}

The use of methods of Topological Data Analysis in linguistics was introduced in [35], where it was shown that the persistent homology of the SSWL data of syntactic structures of world languages reveals the presence of additional structures in the data, such as persistent first homology, that are not identifiable via other more traditional methods of computational measurements of language relatedness.

In the present paper we carry out a much more in depth analysis, using topological methods, of the data of syntactic structures (both from the SSWL database [44 and from the data of syntactic parameters collected by Longobardi and collaborators of the LanGeLin project, [22], 20]).

When we look at the data points as syntactic features or syntactic parameters, with coordinates given by the values of the parameter over a given set of languages, we focus on the question of identifying relations between these syntactic variables. This is a main open question already investigated by other methods in [18, [31, [33], 338. We analyze the clustering structure between syntactic parameters by analyzing the persistent connected components of the data at various scales and the resulting tree that follows the order in which the components merge as the scale parameter increases. We compare the detected cluster structure obtained in this way with those discussed in [18 and [31. We also compute the persistent $H_{1}$ and we show that there are further relations between syntactic parameters corresponding to non-trivial persistent $H_{1}$-generators that are not detectable by cluster information. Moreover, we also compute estimates of dimensionality of these data sets. We compare the dimensionality analysis for balls and spheres of varying dimensions with the dimensionality analysis of our sets of data point of syntactic parameters and we identify peaks around the most likely dimension estimates. We also perform dimension estimates for the data of syntactic parameters with coordinates evaluated only over certain language 
families, in order to detect the presence of relations between syntactic parameters that may be language family specific and not universal across families. We find that indeed the dimension drops when coordinates are restricted to subfamilies indicating the presence of family-specific relations between the syntactic variables in addition to universal ones.

When we look at the data points as languages, with coordinates given by the values of their syntactic parameters (LanGeLin data) or binary syntactic variables (SSWL), we focus on three main questions. The first question is to what extent the persistent $H_{0}$ (the persistent connected components) of the data set can be used as an alternative method for the reconstruction of phylogenetic trees of language families. In [35], based on some preliminary cases of topological analysis of syntactic structures, we conjectured that the barcode diagram of the persistent connected components could provide a reliable reconstruction of the phylogenetic tree of the languages involved. In the more detailed analysis that we present here we show that, while this is indeed sometimes the case, and the trees derived from the merging and clustering structure of the persistent connected components correlate with the phylogenetic trees of language families in terms of grouping together or languages by subfamilies, the information contained in the persistent components trees and in the phylogenetic trees is not always identical. We will show examples where the structure of the tree of the persistent connected components significantly differs from the phylogenetic tree while still retaining much of the information on the grouping into subfamilies. Investigating the discrepancies between phylogenetic trees and persistent components trees will provide better insight on what information about language relatedness is captured by the persistent connected components that differs from historical family relatedness. The main information carried by persistent components trees is a hierarchical organization of the spreading of syntactic features across languages.

The second question we investigate is the detection of higher dimensional topological structures, in particular the persistent first homology group $H_{1}$, and the meaning of the resulting structures from the point of view of historical linguistics. Unlike typical random simplicial complexes, the Vietoris-Rips complexes at varying scales associated to data of syntactic parameters tend to exhibit no higher dimensional homology, that is, no nontrivial persistent generators of the $H_{k}$ homology groups for $k \geq 2$. This seems to indicate that passing from trees to networks given by more general graphs (to account for the presence of non-trivial persistent $H_{1}$ homology) suffices to describe relatedness between languages, without the need to introduce higher dimensional geometries. The structure of the persistent $H_{1}$ that we see in the case of syntactic data also differs from random simplicial sets in the fact that non-trivial $H_{1}$-generators appear only in the larger clusters, and there is less overlap between them in the barcode diagram than expected in a random setting. As we will show in specific examples, non-trivial generators of the persistent $H_{1}$ homology can sometimes consist of a set of languages not belonging to the same subfamilies, but for which it is known that there have been historical interactions and possible influences at the syntactic level (for example between the Hellenic and the Slavic languages). In other cases the set of languages that provide explicit $H_{1}$-generators do not seem to be plausibly related through historical influence that could lead to borrowing at the syntactic level. There are two possible explanation for this second type of $H_{1}$-generator. Either the $H_{1}$-generator is not the historically relevant one and a homologous one (differing by a boundary and still generating the same $H_{1}$-class) would be the one that can be interpreted in historical linguistic terms, or else the $H_{1}$-generator is only detecting homoplasy phenomena in syntax. 
In phylogenetics homoplasy refers to those traits that are independently gained in separate branches of the phylogenetic tree and are not due to a common ancestor. Homoplasy phenomena in syntax are observed when languages that are not closely related exhibit syntactic similarities. When such phenomena occur one can expect that an $H_{1}$-generator may appear in the topological analysis where the languages involved have no clear historical record of mutual influence involving the possibility of syntactic borrowing. We will analyze examples of $H_{1}$-generators in a case for which we can propose at least a conjectural historical linguistic explanation and in a case that seems to be due exclusively to homoplasy. While the existence of non-trivial $H_{1}$-generators was already observed in [35], in the analysis of Indo-European language family data from the SSWL syntactic features, in this paper we carry out a much more extensive analysis throughout all the clusters and four different main language families in the SSWL database, as well as for the LanGeLin data, hence we are able to identify several more cases of non-trivial $H_{1}$-generators, also outside of the Indo-European case (for example we show there are clusters where the Niger-Congo languages also exhibit persistent $H_{1}$-generators, see Figure 2, while those sub-clusters of the Niger-Congo family that we had previously analyzed in [35] showed no non-trivial $H_{1}$ ).

The third question is an estimation of dimensionality for different language families as a measure of how spread out the syntactic features are across languages in a given family. We show that language families like the Niger-Congo or the Indo-European family have an estimated dimensionality that significanty exceeds the dimensionality computed over the entire database of languages, while other language families like the Afroasiatic or Austronesian family have an estimated dimensionality that is significantly smaller than that of the full set of languages. We also consider the Ural-Altaic hypothesis from this point of view of dimensionality estimates and we show that the combined estimate for the two sets of languages shows two distinct peaks one closer to the peak for the Altaic languages alone and one closer to that of the Uralic. (This is consistent with recent observations of a similar nature in [21].)

We discuss also the use of the principal components in our data analysis, both in view of identifying possible linguistic interpretations for the different weights assigned to the syntactic parameters by the PCA method and in order to understand the effect that the variance of the PCA can have on the details of the reconstruction of the tree of the persistent components and the $H_{1}$-generators.

1.1. Persistent topology: a quick review. In the field of data analysis researchers often come across very large data sets. The first question that arises when analyzing big data is how to make sense of it. More precisely, this generally means trying to identify certain lower dimensional loci (manifolds or other kinds of geometric and topological objects) the data points lie on (or sufficiently near) inside a much higher dimensional ambient space. In other words, one wants to know what is the "shape" of the data. Topology is the branch of mathematics that studies shapes, hence topological data analysis is especially suited for this task of analyzing high dimensional and complex data sets and identify the topological structures the data exhibit. In particular, persistent homology is a fundamental tool at the heart of topological data analysis, see [7] and also [4], [10], [14, [42]. Persistent homology gained much popularity in recent years as the primary method of topological data analysis and found various applications, ranging from the analysis of protein structures in biology, to analyzing $3 D$ images in image recognition, to computational neuroscience, and more. $\mathrm{A}$ 


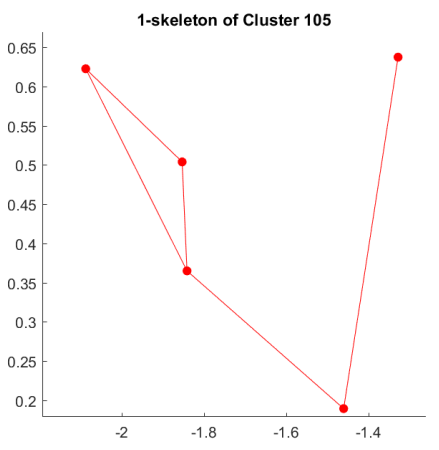

(A) cluster 105

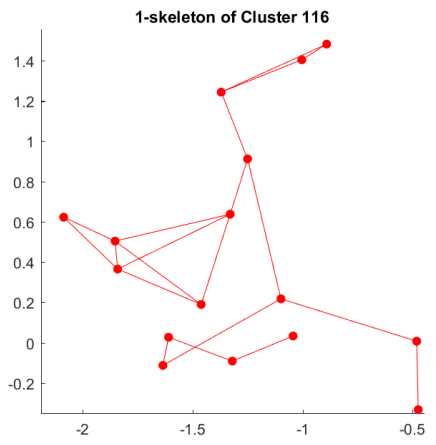

(C) cluster 116

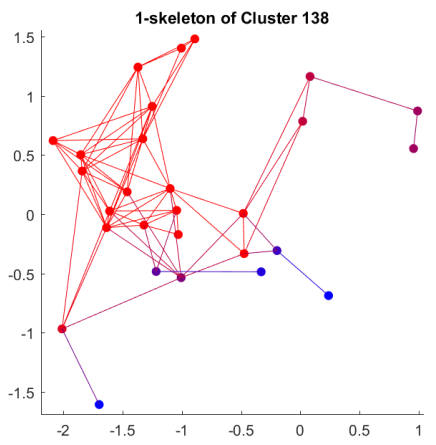

(E) cluster 138

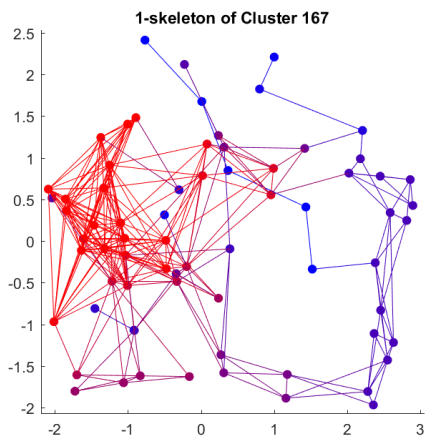

(G) cluster 167

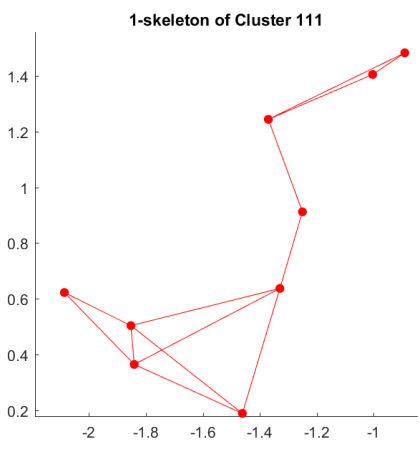

(B) cluster 111

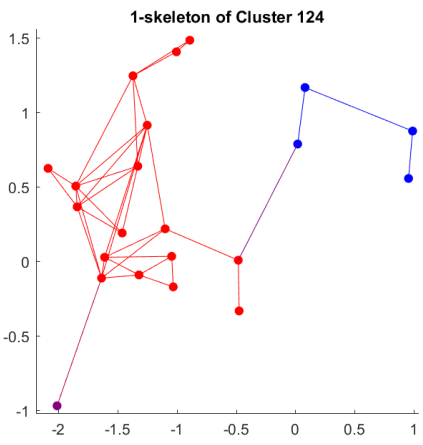

(D) cluster 124

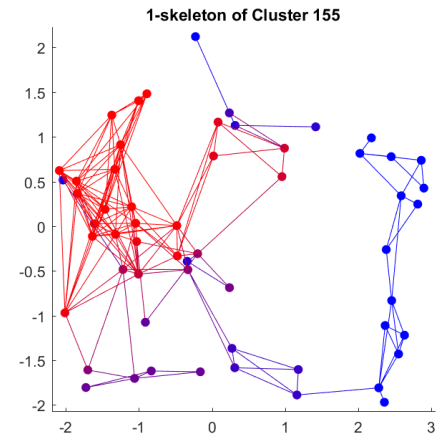

(F) cluster 155

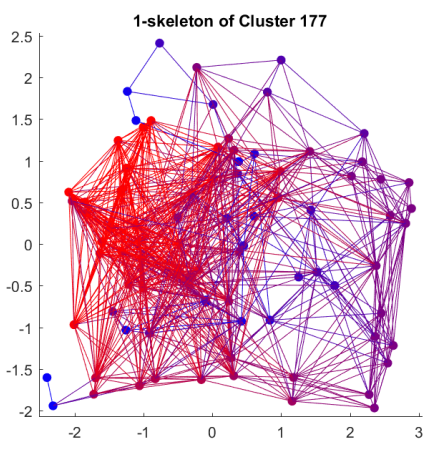

(H) cluster 177

FIGURE 1. 1-skeletons of the Indo-European family in varying radii, SSWL. 
more theoretical and categorical viewpoint on persistent homology was developed in [6], [17], 25]. Barcode diagrams were also previously studied as 'canonical forms' in [2].

More precisely, topological data analysis aims at extracting topological features from big data sets, by computing topological invariant of a corresponding space at various scales. The scale dependence of the topological structures can be used to separate the meaningful characteristics of the underlying space, which are assumed to be persistent over a larger range of scales, from effects caused by noise in the data, which are transitory and only appear within a small range of scales. In order to compute the homology groups at different scales one first constructs a scale-dependent simplicial set or chain-complex from the data points, using a suitable distance function in a large ambient space where the data points are embedded. This construction converts the discrete data set into a global topological object. The scale dependence gives rise to a family of simplicial sets organized as a filtration, where each simplicial set at a given scale is nested into the ones at larger scales. A sampling of this nested family of simplicial sets can be seen, in the case of the data set given by the SSWL database of syntactic parameters, in Figure 1. In the family of simplicial sets so constructed, the scale, which is also referred to as the proximity parameter, ranges between zero and the maximum of the distances between any two points in the data set. At intermediate scales, one considers those subsets of points with mutual distances smaller than the given scale and joins them by simplexes. We review the construction more in detail in Section 1.1.1. From the filtered simplicial complexes we then produce a barcode graph by computing the homology groups at various scales. The barcode graph represents the persistent homology of a chain-complex uniquely, see [7], [42]. Each horizontal line corresponds to a generator of a homology group, where its starting point is the scale of its birth and its ending point is the scale of its death (we will discuss this more in detail below). This barcode graph encodes the information regarding the persistent homology of the data and is beneficial in the task of distinguishing the significant features from the noise.

1.1.1. The Vietoris-Rips Complex. Data sets are a discrete set of points and therefore have a trivial topology. In order to convert the data into a more interesting topological object, one first needs to construct a simplicial complex (in fact a scale-dependent family of simplicial complexes) from the data. Then, for each complex we can compute the corresponding homology groups. Loosely speaking, homology computes the number of $n$-dimensional holes in the shape of the data.

Given a finite set of points $X \subset \mathbb{R}^{N}$ and a scale $\epsilon>0$, the Vietoris-Rips complex $R_{\epsilon}(X)$ over a field $\mathbb{F}$ is an abstract simplicial complex whose space of $n$-simplices $\mathbb{R}_{n}(X, \epsilon)$ is the $\mathbb{F}$-vector space spanned by all the unordered $(n+1)$-tuples $\left\{x_{0}, \ldots, x_{n}\right\}$ in the data set such that for every $0 \leq i \neq j \leq n$ we have $d\left(x_{i}, x_{j}\right) \leq \epsilon$, where $d: \mathbb{R}^{N} \times \mathbb{R}^{N} \rightarrow \mathbb{R}$ is the Euclidean distance function. We have a filtration, i.e. a sequence of nested simplicial complexes with the inclusion maps $R_{n}(X, \epsilon) \rightarrow R_{n+1}(X, \epsilon)$. Moreover, we have the usual boundary maps $\partial_{n}: R_{n}(X, \epsilon) \rightarrow R_{n+1}(X, \epsilon)$ that satisfy the condition $\partial_{n} \circ \partial_{n+1}=0$ for every $n>0$. Therefore $\operatorname{Im}\left(\partial_{n+1}\right) \subseteq \operatorname{ker}\left(\partial_{n}\right)$ so the quotient between $\operatorname{ker}\left(\partial_{n}\right)$ and $\operatorname{Im}\left(\partial_{n+1}\right)$ is well defined. Elements in $\operatorname{ker}\left(\partial_{n}\right)$ are called $n$-cycles and elements in $\operatorname{Im}\left(\partial_{n+1}\right)$ are called $n$-boundaries. Now we can define the $n$-th homology group:

$$
H_{n}(X, \epsilon):=\operatorname{ker}\left(\partial_{n}\right) / \operatorname{Im}\left(\partial_{n+1}\right) .
$$

The dimension of the $n$-th homology, $\beta_{n}(X, \epsilon):=\operatorname{dim}\left(H_{n}(X, \epsilon)\right)=\operatorname{dim}\left(\operatorname{ker}\left(\partial_{n}\right)\right)-$ $\operatorname{dim}\left(\operatorname{Im}\left(\partial_{n+1}\right)\right)$ is called the $n$-th Betti number and counts the number of holes in $X$. For 


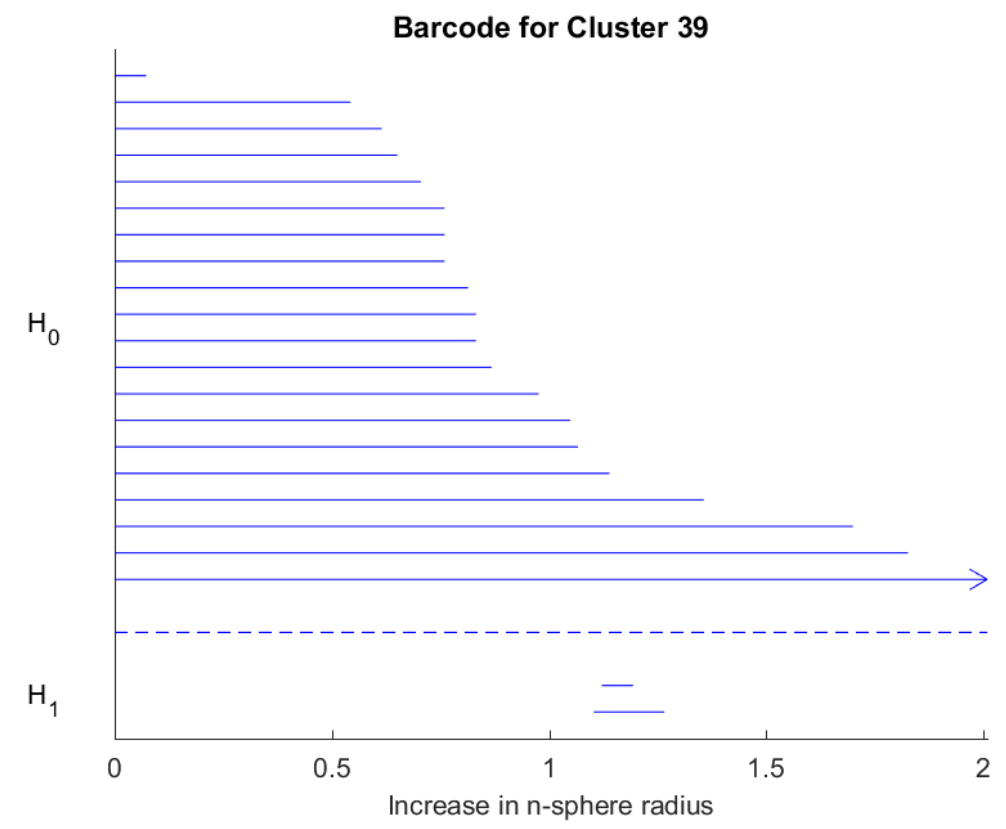

(A) Austronesian

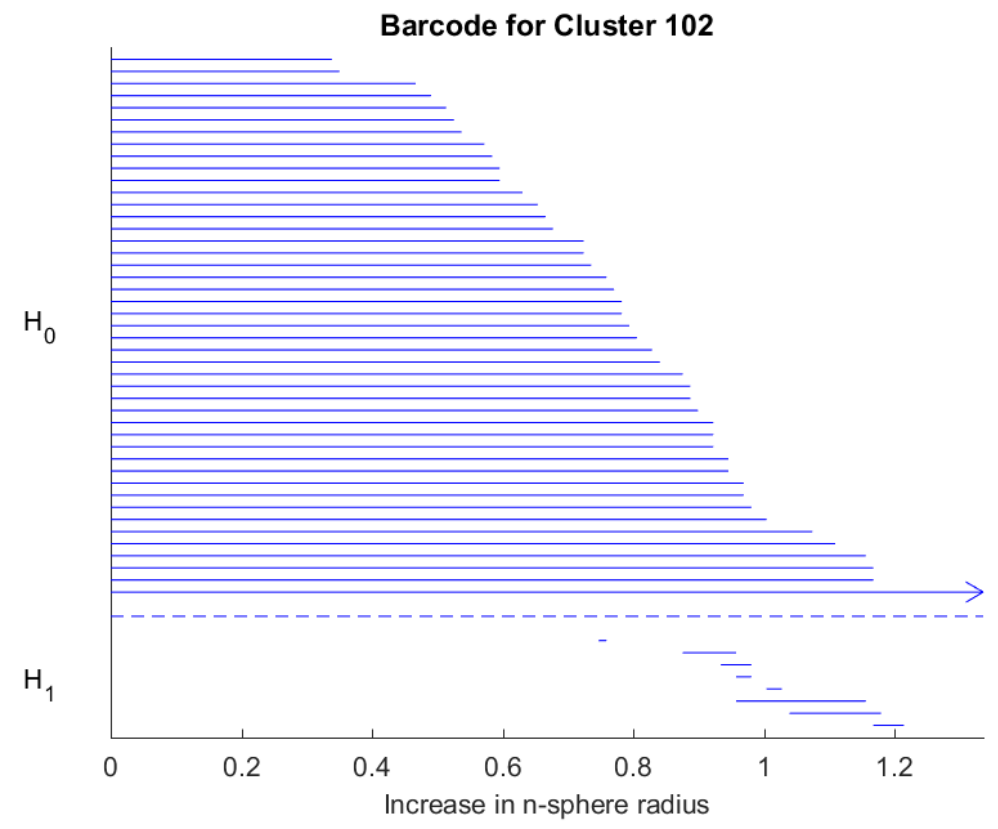

(B) Niger-Congo

Figure 2. Barcode graphs of the Austronesian and Niger-Congo families from the SSWL data set. 
example, $\beta_{0}$ is the number of connected components, $\beta_{1}$ is the number of holes, and $\beta_{2}$ is the number of voids. To summarize, for every $\epsilon$ we can define a sequence of Vitories-Rips complexes, but one might ask himself what is a "good" scale $\epsilon$, or whether there is an ultimate $\epsilon$ in the way that it reveals significant features of the data. In view of this we will define the notion of persistent homology.

1.1.2. Persistent Homology. Let $X \subseteq \mathbb{R}^{n}, \epsilon_{1}<\epsilon_{2}<\cdots<\epsilon_{n}$ and $R\left(X, \epsilon_{1}\right) \subseteq \cdots \subseteq$ $R\left(X, \epsilon_{n}\right)=R(X)$ the corresponding filtered Vietoris-Rips simplicial complex. Note that, by functoriality of homology, for any $1 \leq k \leq n$ the inclusion maps $R\left(X, \epsilon_{i}\right) \hookrightarrow R\left(X, \epsilon_{j}\right)$ induces linear maps $h_{i, j}: H_{k}\left(X, \epsilon_{i}\right) \rightarrow H_{k}\left(X, \epsilon_{j}\right)$ for every $i<j$. The $k$-th persistent homology of $R(X)$ is the pair $\left(H_{k}\left(X, \epsilon_{i}\right)_{1 \leq i \leq n},\left(h_{i, j}\right)_{1 \leq i \leq j \leq n}\right)$.

By computing the homology as the value of the scale $\epsilon$ increases, the persistent homology detects which topological features of the data (i.e non trivial generators of the homology groups) persist over relatively long intervals and therefore are significant.

One way to formalize this property is by considering the range of the maps $h_{\epsilon, \epsilon^{\prime}}$ : $H_{k}(X, \epsilon) \rightarrow H_{k}\left(X, \epsilon^{\prime}\right)$ for $\epsilon<\epsilon^{\prime}$, which detects the part of the homology $H_{k}(X, \epsilon)$ at scale $\epsilon$ that persists at scale $\epsilon^{\prime}$. By adjusting the length of the persistence interval $\left[\epsilon, \epsilon^{\prime}\right]$ one can distinguish between the part of the homology that persists for a longer range of scales (structure) from the part that disappears within a very short range of scales (noise).

There is a visual way to encode this information, called a barcode graph (see Figure 2 for examples taken from the SSWL data of syntactic structures). For every $1 \leq k \leq n$ the number of lines in the graph is the Betti number $\beta_{k}$, where each line corresponds to a non trivial generator of $H_{k}(X)$. There is a line between filtration step $i$ and filtration step $i+1$ if a generator of $H_{k}\left(X, \epsilon_{i}\right)$ is mapped to a non trivial generator of $H_{k}\left(X, \epsilon_{i+1}\right)$, under the map $h_{i, i+1}$ defined earlier. If a non-trivial generator of $H_{k}\left(X, \epsilon_{i}\right)$ is sent to zero in $H_{k}\left(X, \epsilon_{i+1}\right)$, the line ends at $\epsilon_{i+1}$.

1.2. Syntactic structures of world languages. The data we are interested in analyzing encode syntactic structures of different world languages. A fundamental idea in modern linguistics, developed within the setting of Chomsky's Principles and Parameters model [8], [9] postulates that the syntax of any human language can be encoded in a universal set of binary variables, or syntactic parameters. A general introduction to syntactic parameters aimed at readers without a linguistics background can be found in [1]. The main idea is that the syntactic parameters should provide coordinates for the space of possible human languages. The values for the set of syntactic parameters, each viewed as a yes/no answer to whether a certain syntactic construction is possible in a given language, distinguish between different languages. For an overview of the recent state of the art in linguistic research on syntactic parameters, see the collection of papers in the volume [16].

Open questions regarding syntactic parameters that are especially suitable for a mathematical approach include:

- identifying dependencies between parameters, in other words identifying the lower dimensional locus inside the binary space of all possible parameter assignments that is occupied by actual human languages;

- identifying relatedness between languages on the basis of the distribution of their syntactic features. 
With respect to the second question, the use of syntactic parameters as a method for computational reconstruction of phylogenetic trees of language families was developed in [19], [20], [22], 23] and recently also considered from the perspective of phylogenetic algebraic geometry in [37] and from the topological perspective we consider here in [35. The first question has been approached with mathematical methods in [18, [27, [31], [33], [38], [41].

1.2.1. Databases of syntactic structures. The main sources currently available that record binary data of syntactic structures of languages are the SSWL database [44] (Syntactic Structures of World Languages) and the data of Longobardi and the LanGeLin collaboration, [22], 20].

Several of the binary variables recorded in the SSWL database should not be regarded as genuine "syntactic parameters" in the sense that linguists define, although they still encode useful information about syntactic structures. Thus, in the following, since we will include all these variables in our analysis, we will refer more loosely to "syntactic structures" or "binary syntactic variables" instead of using the more specific term "syntactic parameters".

The 116 binary variables recorded in the SSWL database include:

- variables describing word order properties, from 01-Subject Verb to 22-Noun Pronomial Possessor

- variables $A 01-A 04$ describing relations of adjectives to nouns and degree words

- variable AuxSel01 about the selection of auxiliary verbs

- variables $\mathrm{C01-C04}$ related to word order properties of complementarizer and clause and adverbial subordinator and clause

- variables N201-N211 on properties of numerals

- variables $\mathrm{Neg01-Neg14}$ on negation

- variables OrderN301-OrderN312 on word order properties involving demostratives, adjectives, nouns, and numerals

- variables $Q 01-Q 15$ regarding the structure of questions

- variables Q16Nega-Q18Nega and $Q 19 N e g Q-Q 22 N e g Q$ on answers to negative questions

- variables V201-V202 on declarative and interrogative Verb-Second

- variables $w 01 a-w 01 c$ on indefinite mass nouns in object position

- variables w02a-w02c on definite mass nouns in object position

- variables $w 03 a-w 03 d$ on indefinite singular count nouns in object position

- variables $w 04 a-w 04 c$ on definite singular count nouns in object position

- variables $w 05 a-w 05 c$ on indefinite plural count nouns in object position

- variables $w 06 a-w 06 c$ on definite plural count nouns in object position

- variables $w 06 a-w 06 c$ on definite plural count nouns in object position

- variables $w 0 \% a-w 0 \% d$ on nouns with (intrinsically) unique referents in object position

- variables $w 08 a-w 08 d$ on proper names in object position

- variables $w 09 a-w 09 b$ on order of article and proper names in object position

- variables $w 10 a-w 10 c$ on proper names modified by an adjective in object position

- variables $w 11 a-w 11 b$ on order of proper names and adjectives in object position

- variables $w 12 a-w 12 f$ on order of definite articles and nouns in object position

- variables w20a-w20e on singular count nouns in vocative phrases

- variables w21a-w21e on proper nouns in vocative phrases

- variables w22a-w22e on plural nouns in vocative phrases. 
A specific description of each variable is given in the SSWL online site [44] or in the updated Terraling database that SSWL migrated to, [45].

One of the main problems of the SSWL data is the fact that different languages across the database are recorded with very different levels of completeness. While for some languages $100 \%$ of the SSWL binary variables are mapped, other languages have very small percentage of completeness. This problem occurs not only between language families but also within each family. We will discuss later how we deal with this problem of incomplete mapping. This problem does not affect the LanGeLin data.

The data of the LanGeLin collaboration should be regarded a priori as an independent set of data with respect to the features recorded in the SSWL data. The variables in the LanGeLin can be regarded linguistically as genuine syntactic parameters based on the Modularized Global Parameterization approach developed by Longobardi [19], [20]. The LanGeLin data are not strictly binary variables, since they also encode the possibility that some of the parameters may become undefined as an effect of entailment relations from other parameters. Thus, they are encoded as ternary variables with the two main binary states \pm 1 for a syntactic parameter being expressed or not expressed in a given language and a third state 0 to allow for the undefined case. The syntactic parameters recorded in the LanGeLin data are listed in Figure 3 (reproduced from [18]).

1.2.2. Languages and Language Families. The SSWL dataset covers a set of 253 languages. In our analysis here we focus only on certain subsets of languages that belong to some of the main language families. The two families that are best represented in the database are the Indo-European and the Niger-Congo, hence we will be primarily working with these languages. We will also consider two other families with fewer representatives in the SSWL database: the Afro-Asiatic and the Austronesian languages.

- Indo-European: Afrikaans, Albanian, Ancient Greek, Armenian, Bellinzonese, Bengali, Brazilian Portuguese, Breton, Bulgarian, Burgenland-Romani, Calabrian, Catalan, Cimbrian, Croatian, Cypriot Greek, Czech, Danish, Digor Ossetic, Dutch, Eastern Armenian, English, English (Middle), English (Singapore), European Portuguese, Faroese, Farsi, French, French (Ivorian), Frisian, Galician, German, Gothic, Greek, Greek (Calabria, Cappadocian, Homeric, Kydonies, Lesbos, Medieval, Pontic), Haitian Creole, Hindi , Hittite, Icelandic, Irish, Italian, Italian (Napoletano Antico, Old, Reggiano), Iron Ossetic, Jamaican Creole English, Kurdish (Sorani), Latin, Latin (Late), Lithuanian, Middle Dutch, Mòcheno, Neapolitan, Nepali, Norwegian, Occitan, Odia (Oriya), Oevdalian, Old English, Old French, Old Norse, Old Saxon, Panjabi, Pashto, Polish, Portuguese, Romanian, Russian, Sanskrit, Scottish Gaelic, Saramaccan, Serbian, Sicilian, Slovenian, Spanish, Swedish, Swiss German, Teramano, Tocharian A, Tocharian B, Ukrainian, Vlaški-Žejanski-Istro-Romanian, Welsh, West Flemish, Western Armenian, Yiddish.

- Niger-Congo: Agni Indénié, Akan-twi, Babanki, Bafut, Bambara, Bandial, Baoulé, Basaa, Baule-Kôdeh, Beng, Chichewa, Dagaare, Digo, Ewe, Ewondo, Farefari, Fe'efe'e, Ga, Ghomálá', Guébie, Gungbe (Porto-Novo), Gurene, Hanga, Ibibio, Igala, Igbo, Ijo (Kaiama), Kenyang, KiLega, Kinande, Kindendeule, Kiswahili, Kiyaka, Kom, Konni, Koyo, Kusaal, Lubukusu, Mada, Mankanya, Medumba, Naki, Nawdm, Nda'nda', Ndut, Nkore-Kiga, Nupe, Nweh, Olukumi, Oluwanga, Oluwanga 


\begin{tabular}{|c|c|c|c|}
\hline FGP & gramm. person & GSI & grammaticalised inalienability \\
\hline FGM & gramm. Case & ALP & alienable possession \\
\hline FPC & gramm. perception & GST & grammaticalised Genitive \\
\hline FGT & gramm. temporality & GEI & Genitive inversion \\
\hline FGN & gramm. number & GNR & non-referential head marking \\
\hline GCO & gramm. collective number & STC & structured cardinals \\
\hline PLS & plurality spreading & GPC & gender polarity cardinals \\
\hline FND & number in $\mathrm{D}$ & PMN & personal marking on numerals \\
\hline FSN & feature spread to $\mathrm{N}$ & $\mathrm{CQU}$ & cardinal quantifiers \\
\hline FNN & number on $\mathrm{N}$ & PCA & number spread through cardinal adjectives \\
\hline SGE & semantic gender & PSC & number spread from cardinal quantifiers \\
\hline FGG & gramm. gender & RHM & Head-marking on Rel \\
\hline CGB & unbounded sg N & FRC & verbal relative clauses \\
\hline DGR & gramm. amount & NRC & nominalised relative clause \\
\hline DGP & gramm. text anaphora & NOR & NP over verbal relative clauses/ \\
\hline CGR & strong amount & & adpositional genitives \\
\hline NSD & strong person & AER & relative extrap. \\
\hline FVP & variable person & ARR & free reduced rel \\
\hline DGD & gramm. distality & DOR & def on relatives \\
\hline DPQ & free null partitive Q & NOD & NP over D \\
\hline$\overline{\mathrm{DCN}}$ & article-checking $\mathrm{N}$ & $\bar{N} \mathrm{NOP}$ & NP over non-genitive arguments \\
\hline DNN & null-N-licensing art & PNP & P over complement \\
\hline DIN & D-controlled infl. on $\mathrm{N}$ & NPP & N-raising with obl. pied-piping \\
\hline FGC & gramm. classifier & NGO & $\mathrm{N}$ over GenO \\
\hline DBC & strong classifier & NOA & N over As \\
\hline $\mathrm{XCN}$ & conjugated nouns & NM2 & $\mathrm{N}$ over M2 As \\
\hline GSC & c-selection & NM1 & N over M1 As \\
\hline NOE & $\mathrm{N}$ over ext. arg. & EAF & fronted high As \\
\hline HMP & NP-heading modifier & NON & $\mathrm{N}$ over numerals \\
\hline AST & structured APs & FPO & feature spread to genitive postpositions \\
\hline FFS & feature spread to struct. APs & ACM & class MOD \\
\hline ADI & D-controlled infl. on A & DOA & def on all $+\mathrm{N}$ \\
\hline DMP & def matching pron. poss. & NEX & gramm. expletive article \\
\hline DMG & def matching genitives & NCL & clitic poss. \\
\hline GCN & Poss $^{\circ}$-checking $\mathrm{N}$ & PDC & article-checking poss. \\
\hline GFN & Gen-feature spread to Poss ${ }^{\circ}$ & ACL & enclitic poss. on As \\
\hline GAL & Dependent Case in NP & APO & adjectival poss. \\
\hline GUN & uniform Gen & WAP & wackernagel adjectival poss. \\
\hline EZ1 & generalized linker & AGE & adjectival Gen \\
\hline EZ2 & non-clausal linker & OPK & obligatory possessive with kinship nouns \\
\hline EZ3 & non-genitive linker & TSP & split deictic demonstratives \\
\hline GAD & adpositional Gen & TSD & split demonstratives \\
\hline GFO & $\mathrm{GenO}$ & TAD & adjectival demonstratives \\
\hline PGO & partial GenO & TDC & article-checking demonstratives \\
\hline GFS & GenS & TLC & Loc-checking demonstratives \\
\hline GIT & Genitive-licensing iterator & TNL & NP over Loc \\
\hline
\end{tabular}

Figure 3. List of the LanGeLin syntactic parameters. 
(Eji), Shupamem, Tommo-So, Tuki (Tukombo), Twi, Vata, Wan, Wolof, Xhosa, Yémba, Yoruba, Zulu.

- Austronesian: Acehnese, Atayal (Squliq), Bajau (West Coast), Fijian, Ilokano, Indonesian, Isbukun Bunun, Kayan, Malagasy, Maori, Marshallese, Niuean, Palue, Papuan Malay, Samoan, Sasak, Tagalog, Titan, Tongan, Tukang Besi, Zamboageño Chabacano.

- Afro-Asiatic: Amharic, Arabic (Gulf), Bole, Egyptian Arabic, Hausa, Hebrew, Hebrew (Biblical), Lebanese Arabic, Mbuko, Miya, Moroccan Arabic, Muyang, Senaya, Tigre, Wolane.

The list of languages included in the LanGeLin data is given by: Kadiweu (Ka), Kuikuro $(\mathrm{Ku})$, Ragusa (RGS), Mussomeli (MuS), Aidone (AdS), Northern Calabrese (NCa), Southern Calabrese (SCa), Salentino (Sal), Campano (Cam), Italian (It), Spanish (Sp), French (Fr), Portuguese (Ptg), Romanian (Rm), Latin (Lat), Classical Greek (CIG), New Testament Greek (NTG), Salento Greek (SaG), Calabrian Greek A (CGA), Calabrian Greek B (CGB), Greek (Grk), Romeyka Pontic Greek (RPG), Cypriot Greek (CyG), Gothic (Got), Old English (OE), English (E), German (D), Danish (Da), Icelandic (Ice), Norwegian (Nor), Bulgarian (Blg), Serb-Croatian (SC), Slovenian (Slo), Polish (Po), Russian (Rus), Irish (Ir), Welsh (Wel), Marathi (Ma), Hindi (Hi), Farsi (Far), Pashto (Pas), Mandarin (Man), Cantonese (Can), Inuktitut (Inu), Japanese (Jap), Korean (Kor), Arabic (Ar) Hebrew (Heb), Hungarian (Hu), Khanty (Kh), Estonian (Est), Udmurt (Ud), Yukaghir (Yu), Even (Ev), Evenki (Ek), Yakut (Ya), Turkish (Tur), Buryat (Bur), Central Basque (cB), Western Basque (wB), Wolof (Wo).

1.2.3. The Altaic and Ural-Altaic hypothetical families. We consider also the case of the Ural languages and the more hypothetical Altaic grouping. This is interesting because of the contested Ural-Altaic family hypothesis. In particular, while earlier attempts at identifying a hypothetical Altaic family had suggested the inclusion of both Korean and Japanese into this putative family, later studies had discarded the idea that these should be included, while it retained the possibility of an Altaic family encompassing languages like Turkish, Buryat, Yakut, Even, Evenki, Karachay, and Tatar. The inclusion of the Turkic languages like Tuvan and Uyghur in a hypothetical Altaic family is now also generally discarded. It was also hypothesized that both the Uralic and the Altaic languages should fit into a large Ural-Altaic family, although this hypothesis is also considered controversial, see [26], [40] for an overview. The LanGeLin syntactic data have already been used recently to study these families and the Ural-Altaic hypothesis, [21. We discuss here what information one can extract about this historical linguistic hypothesis from the persistent topology method.

The SSWL database has few languages that can be used to investigate the Ural family and the Altaic and Ural-Altaic hypothesis:

- Uralic: Estonian, Finnish, Hungarian, Udmurt.

- Altaic: Karachay, Tatar, Turkish, Tuvan, Uyghur.

- Previously hypothesized as Altaic: Japanese, Korean, Okinawan.

The LanGeLin data have as list of languages covered by the hypothetical Ural-Altaic classification:

- Uralic: Estonian, Finnish, Hungarian, Udmurt, Yukaghir, Khanty.

- Altaic: Turkish, Buryat, Yakut, Even, Evenki.

- Previously hypothesized as Altaic: Japanese, Korean. 
1.2.4. Comparative performance of datasets. The comparative analysis carried out in [31] shows that the SSWL and the LanGeLin datasets behave very differently in terms of clustering properties. This finding will be confirmed, by different methods, in the present paper. The results of [37] show that both dataset perform reasonably well in terms of phylogenetic reconstruction, provided some care is taken into dealing with the lacunae of the SSWL data, but still with a tendency for the LanGeLin dataset to have better performance. Again we will confirm here, with a different method, that the LanGeLin data behave better in terms of phylogenetic reconstruction, although the trees we construct in this paper should not be regarded as phylogenetic trees but as hierarchical clustering structures of syntactic features.

\section{Cluster analysis}

The first thing to observe when looking at the number of clusters by radius (see Figure 4 , Figure 5 and Figure 6) is that different language families (Indo-European, Austronesian, Niger-Congo, and Afro-Asiatic) exhibit different cluster structures. The Indo-European family consists of the largest number of non-trivial clusters (Figure 4). Other language families (see Figures 4 and 5) barely have non-trivial clusters at any given radius. This might suggest, as mentioned in [35], that in the case of language families with a smaller amount of non-trivial clustering the syntactic parameters are more centered and homogeneously distributed across the different languages compared to the Indo-European family.

The Longobardi LanGeLin data set contains mainly Indo-European languages so we can compare the cluster analysis of the LanGeLin data in Figure 6 to the clustering of the Indo-European language family in the SSWL database, Figure 4.

2.1. Singletons and clusters. Another interesting point is that in the SSWL there are singletons (i.e clusters containing only one data point) for every radius, and in every language family (except the Austronesian). In the Longobardi LanGeLin data, on the other hand, starting from a certain radius there are no more singletons. Linguistic interpretations for this phenomena may involve the presence in the SSWL database of languages that are farther away from each other, or the different nature of the SSWL syntactic features.

In fact the different clustering structure of the SSWL and the LanGeLin data appears to reflect the different nature of the syntactic variables that are recorded in the two databases. Indeed, in the analysis carried out in [31, where the data are analyzed by syntactic parameters rather than by languages, a similar phenomenon is observed, whereby the SSWL data contain more singletons that last of a wider range of scales before being incorporated into clusters, while in the LanGeLin data clusters form more rapidly. Since the analysis of [31] is by syntactic features across languages rather than by languages, no difference is detected there between different language families, while in the cluster analysis we performed here we see a clear difference between different language families.

One can raise the question of whether a part of this effect may also be due to the inherent problems of the SSWL data, namely the fact that languages are not uniformly mapped. However, this would more likely create an opposite effect, where languages with a large set of incomplete binary variables may appear closer than they really are because of a large overlapping set of lacunae. Similarly homeoplasy effects due to languages that are historically far away from each other being closer at the level of syntactic structures would also tend to produce an opposite effect with more rather than less clustering. 


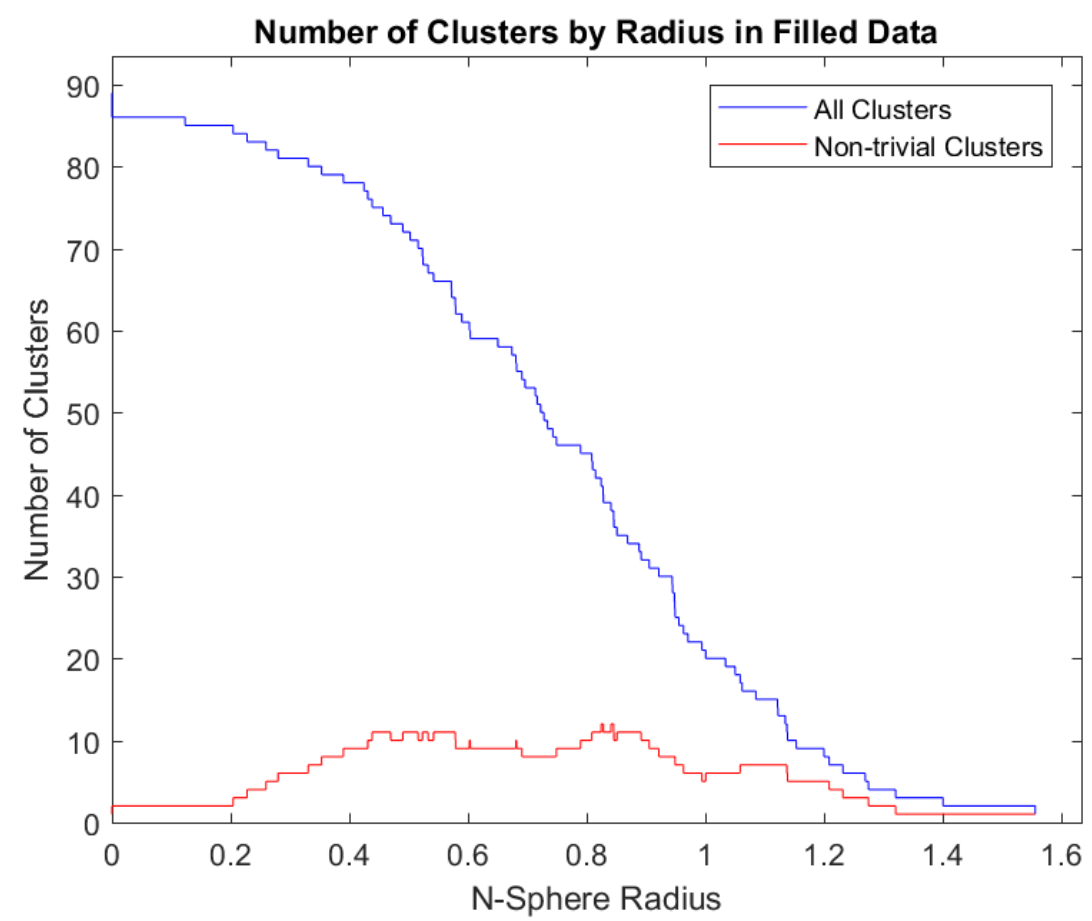

(A) Indo-European

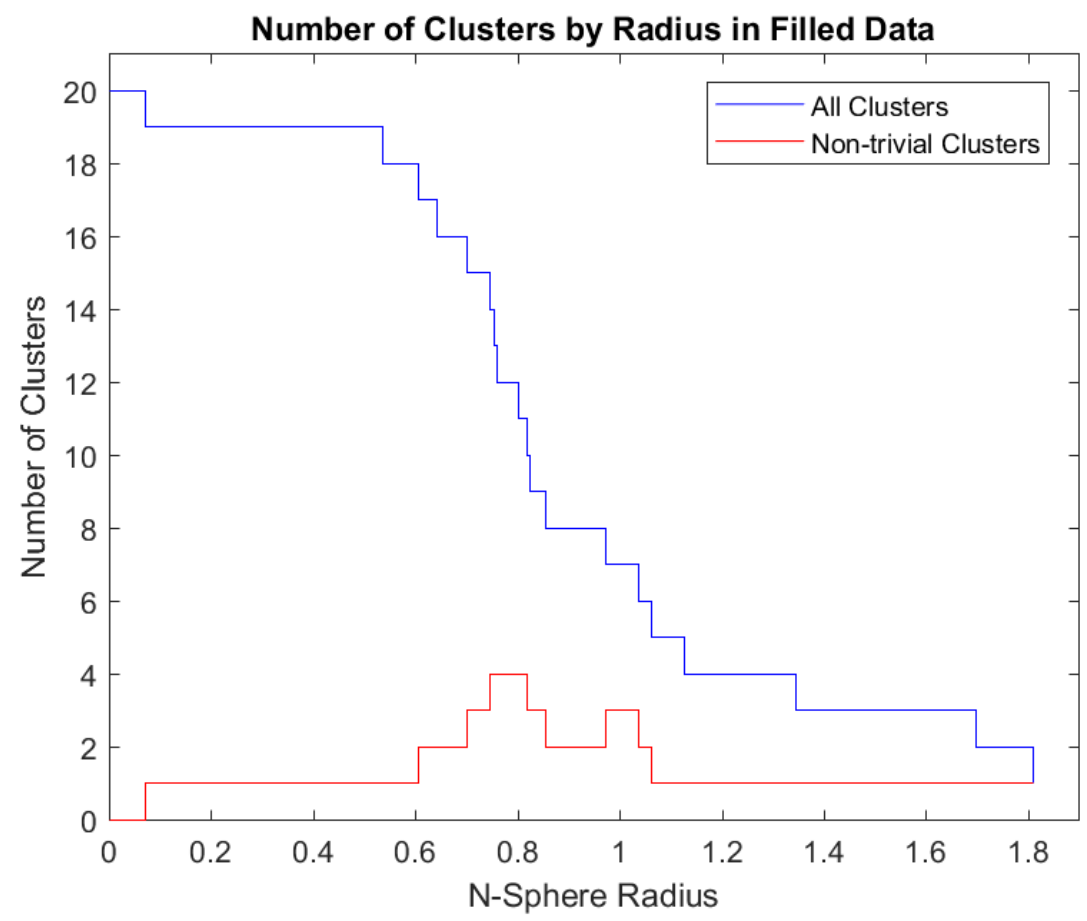

(B) Austronesian

Figure 4. Number of clusters by radius for the SSWL data for the IndoEuropean and Austronesian languages, with PCA percent variance $60 \%$. 


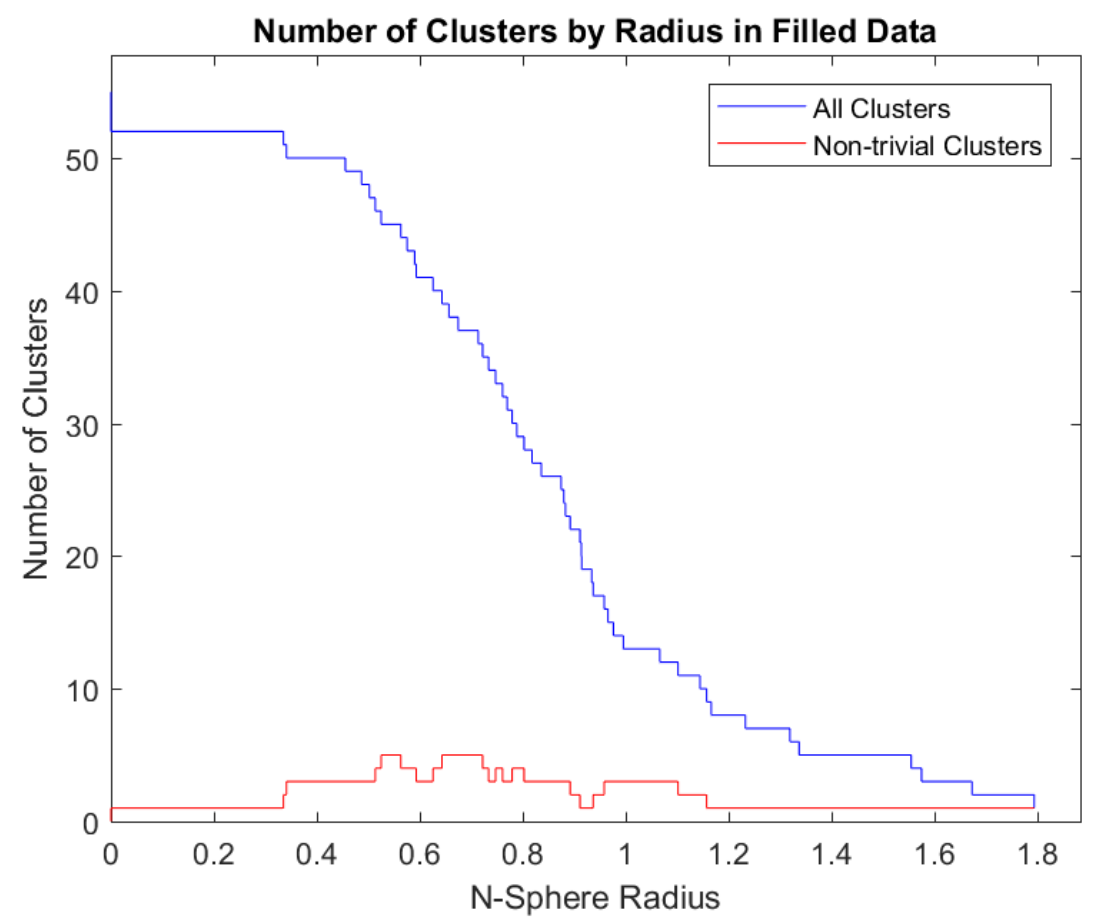

(A) Niger-Congo

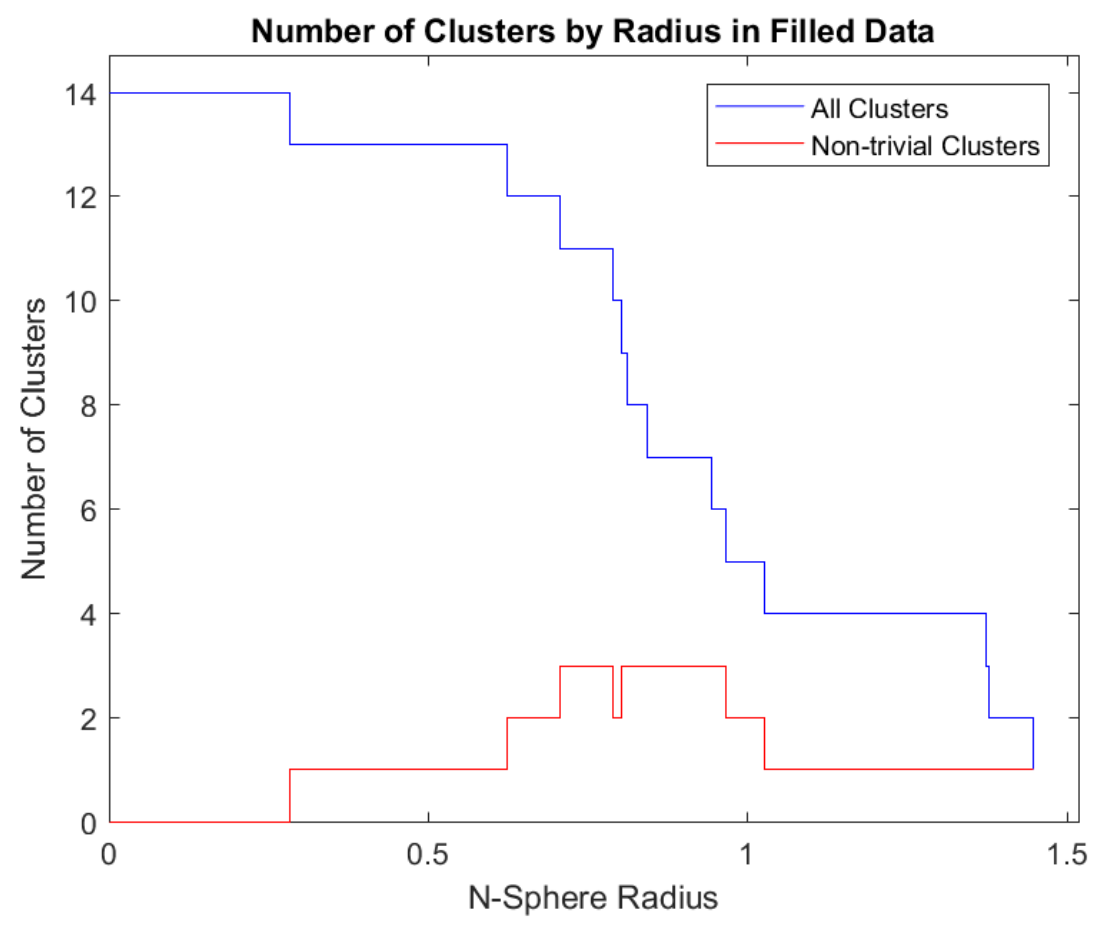

(B) Afro-Asiatic

FIgURE 5. Number of clusters by radius for the SSWL data set for the Niger-Congo and Afro-Asiatic languages, with PCA percent variance $60 \%$. 


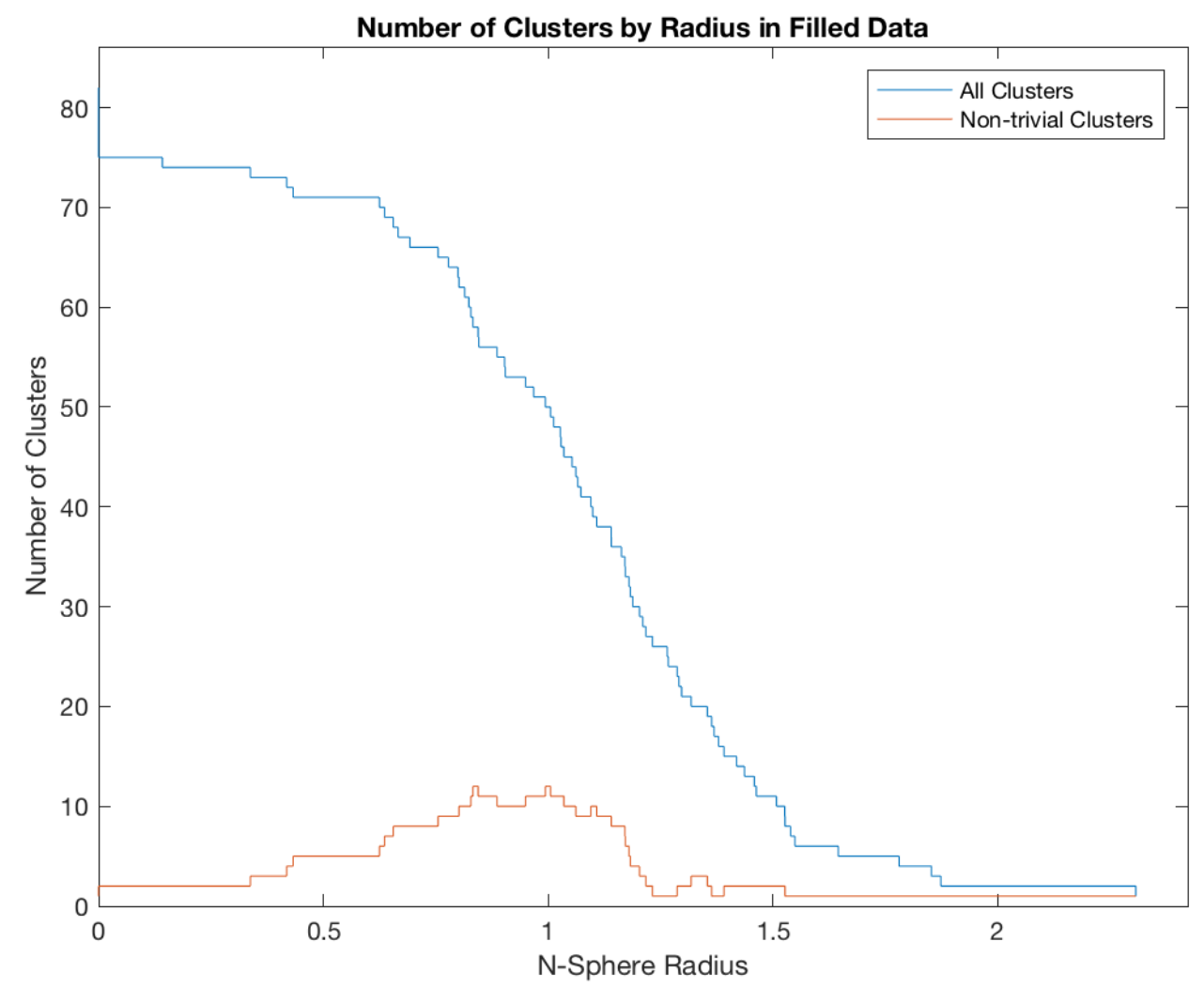

Figure 6. The number of clusters by radius for the LanGeLin data set, with PCA variance $60 \%$.

2.2. Clustering and the role of Greek-Italian Microvariations. In [13], an in depth study of linguistic diversity is carried out for a range of Romance and Greek dialects, analyzed at the syntactic level using the LanGeLin data of syntactic parameter. This type of analysis of "microvariations" applies to languages that are either genealogically very closely related or belong to distinct genealogical groups but are in close proximity and interaction within a limited geographic region. This is indeed the case of a range of dialects between the Romance and Greek language families in the Southern region of Italy. The languages in the LanGeLin database that are involved in this microvariations structure are the Romance dialects Ragusa (RGS), Mussomeli (MuS), Aidone (AdS), Northern Calabrese (NCa), Southern Calabrese (SCa), Salentino (Sal), Campano (Cam), and in the Greek family the dialects Salento Greek (SaG), Calabrian Greek A (CGA), and Calabrian Greek $\mathrm{B}$ (CGB).

We observe here, by looking at the position of the Greek languages within the two databases, that the very different clustering structure they exhibit with respect of the rest of the Indo-European languages may reflect the fact that the LanGeLin data include all the information about these microvariations in the region between the Romance and Greek languages, while the SSWL does not. 
More precisely, a more in depth look into the clusterification of the two data sets, via the tree that keeps track of how singletons merge into non-trivial clusters as the scale parameter increases, reveals an interesting difference with respect to the role of the Greek language family. In the SSWL dataset, the farthest/last singletons that are added are Cappadocian Greek, Ancient Greek and Greek. Then, other Greek languages occur, Medieval Greek and Homeric Greek, along with other languages such as Kurdish, Latin, and late Latin. Only then we get the first non-singleton clusters. In other words, in the SSWL data for the Indo-

European languages, the Greek languages are added later as farthest from the rest of the languages in the data set and far from each other. The only Greek languages that are not added later are highly incomplete languages, hence we can ascribe their faster clustering to a spurious effect due to lacunae. All of the Greek languages mentioned above are highly completed with more than $85 \%$ parameters filled in most of them, and not less than $65 \%$ parameters filled in the rest, so their singleton behavior can be considered reliable and not affected by incompleteness.

In the Longobardi data set, on the other hand, the Greek languages have neighbors quite early on and are not isolated for long. This in part reflects the presence in the LanGeLin database of the set of Southern Italian dialects corresponding to the GreekItalian microvariation, with the dialects Salento Greek, Calabrian Greek A, and Calabrian Greek B belonging to the Greek family. As we will see when we discuss the persistent components tree for the LanGeLin data, we also find a proximity between Bulgarian and some of the Greek languages, see Section 6.3.3.

\section{Principal Components and Persistent Topology Barcodes}

For the sake of computation time and in order to investigate the relations between the parameters, we introduce PCA as a method of dimensional reduction on the data. PCA rewrites the data in an ordered way that lets us reduce its dimension based on variance. We observe that running the analysis at different levels of PCA variance preserved (e.g. $60 \%$ and $80 \%$ ) does affect the structure of the clusters (hence the structure of the resulting persistent components trees). However it does not do so in a major way. This means that typically the varying the PCA variance level can cause some displacements of branches within smaller subfamilies, but does not affect the major subdivisions into main clusters. We will illustrate in detail an example where one can see an effect caused by varying the level of PCA variance preserved, when we discuss the case of the Germanic languages in Section 6.4.1. Since some form of PCA is often used in the phylogenetic reconstruction based on syntactic parameters, it is useful to keep in mind that there can be effects due to the level of PCA variance that can slightly alter the form of the resulting trees.

Our data are originally recorded as a discrete data set but after PCA it is given by continuous variables and the distance between the points is given by the Euclidean distance in the ambient space. Based on these data of distances, the Perseus program [43] builds a simplicial complex at each radius and computes the persistent homology. The outputs are given as barcode graphs and 1-skeleta as illustrated in Figure 7, $2 D$ and $3 D$ scatters of the data in the first 2 and 3 principal components, as shown in Figure 8 , and the number of clusters in each radius, as in Figure 7 and Figure 6. A more thorough explanation of the this part of the code can be found in [35. 

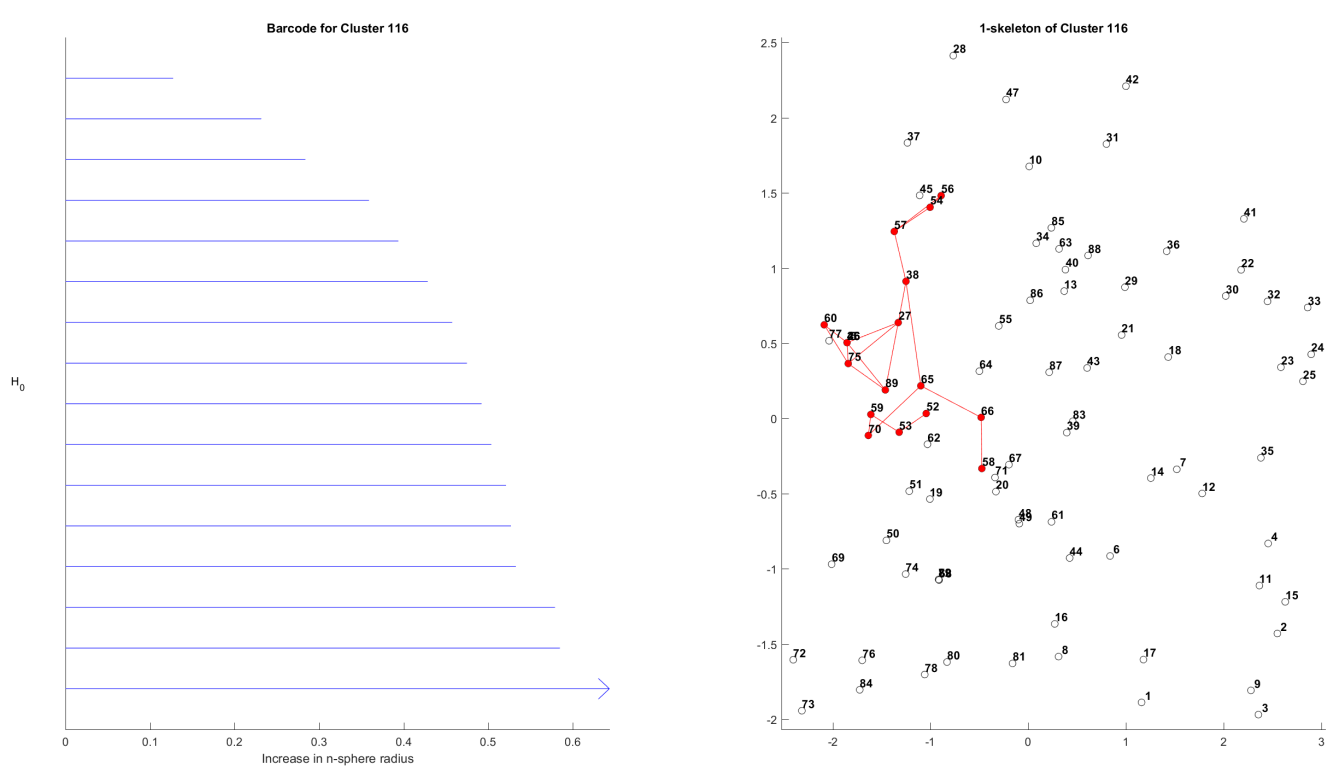

(A) filter value 116
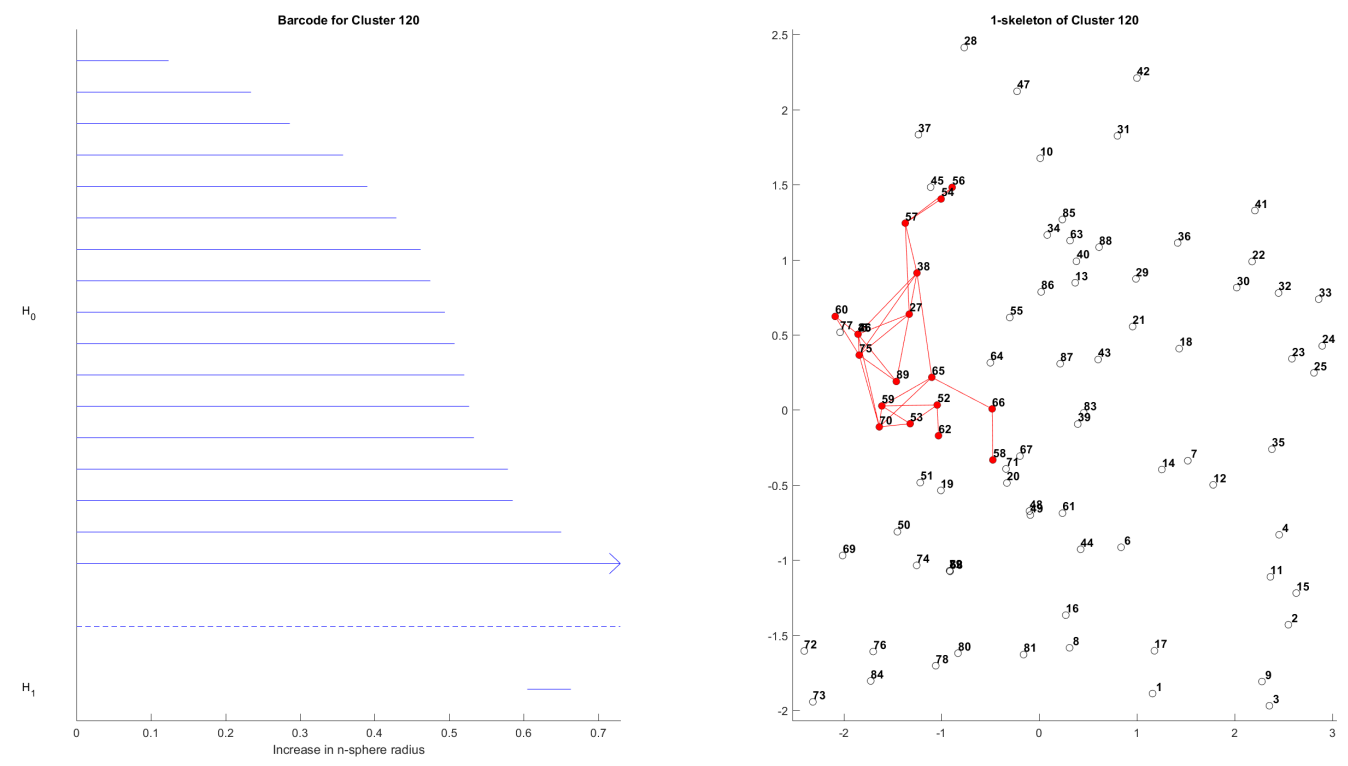

(B) filter value 120

FiguRE 7. Barcode graphs and their corresponding 1-skeleta at filter values cluster 116 and cluster 120, SSWL data. 
The use of a dimensional reduction method like PCA prior to the persistent homology computation performed by Perseus is necessary in order to make the problem computationally tractable, as Perseus would not be able to handle the persistent homology computation over the entire high-dimensional space.

A comment that will be useful later, when we analyze the persistent components trees of various subfamilies within larger families, is the construction of the radius and steps in radius increase in our algorithm. We start, for a given set of data, by computing a critical radius $r_{c}$ which is the minimal length such that by drawing balls of radius $r_{c}$ around each point in the set one obtains a single connected component. Then a scale $\epsilon$ is fixed to be a chosen fraction of that radius, such as $\epsilon=r_{c} / 100$, and the algorithm then increases the radius in steps of $\epsilon, 2 \epsilon, \ldots, N \epsilon \ldots$. It is possible that, if the initial critical value is computed over the entire dataset, when restricting to subfamilies of very closely related languages, the chosen step size $\epsilon$ will not be large enough to resolve all the points in the subfamily, missing some of the tree structure. It is then necessary to adapt the radius $r_{c}$ and the size steps $\epsilon$ to the individual subfamilies to resolve all the languages in the subfamilies.

3.1. Principal components and weighted syntactic structures. The PCA method, as mentioned above, replaces the original set of binary variables, seen as vectors with binary coordinates inside an ambient real Euclidean space, with a new set of real variables given by the components of the original vectors along a new basis given by the principal components.

In other words, we can describe the principal components as a weighted sum of the original coordinate axes that provide the new set of coordinate axes that maximize variance so as to replace a set of possibly correlated variables by a set of values of linearly uncorrelated variables.

In our setting the original coordinate axes are identified with the different binary syntactic variables (syntactic parameters), while the principal components are then weighted sums of these original variables. Thus, the process of computing principal components can be viewed as an assignment, based on the data of the set of languages considers, of a real valued weight to each syntactic parameter/variable. The process reduces the dimensionality of the data by replacing the full set of original variables by the first few principal components, see Figure 8 .

Since the principal components select a set of new linearly independent variables, this weight assignment can be interpreted heuristically as a measure that weights more heavily those syntactic parameters/variables that are more likely to be genuinely independent variables while weighting less those that are more dependent on the others. Thus, linguistically we can think of this operation as replacing the original choice of the syntactic parameters by a new weighted system of the same variables which express the coordinates of the new principal components.

It was argued in [37] and [41 that for the purpose of considering dynamical models of language change, either in the Markov models on trees used in the phylogenetic algebraic geometry method in [37] or in the spin glass models considered in [41] one should not consider it satisfactory to assume that the syntactic parameters are independent identically distributed random variables, because of the existence of unspecified relations between them. Thus, it would be more natural to weight the syntactic parameters by an indicator of dependence/independence. One natural choice of how to do that is by using as weights the coefficients of the principal components that maximizes variance. 


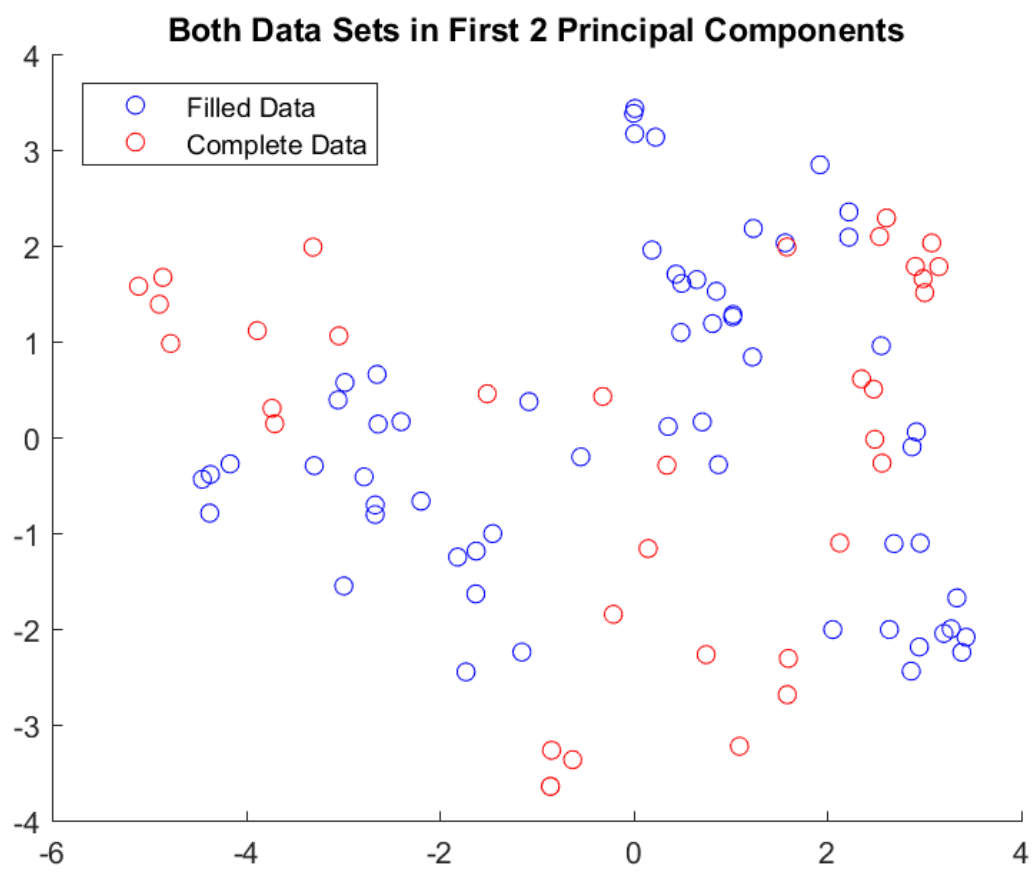

(A) $2 D$ scatter

Both Data Sets in First 3 Principal Components

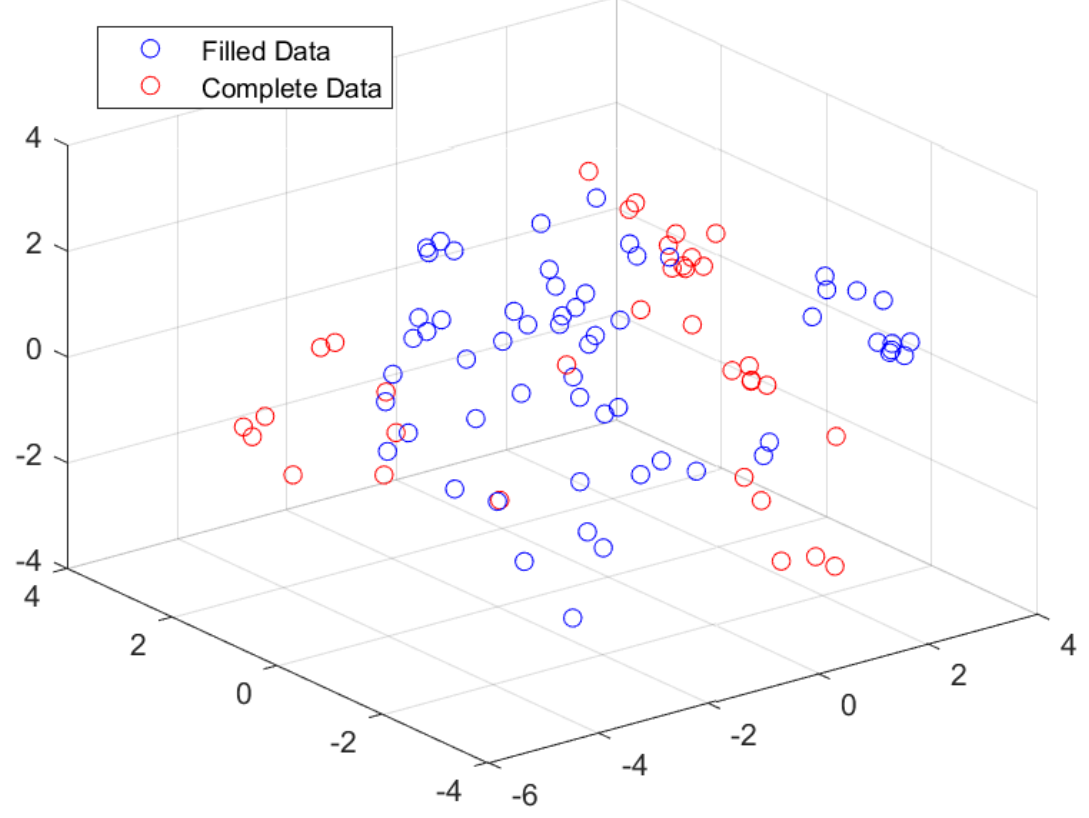

(в) $3 D$ scatter

Figure 8. $2 D$ and $3 D$ scatters of the data in the first 2 and 3 principal components. 
We observe that the principal components look quite different in the case of the SSWL data and in the case of the LanGeLin data. The different structure indicates fewer linear relations among the SSWL variables than among the LanGeLin variables. Notice though that the absence of linear relations does not indicate an absence of relations of other kinds, see for instance the results of [33] for a strong indication of other relations among the SSWL variables.

In the next section we discuss more in detail the question of the dimensionality of the data of syntactic structures, as a way to better detect the presence of relations between the syntactic variables, beyond what can be seen in terms of principal components.

\section{Dimensionality analysis}

One of the main problems when analyzing syntactic data is the fact that the parameters are not at all independent variables. Investigating the relations between the parameters and finding a good set of "universal coordinates" for the space of syntactic parameters, or in other words, finding "the geometry of syntax" can be regarded as one of the main open questions in Chomski's Principles and Parameters model of syntax. Therefore, we would like to estimate the dimension of the data in order to get a better sense of the dependencies and the relations between the parameters.

\subsection{Description of the algorithm.}

- Let $D \subseteq \mathbb{R}^{d}$ be our data set. We choose $p \in\{1, \ldots,|D|\}$ and a point $\vec{x}_{1}^{(p)}$. We then sort $D$ into a vector $\left\{\vec{x}_{i}^{(p)}\right\}_{i=1}^{|D|}$ where the entries are ordered by distance from the selected point, i.e. where the distances monotonically increase,

$$
d\left(\vec{x}_{i}^{(p)}, \vec{x}_{k}^{(p)}\right) \leq d\left(\vec{x}_{j}^{(p)}, \vec{x}_{k}^{(p)}\right) \quad \text { for } i \leq j .
$$

- Choose $s \in\{1, \ldots,|D|\}$ to be the number of nearest neighbors and define

$$
X^{(p, s)}=\frac{1}{d\left(\vec{x}_{s+1}^{p},{\overrightarrow{x_{1}}}^{p}\right)}\left[\begin{array}{c}
\vec{x}_{2}^{(p)}-{\overrightarrow{x_{1}}}_{1}^{(p)} \\
\vec{x}_{3}^{(p)}-\vec{x}_{1}^{(p)} \\
\vdots \\
\vec{x}_{s+1}^{(p)}-\vec{x}_{1}^{(p)}
\end{array}\right] .
$$

- The data is spread out and we shift the selected points and their $s$-nearest neighbors so that they will fit into the $d$-dimensional unit ball centered at the selected point.

- Let $W^{(p, s)} \in M_{s \times s(\mathbb{R})}$ be the weight matrix with the following properties:

(1) $W^{(p, s)}$ is diagonal,

(2) $\operatorname{det}\left(W^{(p, s)}\right)=1$,

(3) $W_{i, i}^{(p, s)} \sim e^{\frac{-d\left(\vec{x}_{i+1}^{(p)}-\vec{x}_{1}^{(p)}\right)^{2}}{\alpha}}$.

- The range where $\alpha$ is small corresponds to the local behavior and the range where $\alpha$ is large to the global behavior.

- Following a common best fit algorithm we now define a weighted covariant matrix:

$$
C^{(p, s)}=\frac{1}{s}\left(\vec{x}^{(p, s)}\right)^{T} W^{(p, s)} \vec{x}^{(p, s)},
$$



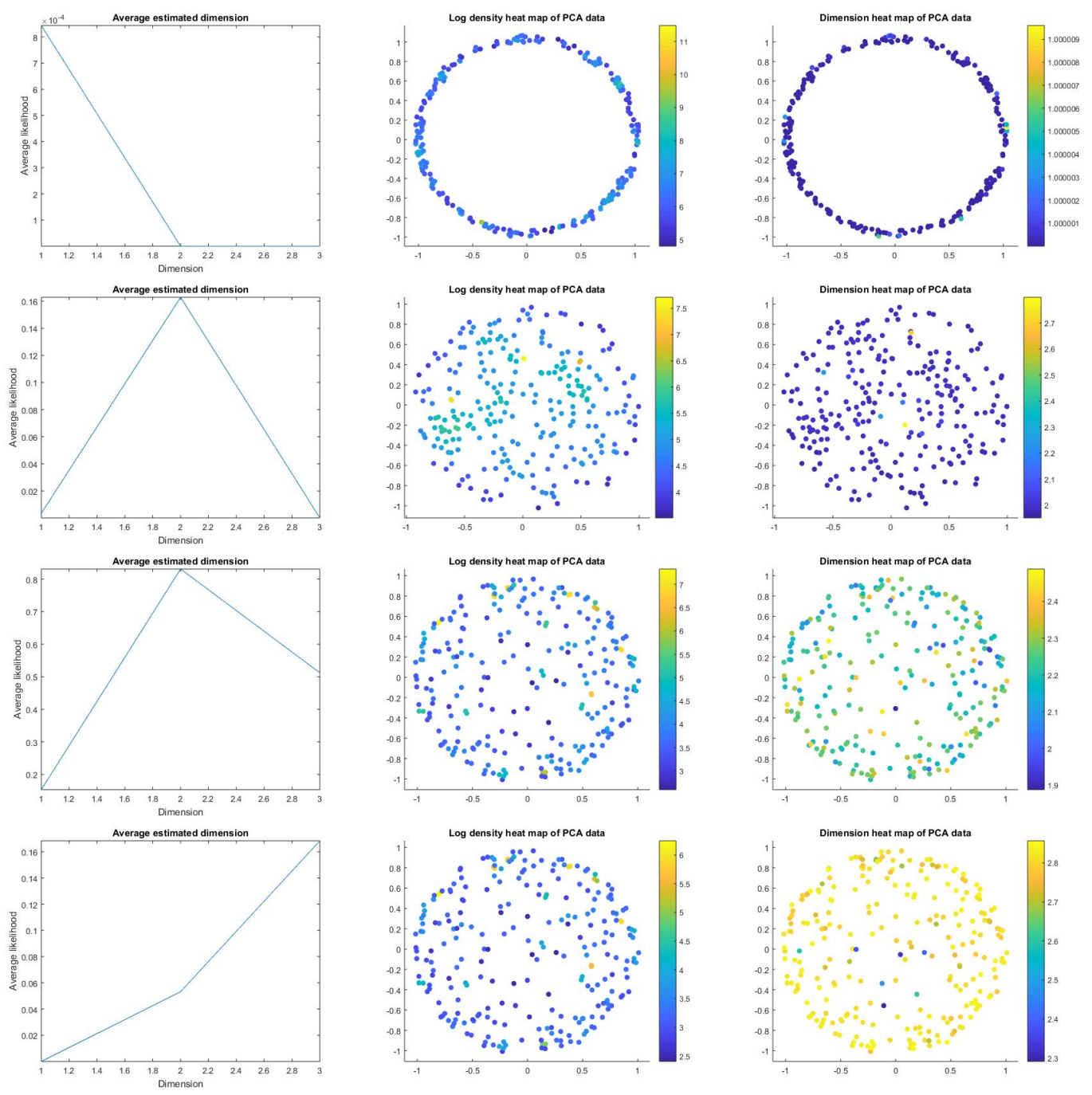

FiguRE 9. Dimensionality for simple geometries: 1-sphere with $\alpha=1 / 3$, 2-ball with $\alpha=1 / 3$, and 2-sphere with $\alpha=1 / 10$ and $\alpha=1 / 3$ in $\mathbb{R}^{3}$.

- We compute its eigenvalues $\lambda_{1}^{(p, s)} \geq \cdots \geq \lambda_{d}^{(p, s)}$ and corresponding eigenvectors $\left\{\vec{v}_{\lambda_{1}}^{(p, s)}, \ldots, \vec{v}_{\lambda_{d}}^{(p, s)}\right\}$ in the same ordering. The output is the eigenbasis matrix $V^{(p, s)} \in$ $M_{d \times d}(\mathbb{R})$ where the $i$-th column corresponds to $\vec{v}_{\lambda_{i}}^{(p, s)}$.

- We choose $f \in\{1, \ldots, s\}$, the dimension of fit. Let $P^{(f)} \in M_{d \times d}(\mathbb{R})$ such that $P^{(f)}$ is diagonal and

$$
P_{i i}^{(f)}=\left\{\begin{array}{l}
0, \text { if } i \leq f \\
1, \text { if } i>f
\end{array} .\right.
$$

- The $(p, s, f)$-error is given by:

$$
W^{(p, s)} X^{(p, s)}\left(V^{(p, s)^{T}}\right)^{-1} P^{(f)} V^{(p, s)^{T}},
$$


where the magnitude of the $i$-th row of $X^{(p, s)}\left(V^{(p, s)^{T}}\right)^{-1} P^{(f)} V^{(p, s)^{T}}$ is the orthonormal error of the $i$-th nearest neighbor.

- Compute all the $(p, s, f)$-errors on the selected data and also on balls and spheres of $\operatorname{dim} \leq d$ for comparison.

- Run paired $T$-test between the selected points and balls/spheres database to get maximum likelihood $T$-value of $T$, see Figure 11 .

4.2. Density estimation. For our data set $D \subset \mathbb{R}^{d}$ we define $R^{(p)} \in M_{(|D|-1) \times 1}$ as

$$
R_{i}^{(p)}=\frac{1}{\operatorname{Vol}\left(B^{d}\right) \cdot d \cdot d\left(\vec{x}_{i+1}^{(p)},\left(\vec{x}_{1}^{(p)}\right)^{d}\right.} .
$$

Computing then the quantity

$$
\frac{\sum_{i} W_{i, i}^{(p,|D|-1)} R_{i}^{(p)}}{\sum_{i} W_{i, i}^{(p,|D|-1)}}
$$

for every $p$ gives us a heat density map, see Figure 12

4.3. The test case of spheres and balls. When running the algorithms described above for simple geometries like the circle, the disk, and the 2 -sphere, we see more clearly the role of the $\alpha$ parameter and the information contained in the heat-map. In these cases the dimension is clearly identified, although for larger values of $\alpha$ the dimension counting can appear altered towards the larger dimension of the ambient space rather than the actual dimension of the submanifold. In the heat-map, while random points on the sphere have a more homogeneously distribution of densities, in a ball or disk those points located near the boundary tend to show a higher density than points located deeper into the bulk, see Figure 9 .

4.4. Estimated dimension of the space of syntactic structures. In this dimensional analysis we have been considering the transposed data matrix, where each data point corresponds to a syntactic parameter/syntactic variable and the vector entries correspond to a language with the value of the parameter for that language recorded. This means that we are looking at how syntactic features are distributed across languages and we would like to identify the dimension of a submanifold of the full ambient space on which the data points lie.

After running the code implementing the algorithm described above on both the LanGeLin data set and the SSWL data set for various $\alpha$ values, we observe the following outcomes.

For the LanGeLin data set at $\alpha=\frac{1}{3}$ (see Figure 11 and Figure 12 we obtain that the estimated dimension of the data is $d \sim 15$, whereas for $\alpha=0.1$ it is too noisy to approximate, see Figure 11. For the SSWL data, on the other hand, we get that for $\alpha=1$ the estimated dimension of the space of syntactic variables peaks at around $d \sim 30$, see Figure 10. 

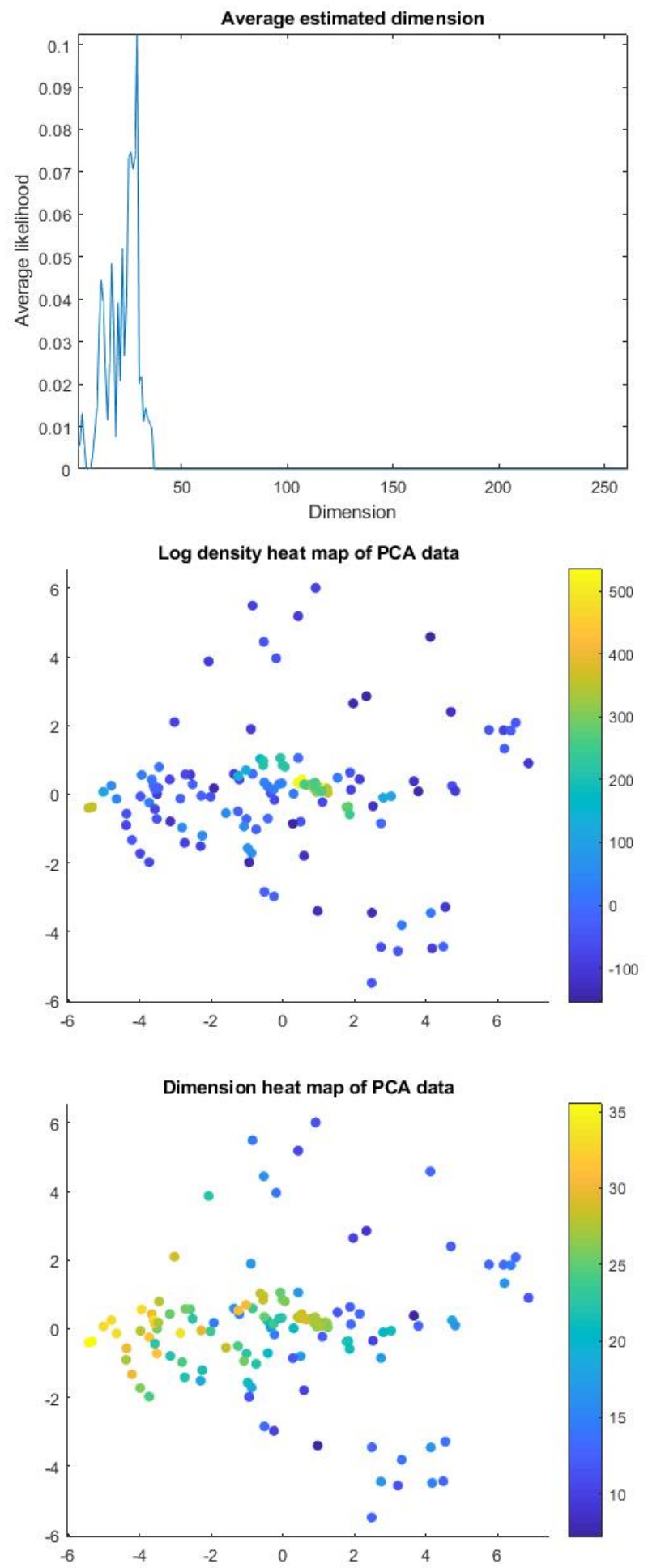

FIGURE 10. Dimensionality of the space of syntactic variables for the SSWL data at $\alpha=1$. 

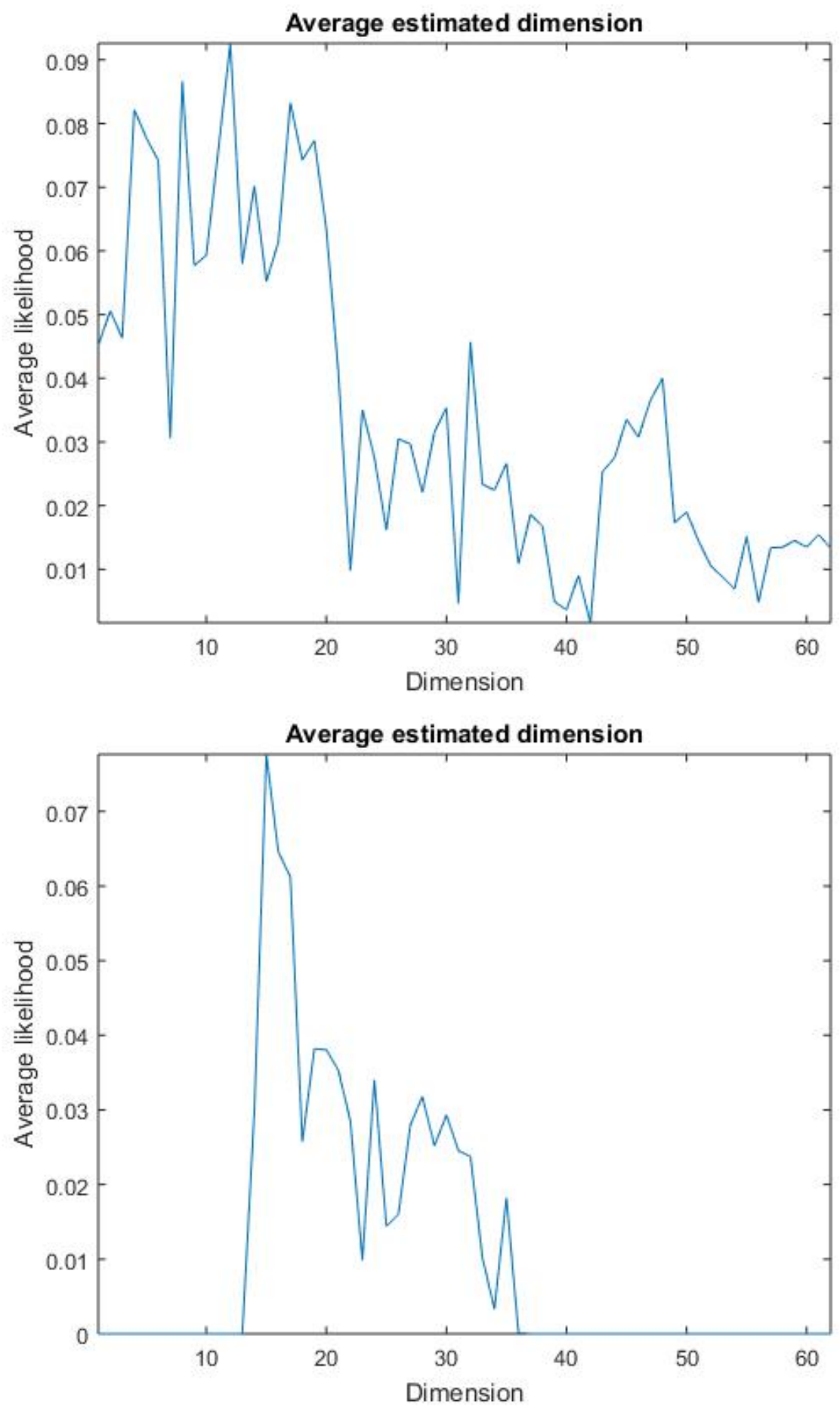

Figure 11. Dimension analysis of the LanGeLin data for $\alpha=0.1$ and $\alpha=\frac{1}{3}$ when averaging over the points. 

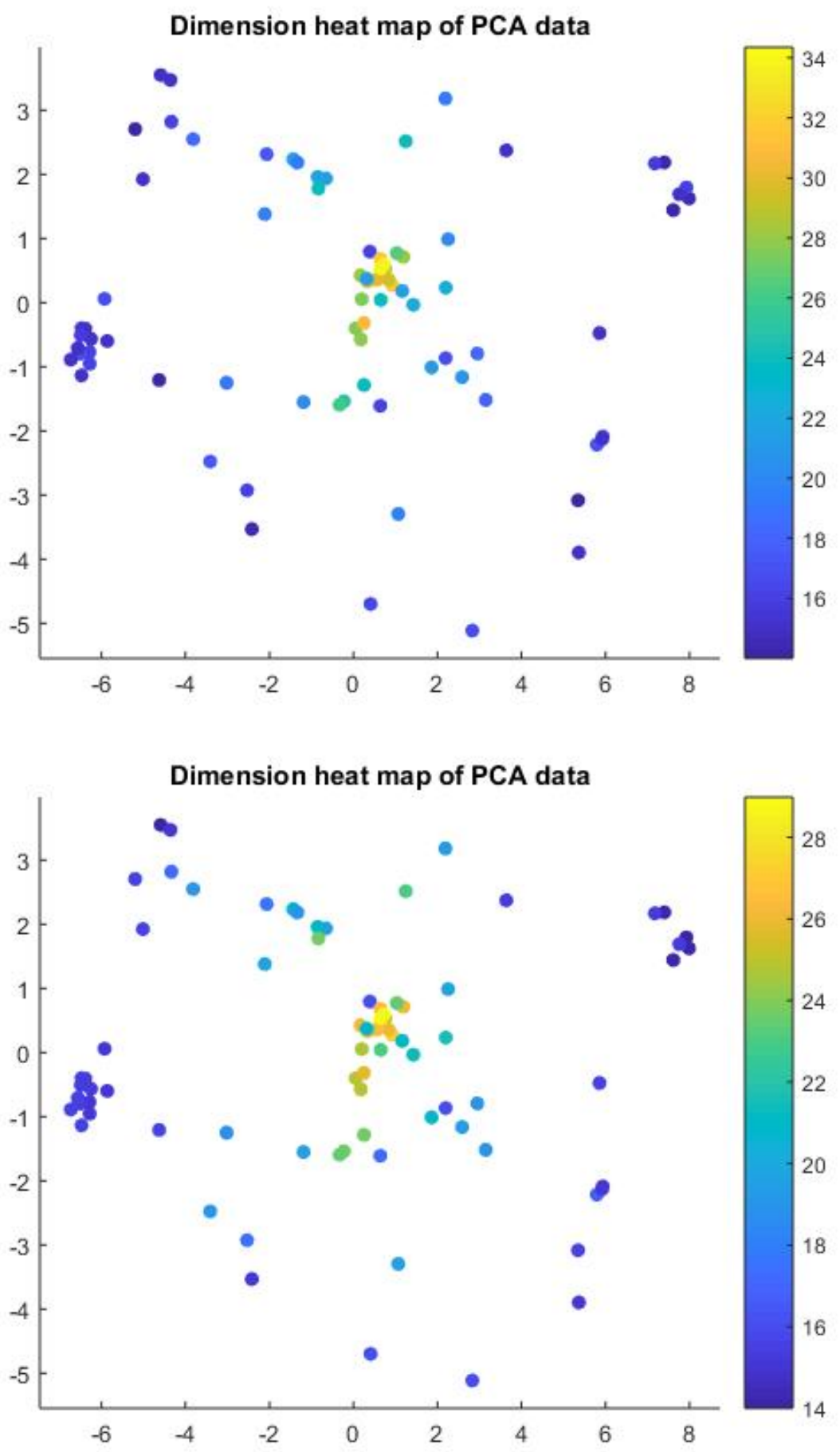

Figure 12. Dimension analysis of the LanGeLin data for $\alpha=0.1$ and $\alpha=\frac{1}{3}$ when averaging over the dimensions. 

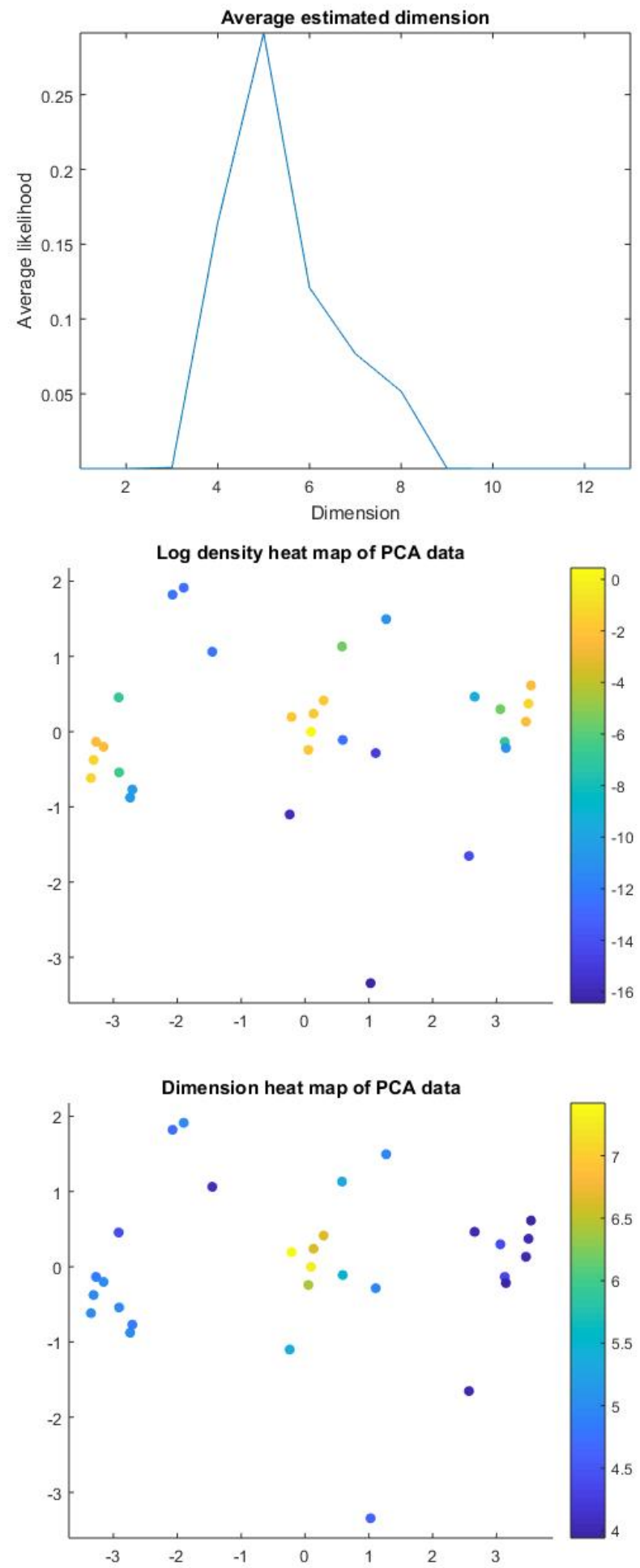

FiguRE 13. Dimensionality of the space of syntactic parameters for the Romance language family (LanGeLin data, $\alpha=1 / 3$ ). 

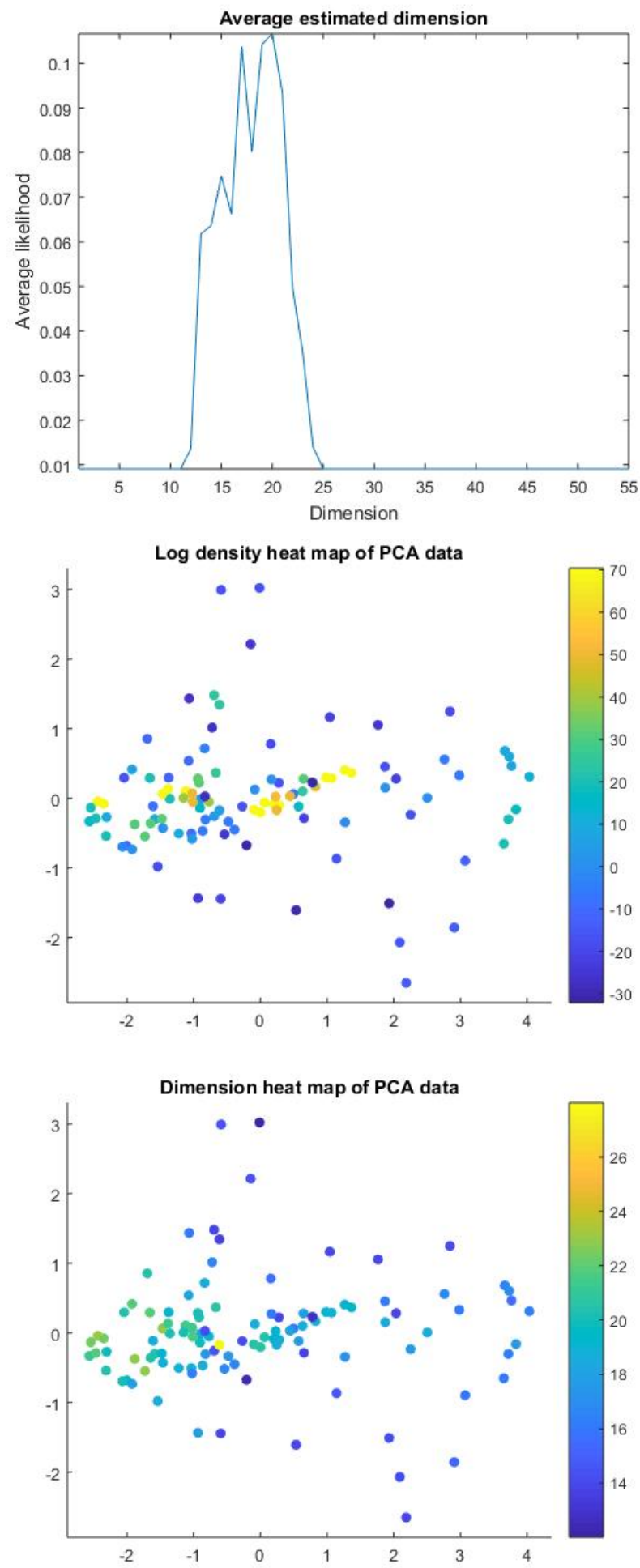

FiguRE 14. Dimensionality of the space of syntactic variables for the NigerCongo language family (SSWL data, $\alpha=1 / 3$ ). 

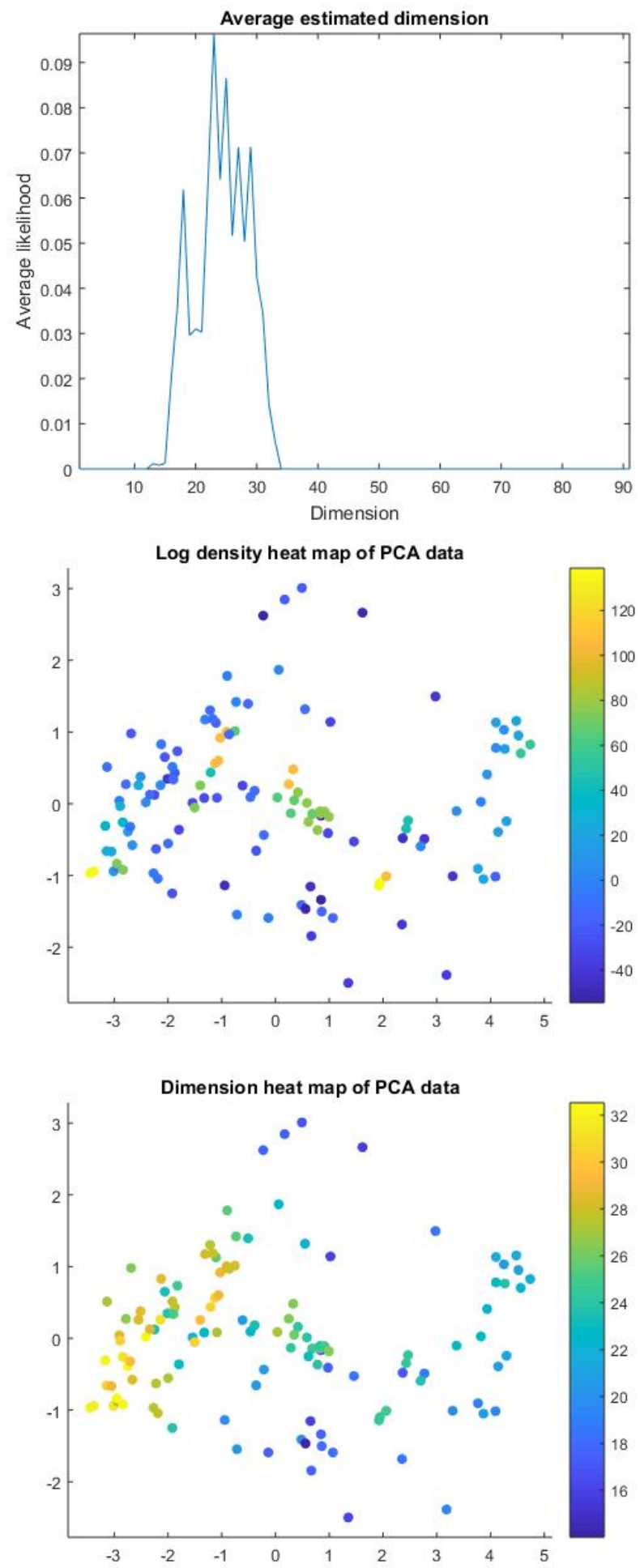

FiguRE 15. Dimensionality of the space of syntactic variables for the IndoEuropean language family (SSWL data, $\alpha=1 / 3$ ). 
4.5. Dimension estimates of syntactic structures within language families. One can also consider the question of whether there are relations between syntactic parameters that only hold within certain language families rather than being universal across languages. If this is the case, then we expect to find different dimension estimates for the space of syntactic structures when only the coordinates of a given syntactic parameter that correspond to languages in a given family are retained.

We consider the subset of the LanGeLin data for the Romance languages and we look at the dimensionality of the space of syntactic parameters restricted to the set of Romance languages, see Figure 13 . We find a peak at a much lower dimension, $d=5$, than for the full data set across all languages, which has a peak around $d=15$. This implies the presence of additional relations between the syntactic parameters that are language specific rather than universal across language families.

We see a similar phenomenon when we consider the SSWL data and we restrict the syntactic variables to certain language families. For example we find that the dimension of the space of syntactic variables for the Niger-Congo languages peaks at a lower value, around 20, than the dimension for the space of Indo-European languages, where the dimension estimate peaks around 23 (see Figure 14 and Figure 15), suggesting that there are slightly more language-family-specific relations between the syntactic variables for the Niger-Congo languages than for the Indo-European languages.

4.6. Density and dimension estimates of language families. In the data matrix where data points are languages and coordinates are syntactic parameters/variables, one can also run the estimates of density and dimension discussed above. In this case, rather than describing relation between syntactic variables that are either universal across language families or family-specific, the estimates give us information about language relatedness through information about how spread out (in the sense of density and dimension) languages within a given family are then regarded in terms of their syntactic structures.

Thus, we consider the data points of certain given language families and we run the same dimensionality analysis discussed above, see Figure 16. When looking at the dimension estimates for the different language sub-families at $\alpha=\frac{1}{3}$ we see that we usually get clear peaks for the dimension estimation (versus larger $\alpha$ values, or larger data sets). Regarding the density maps, the estimations are less sensitive to the alpha value than the dimension estimates.

Over the entire set of languages of the SSWL database we find a dimension estimate around 38. The Niger-Congo family has dimension peak around 23, while the IndoEuropean language families has dimension estimate that is closest to the overall estimate, also around 38. The Afro-Asiatic and Austronesian language families have estimated dimensions respectively around 8 and 12 .

For the LanGeLin data, the dimension estimate for the space of languages is illustrated in Figure 17. The estimated dimension for the full set of languages in the database peaks around 25.

4.7. The Altaic and Ural-Altaic hypothesis. We discuss here the Altaic and UralAltaic hypothesis in terms of the dimensional analysis for these language families. This perspective, although carried out with a different mathematical method, is similar to the point of view discussed in [21] in their analysis of the Ural-Altaic hypothesis through the LanGeLin data. We use the same LanGeLin data here and we compute the dimension 

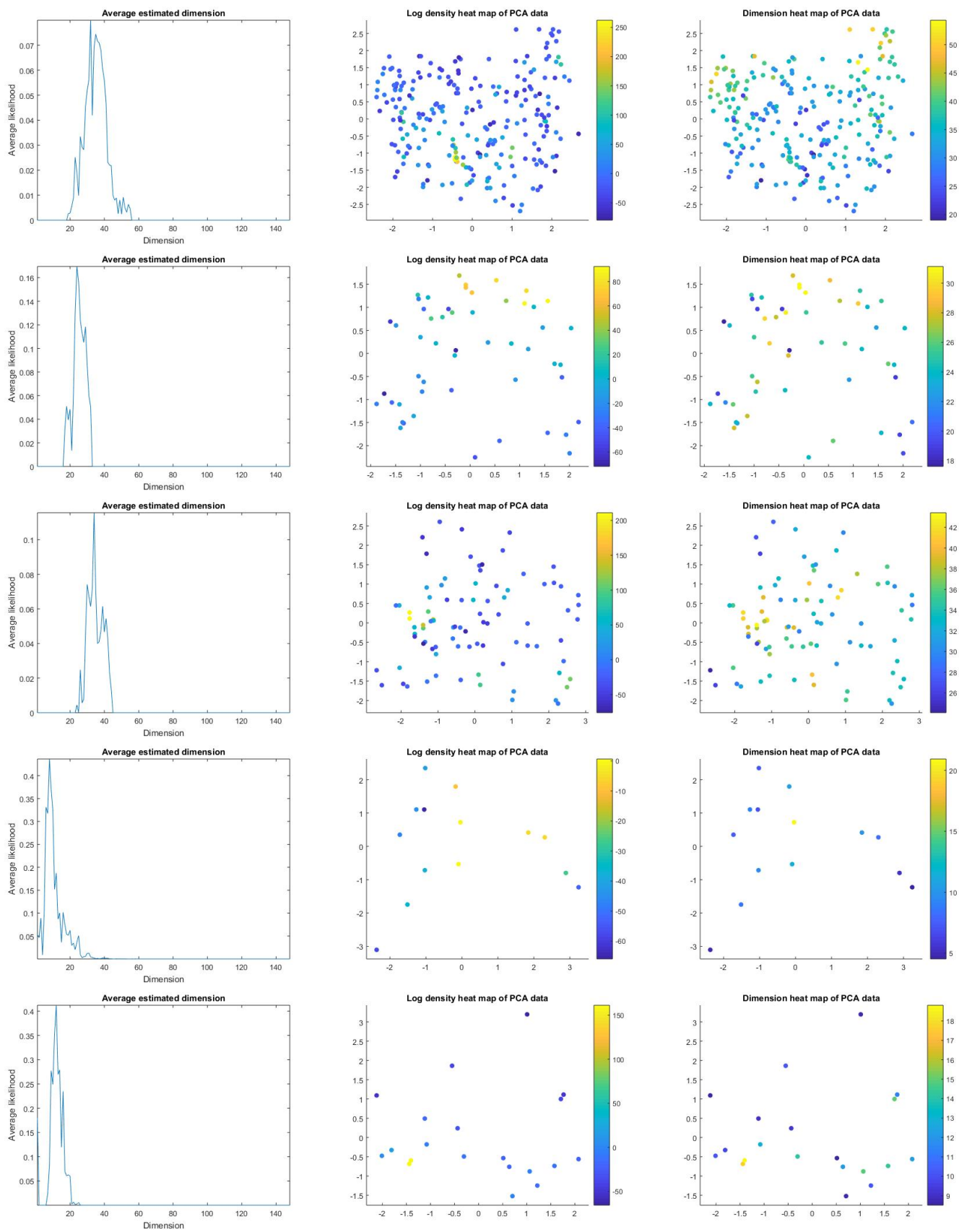

FiguRE 16. Dimensionality of the space of languages for the full database, the Niger-Congo family, the Indo-European family, the Afroasiatic family, and the Austronesian family (SSWL data, $\alpha=1 / 3$ ).

estimates separately for the Uralic and the Altaic languages and for the two sets together. The dimension distribution does not seem to rule out an Altaic hypothesis. In fact we find a clearer single peak for the estimated dimension for the Altaic languages than for the Uralic ones. However, when the Uralic and the Altaic families are joined together, the dimension 

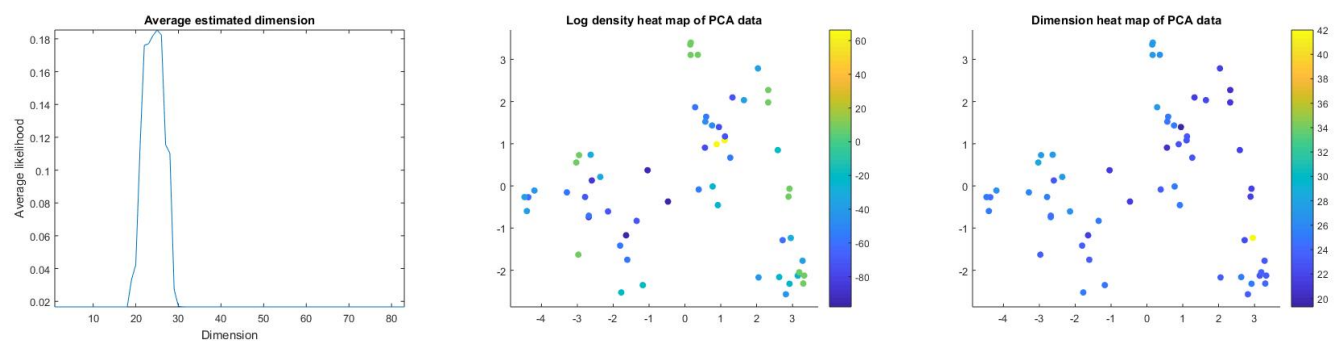

Figure 17. Dimensionality of the space of languages for the full database (LanGeLin data, $\alpha=1 / 3$ ).
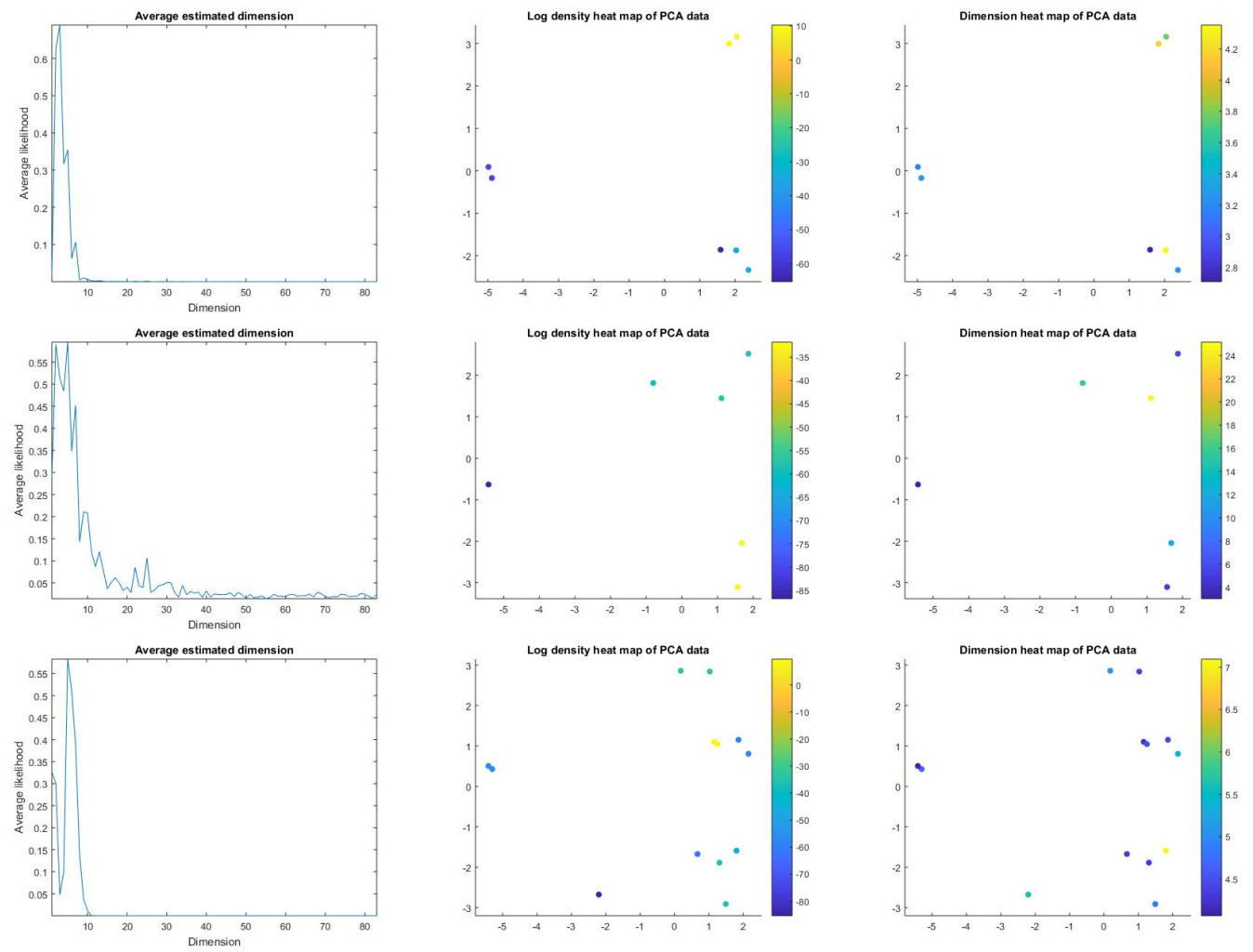

Figure 18. Dimensionality of the space of languages for the Altaic, Uralic, and Ural-Altaic languages (LanGeLin data, $\alpha=1 / 3$ ).

estimates shows two clear separate peaks. This may be interpreted as a cautionary indicator about the possibility of a single Ural-Altaic family hypothesis, see Figure 18.

\section{RELATiOns AND THE TOPOLOGY OF THE SPACE OF SyNTACTIC PARAMETERS}

In this section we return to the question of investigating relations between syntactic variables and identifying the topology and geometry of the space of syntactic parameters, previously discussed in [18, 31, 33], 38]. We have already discussed in Section 4 how the dimensionality analysis identifies a space of dimension around $d=12$ inside the space 
of the LanGeLin syntactic parameters, and a space of dimension around $d=30$ inside the 116-dimensional space of the SSWL syntactic variables, that represent the dimension of the submanifold on which the data of syntactic features are distributed. Thus, we know from these estimates that one should expect a high degree of dependence between the syntactic variables. As we have seen with dimensional analysis, there are additional family-specific relations that further lower the dimension when the data are restricted to specific language families. Here we seek to identify the relations between syntactic variables more explicitly by computing the persistent homology of the data set (organized with syntactic features as data points) and the tree of the persistent connected components.

When we consider the data points as syntactic features with coordinates giving the values of a feature over a set of languages and we compute the persistent homology of these data set, the clusters given by the $H_{0}$ identify subsets of syntactic features that are in close proximity to each other, in the sense that these syntactic features tend to be similarly expressed over subgroups of languages. It is clear that proximity between syntactic features detects some kind of relations between them (for example, if one is expressed in a language, so does the other). However, not all types of relations between syntactic parameters will be of this form: while persistent $H_{0}$ detects parameters that tend to align, it does not detect anti-alignments, nor it detects other forms of entailment such as those explicitly identified in the LanGeLin data. In this section we examine the proximity between the parameters using the phylogenetic tree built based on the $H_{0}$ computations and we compare briefly the resulting relations that we identify in this way to previous work aimed at identifying relations between syntactic features by different methods. In particular, we compare our results to the heat kernel analysis of [31] and to the analysis of the relations between the LanGeLin syntactic parameters via machine learning algorithms of [18].

5.1. Our tree construction algorithm. The main steps in reconstructing the persistent components trees are Principal Component Analysis (PCA), computation of the clusters and an inclusion-based tree reconstruction. Here is a step-by-step explanation of the Matlab script TreeFromDataGUI.m we wrote and used in this analysis.

- First the data is loaded and the files are labeled. Then, empty spaces in the data are filled with the midpoint of the data (empty spaces in the SSWL are filled with $\frac{1}{2}$ and in the Longobardi with 0 value). We also discuss trees obtained by filtering out languages in the SSWL that are too incomplete, see below.

- The second step is the PCA. It finds a PCA basis and takes up to the percent variance we choose (for our use it is either $60 \%$ or $80 \%$ ).

- Computes the Euclidean distance pairwise and finds the critical radius in which the simplicial complex becomes completely connected (i.e when we have one cluster). As noted previously, after the process of PCA our data becomes continuous, and therefore the metric we use is the Euclidean metric (rather than Hamming distance which is used in a discrete data set).

- Computes all the clusters in small incremental radii and assembles the persistent components tree based on inclusion. If $C_{r}$ is the set of all clusters at radius $r$ and $C=\sqcup_{r} C_{r}$ : in $C$, cluster $C_{i}$ is a child of cluster $C_{j}$ if $C_{i} \subseteq C_{j}$ and there is no cluster $C_{k}$ such that $C_{i} \subseteq C_{k} \subseteq C_{j}$.

In this section we will present a detailed analysis of persistent components trees of two different data sets, the SSWL data set and the LanGeLin data set, where we view the data 
points (in transpose form) as being the individual syntactic features and their coordinates being the values by languages. We use the resulting trees to identify structures of relations between the syntactic variables. The trees are constructed using the computational method described above, based on PCA and persistent homology computations, specifically the group $H_{0}$ that determines the persistent connected components of the data. We use the number on the vertex to refer to the same cluster number and also to the subtree that it forms.

5.2. Comparison with the heat kernel method. In [31] the BelkinNiyogi heat kernel dimensional reduction method [3] is used in order to analyze the relations between the syntactic parameters. This method gives more information than one gets by only using PCA, since it also gives connectivity relations (and not only proximity). Parameters that exhibit high connectivity to other parameters (with a higher valence in the resulting graph) are interpreted as more "dependent" and vice versa. The analysis of [31] is performed on both the LanGeLin and the SSWL data sets.

In our persistent topology analysis the LanGeLin data set has one main cluster which contains most of the points (N. 144), another small cluster (N. 156) and some singletons. Cluster 144 contains two subtrees (N. 122 and N. 153) and apart from that the other points are joined as singletons or 2-leaf trees, see Figure 19.

In the heat kernel analysis of [31, the Longobardi data set appears to have two main clusters (for scale size $\epsilon=15$ ). A big one composed by two sub-clusters consisting of the parameters EZ2, NOO, NOC, FGC, TPL, FGT, NSD, DPQ, GSI, HMP (the pink-colored points in Figure 19) and AFM, ACM, AGS, GCO, FIN, GEI, HGI, NPA, GFN, DMP and AER (the blue-colored points in Figure 19p. Moreover, a smaller cluster contains DMG, GCO, GST, BAT, CCN, GBC, IBC, NTD, and TCL (the green-colored points in Figure 19). We can see that, except for a few points, the two main clusters that are visible in the heat kernel analysis are also well represented in our persistent components tree, although the bigger cluster is not seen in the tree as containing the two sub-clusters as separate subtrees, but rather mixing them.

To avoid problems due to the incompleteness of the SSWL database and the presence of poorly mapped languages, we filter the SSWL data by restricting the set of languages to only those that are at least $50 \%$ mapped. The persistent connected components analysis shows a very different clustering structure in this case, see Figure 20. It contains 3 main subtrees, corresponding to cluster N. 222, N. 238 and N. 203 (see Figure 20) but it also shows a lot more substructures, as opposed to only one main subtree and mostly singletons in the LanGeLin data set.

When comparing to the clustering of SSWL syntactic features obtained in [31] using the heat kernel method, we see that, except for two of the syntactic variables involved (which appear in the tree as clusters N. 206 and N. 203), all the features involved in the relations obtained in [31] are contained in the subtree corresponding to cluster N. 222 (colored points in Figure 21). Using the heat kernel method there are two main clusters that are formed; at $\epsilon=15$ with Neg06-Neg10, and at $\epsilon=20$ with Neg06-Neg10 and new points that are added, which are N209-N211, N203, N204, N206, Neg11-Neg14. In our persistent connected components tree the points from the $\epsilon=15$ case are clustered together (pink-colored points in Figure 21) and the points from the second cluster $(\epsilon=22)$ are also mostly grouped together (green-colored points in Figure 21). Thus, it seems that the relations obtained via 


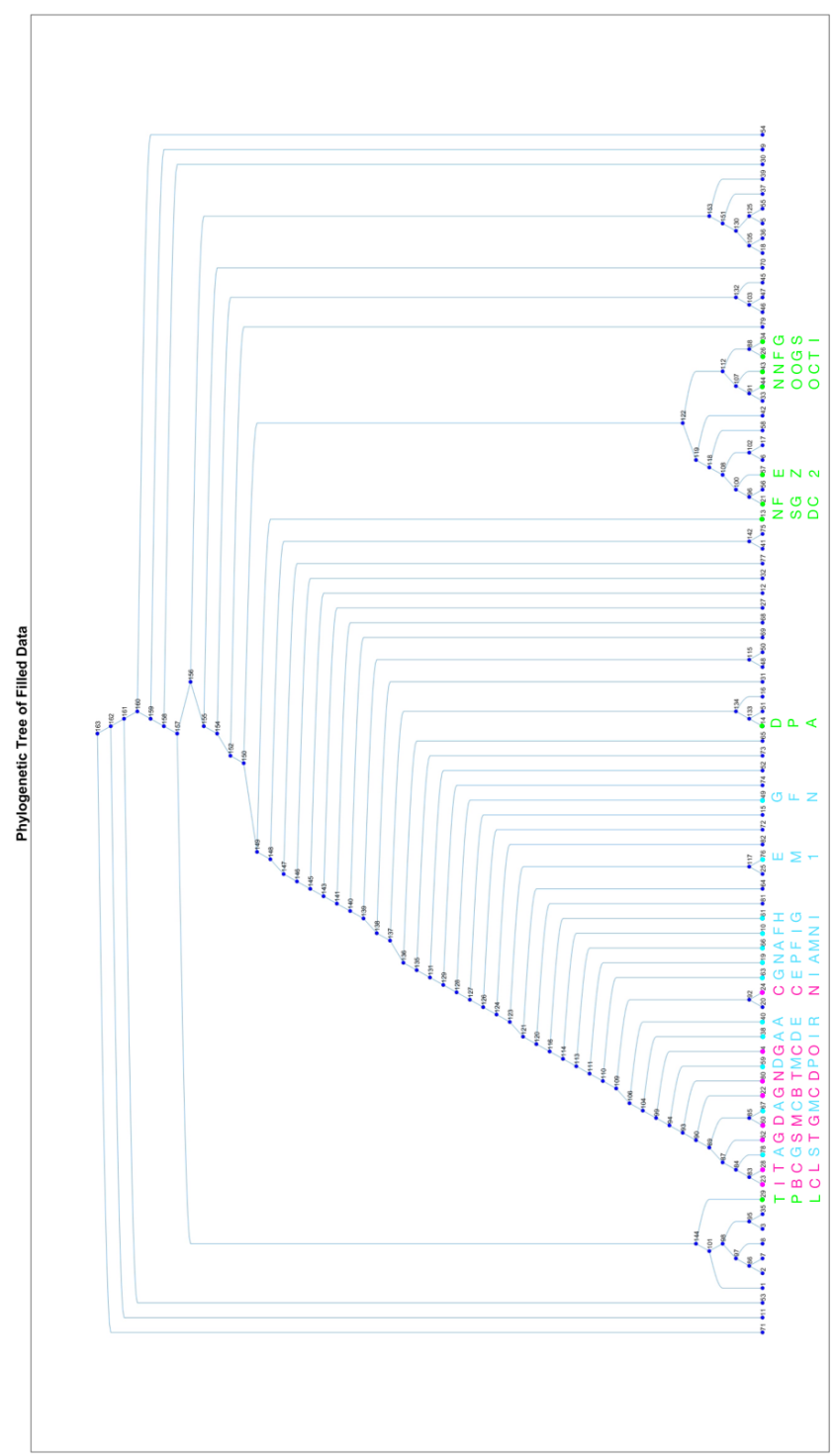

Figure 19. Persistent connected components tree for the syntactic parameters of the Longobardi data set and comparison with heat kernel clusters. 


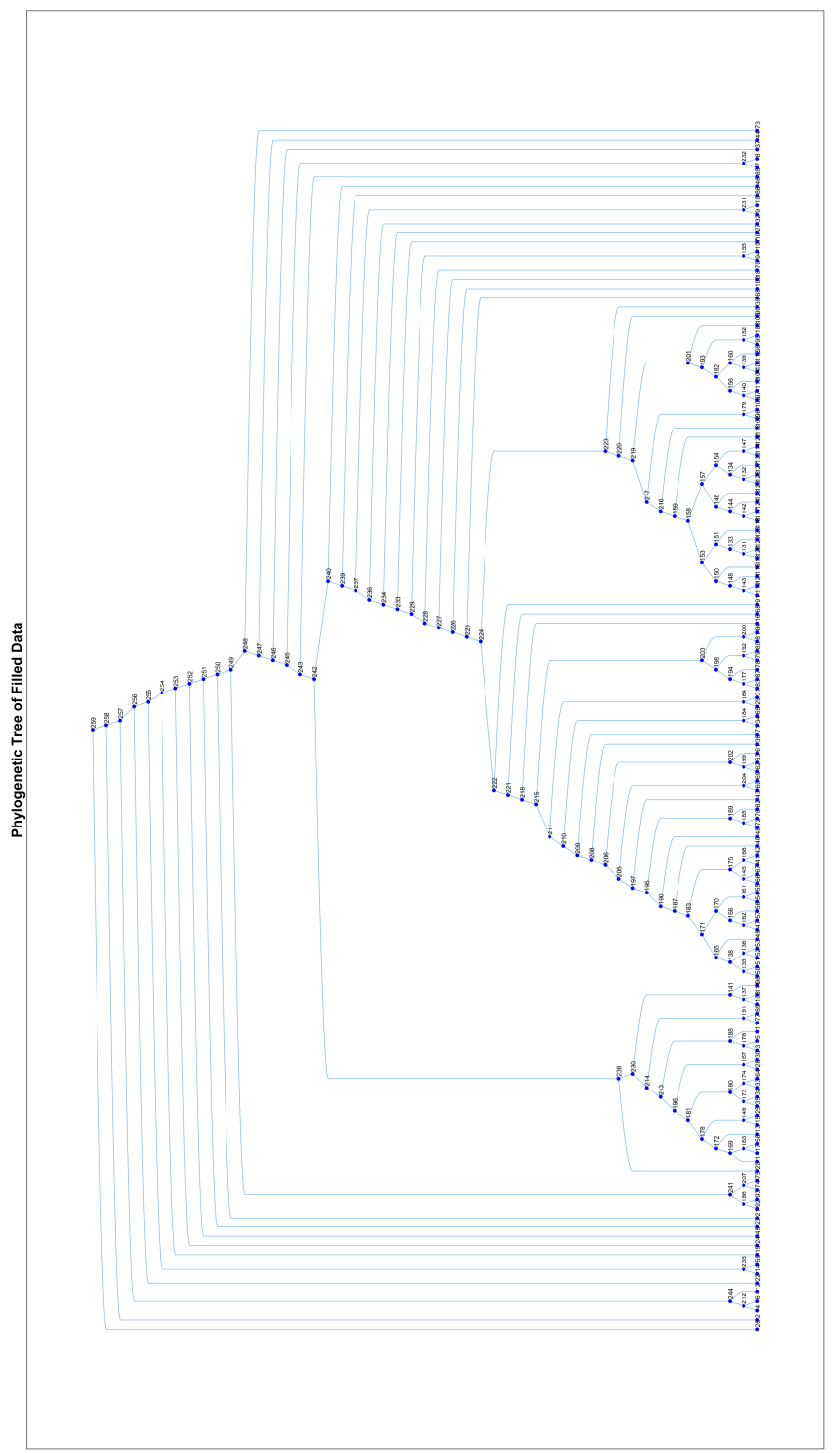

Figure 20. Persistent connected components tree for the syntactic variables of the SSWL data set. 


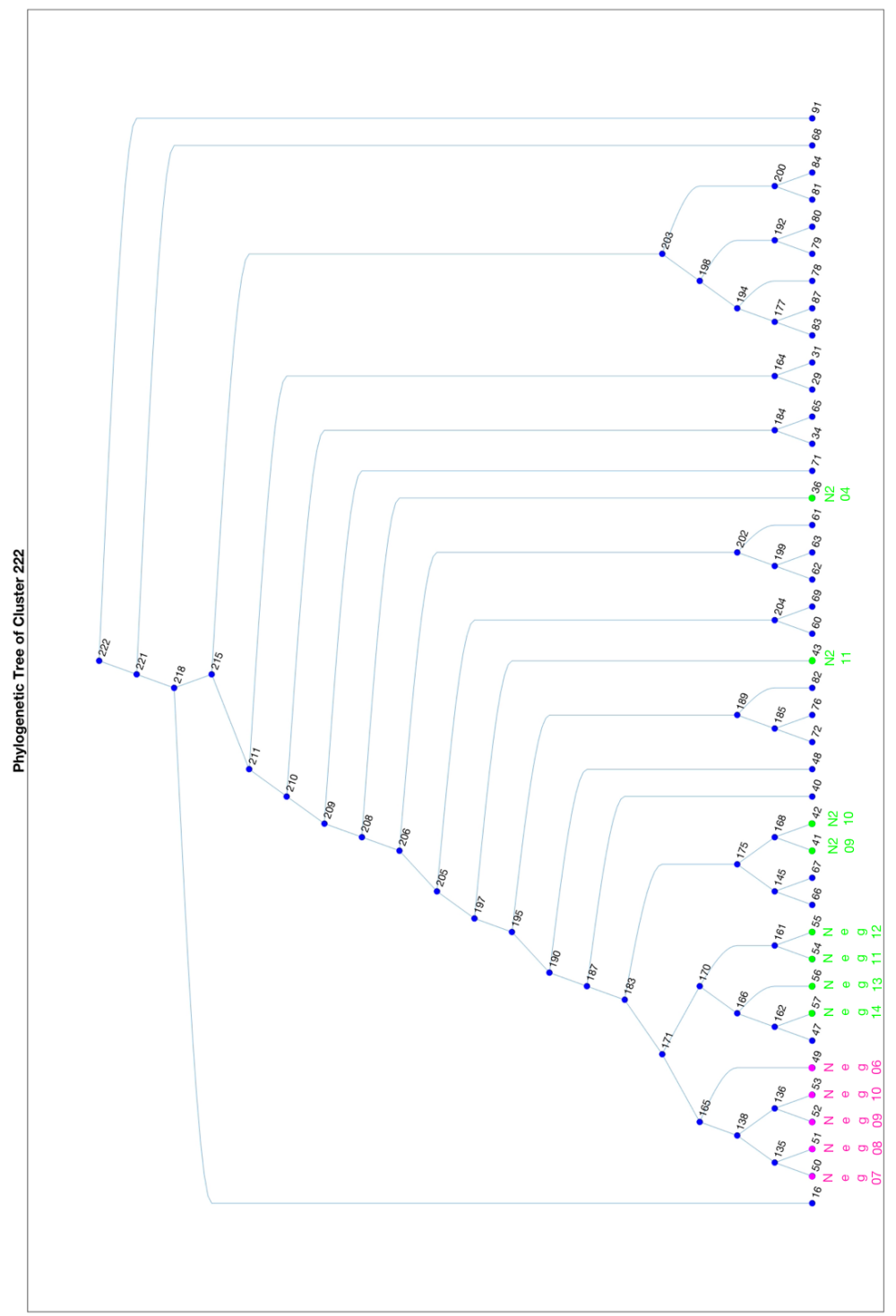

Figure 21. Persistent connected components tree for the syntactic variables of the SSWL data set, cluster N. 222, and heat kernel clusters. 
the tree of persistent connected components capture the clustering information obtained via the heat kernel method. The tree structure contains additional information that was not seen by the heat kernel analysis.

5.3. Comparison with machine learning methods. In [18] machine learning methods are used to construct a dependency graph for the syntactic parameters of the LanGeLin data, in which each parameter is represented by a vertex and relations between the parameters are represented by directed edges in the following way: if a parameter $x$ can be predicted by knowing all values of parameter $y$ then there is an edge from $y$ pointing to $x$.

The relation investigated in [18] can be of a different nature from the kind of alignment relations that the persistent $H_{0}$ (or to some extent the heat kernel method) detect. Indeed the graph of relations obtained in [18] also involves entailment relations where the value of one parameter may force another parameter to either anti-align or to become undefined. This type of relations would not be visible through the persistent connected components.

Moreover, another difference is the fact that the method of [18] identifies relations that hold "exactly" over the entire set of languages in the database, while the relations identified by the topological connected components are proximity relations that hold "approximately" over a sufficiently large subset of languages. In other words it is the difference between saying that the data points lie exactly on a submanifold defined by the relations or that the relations define a submanifold that interpolates the data with some precision (the data points are close to the submanifold but not necessarily on it).

Indeed we see for example that pairs of parameters in the graph of relations of [18] that have an arrow between them in both directions, such as $\{\mathrm{GCN}, \mathrm{GFN}\},\{\mathrm{FGN}, \mathrm{GCO}\}$ and $\{\mathrm{DMP}, \mathrm{DMG}\}$ correspond, respectively, to nodes number $\{48,49\},\{3,4\}$ and $\{59,60\}$ in our tree of persistent connected components, but these nodes are not placed in close proximity to each other in the tree.

5.4. First homology of the space of syntactic variables. The computation of the persistent topology barcode diagrams for the SSWL data is computed after filtering the data as indicated above. The barcode diagrams show the presence of non-trivial persistent generators of the $H_{1}$ homology group, though no higher dimensional persistent homology $H_{k}$ for $k \geq 2$ is detected.

Figure 22 shows the barcode diagrams (truncated at a certain scale) for the SSWL syntactic variables data (restricted to the Indo-European languages) and for the LanGeLin data (all languages). It appears that the space of the SSWL variables exhibits more persis-

tent $H_{1}$-generators than the space of the LanGeLin syntactic parameters. We will leave to future work to carry out a more in depth investigation of these $H_{1}$-generators and the linguistic significance of the relations between syntactic variables that they represent. We will also be discussing elsewhere the relations between syntactic parameters, detected through the persistent $H_{0}$ and $H_{1}$ for relations that are specific to certain language families. The presence of such family-specific syntactic relations is indicated by our dimensional analysis.

\section{LANGUAGE RELATEDNESS TREES FROM PERSistent COMPONENTS}

We now consider again our data sets so that data points are languages and their coordinates are given by the values of syntactic variables/parameters for a given language. 


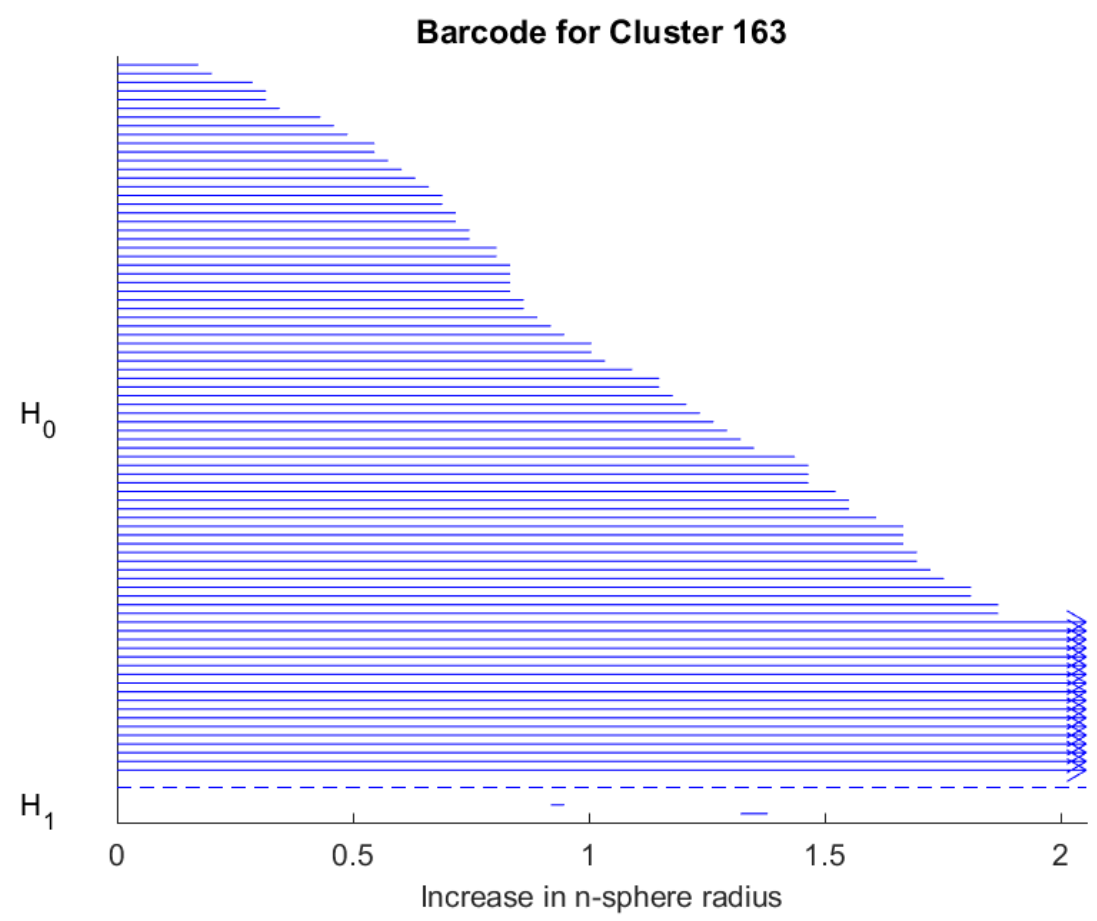

(A) SSWL data

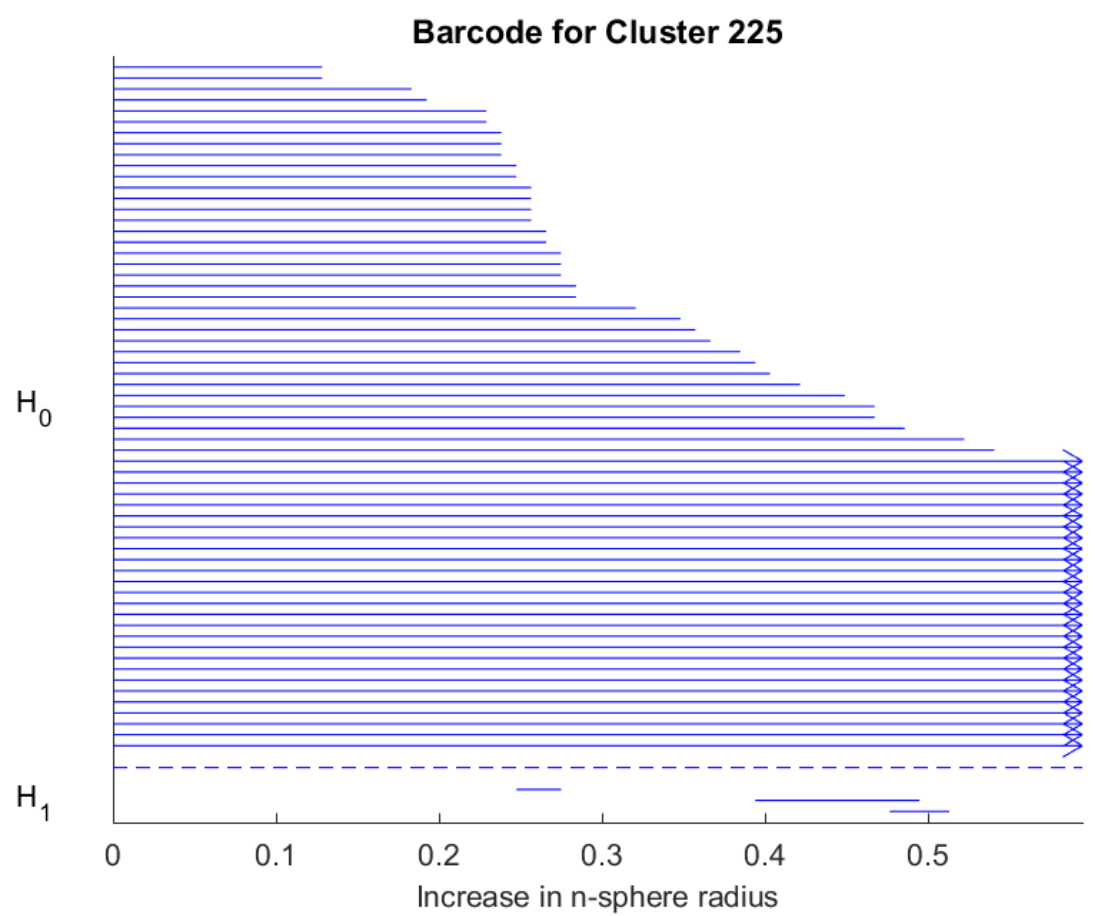

(B) LanGeLin data

Figure 22. Truncated barcode diagrams for the data of syntactic features (syntactic parameters) for the SSWL and the LanGeLin data showing persistent $H_{1}$. 
One of the main goals of the present investigation is to test whether the persistent components (persistent $H_{0}$ ) in the topological data analysis provide a valid independent method for the computational reconstruction of phylogenetic trees of language families. We will show here that the trees obtained from the persistent connected components are closely related to phylogenetic trees, in the sense that they largely provide an accurate splitting into subfamilies, but they also have significant differences. In particular, while in the usual phylogenetic trees of language families one should interpret the inner nodes of the trees as ancient languages that are ancestors to the modern languages, in the case of trees arising from the persistent connected components the inner nodes indicate the hierarchical structures of clusters, which is related to the subdivision into subfamilies, but does not represent an actual branching of an evolutionary process. In particular, the inner nodes cannot in general be identified with individual ancestors in the form of ancient languages or proto-languages, although we will see some specific examples where such an interpretation applies. In general, the inner vertices of our trees only represent a hierarchical structure of clustering of syntactic features.

Given the family of Vietoris-Rips complexes associated to the data at varying scales, one can compute the corresponding zeroth homology $H_{0}$. This counts the number of connected components of the Vietoris-Rips complex at that scale. Clearly, for sufficiently small scales, each data point will be a separate connected component, hence there is simply a different generator of the $H_{0}$ for each data point and no higher dimensional homology. At the other end of the interval of scales, for sufficiently large scales, all the points in the data set will be contained in a single ball of diameter the given scale around any of the points. In this large scale range the Vietoris-Rips complex consists of a single simplex of dimension equal to the total number of data points. This is a connected and contractible space hence its $H_{0}$ has a single generator and all the higher homology vanishes. In between these two extremes, the different singletons of the low scale picture begin to merge, in a certain order at certain values of the scale parameter, where the Vietoris-Rips complex correspondingly jumps by adding 1-simplices and possibly higher dimensional simplices as well. One can compute a tree that takes care of the order in which points are joined together by simplices in the Vietoris-Rips complex as the scale increases. The question is then whether the tree obtained in this way is closely related to the phylogenetic tree of historical language change within specific language families.

Since vertical relations between the languages are not reflected in the persistent components tree, which accounts for the hierarchical merging of clusters only, how to interpret the location of the old languages is a question by itself. Old languages appear as leaves of the tree rather than as root vertices of certain subtrees, so when comparing the persistent components trees with phylogenetic trees we should imagine them as lying above the other languages in their subtrees of the previous sub-clusters they merge with.

6.1. Various computational methods for phylogenetic trees. The reconstruction of phylogenetic trees of language families is an integral part of historical linguists research since its beginning. In recent years, due to a vast research in the field of computational biology, more and more methods for phylogenetic tree reconstruction have been introduced. It is a point of interest for linguists, and other scientists alike, whether these methods can yield good results when applied to linguistic data, such as linguistic characters (lexical, morphological and phonological) and syntactic parameters. In this paper we are interested only in the latter type of data. The three main methods for tree reconstruction that 
have been used in this setting are distance based methods (such as neighborhood joining and UPGMA) and character based methods (such as maximum parsimony and maximum likelihood), as well as phylogenetic algebraic geometry.

- Distance based methods: a phylogenetic tree is reconstructed based on the distances between the data points. Two very well known distance based methods are the neighbor joining method and UPGMA (Unweighted Pair Group Method with Arithmetic Mean).

- Character based methods: a phylogenetic tree is reconstructed using a character table which describes the data. The goal is to reconstruct a tree where similar character values occur near each other. The most used methods here are Maximum Parsimony and Maximum Likelihood.

- Phylogenetic Algebraic Geometry: a phylogenetic algebraic variety is associated to a hidden Markov model on a tree, with the data providing the probability distribution at the leaves (the visible nodes). The phylogenetic invariants (generators of the ideal defining the phylogenetic variety) are used to evaluate how well the data fit the model.

Phylogenetic algebraic geometry is the study of algebraic varieties derived from phylogenetic trees (which are usually associated with an evolution of biological sequences), see [32]. In algebraic phylogenetics, a tree $T$ is associated with a geometric object $\left(V_{T}, x_{T, \mathcal{P}}\right)$ where $V_{T}$ is an algebraic variety determined by the topology of the tree $T$ and $\mathcal{P}$ is a boundary distribution on the leaves of $T$, which is a polynomial function of the model parameters. The point $x_{T, \mathcal{P}} \in V_{T}$ lies on the sublocus $V_{T}\left(\mathbb{R}_{+}\right) \subset V_{T}(\mathbb{R})$. In some cases, when $V_{T}$ is a classical well studied algebraic variety, it is possible to get an interesting information about underlying structures of $T$ from the sub-variety $x_{T, \mathcal{P}}$ lies on. For example, it can provide a connection between the distribution $\mathcal{P}$ and the splittings of the tree into sub trees. In the process, trees are generated using some algorithms (such as DNA parsimony algorithms), and using algebraic phylogenetic methods, phylogenetic invariants are calculated. Using these invariants and estimates of the Euclidean distance in the ambient affine space between the point $\mathcal{P}$ and the phylogenetic variety $V_{T}$, a best candidate tree is chosen. As shown in [37, trees that were reconstructed via this method from SSWL and LanGeLin data were correctly corresponding to the actual phylogenetic trees known to historical linguists. The authors also argued that this method is better at generating phylogenetic trees from syntactic parameters than other distance based methods.

There is some controversy over the use of computational methods for the reconstruction of language phylogenetic trees, especially for the Indo-European family, see [34]. In [39] the authors discuss the advantages of the algebro-geometric approach used in [37] over other distance based methods. It is shown that the Algebro-Geometric approach yields better results, especially when additional information, such is the subdivision into subfamilies and the position of the old languages is taken into account. We discuss here our construction of persistent connected components trees and their comparison with known phylogenetic trees.

It is important to stress here the fact observed in [37] that historical phylogenetic trees can be correctly reconstructed from the (filtered) SSWL data and the LanGeLin data, since this implies that the discrepancies we will outline below between the trees we construct using persistent connected components and the phylogenetic trees are not due to some inadequacy of the data, but show a genuine intrinsic difference between the information encoded in the 
hierarchical structure of the clustering of the connected components in the persistent $H_{0}$ and the trees of historical language development.

6.2. Persistent components trees and language relatedness. In this section we present a detailed analysis of persistent components trees for both the SSWL and the LanGeLin data sets, where data points are languages, with their coordinates given by the values of their binary syntactic variables (SSWL) or syntactic parameters (LanGeLin). This procedure produces trees that reflect the merging of the persistent components as the scale parameter of the Vietoris-Rips complex increases, and provide a measure of language relatedness seen at the syntactic level. We discuss to what extent the trees obtained in this way reflect historical phylogenetic trees of language families. We apply our same tree construction algorithm described in Section 5.1.

Other methods of computational phylogenetics were used to produce phylogenetic trees of language families based on syntactic data [19], 21], [22], [23], and also [37], as well as on a combination of syntax and other linguistic data, [24], [36]. What is new in the analysis we present here is not the use of syntactic data but the method for tree reconstruction, which is based on persistent connected components rather than on other computational methods of tree reconstruction. As mentioned above the resulting trees should not be read exactly as phylogenetic trees, although we will see that they do contain phylogenetic information.

In Section 6.1 we analyze in more detail the different methods for phylogenetic tree reconstruction in comparison to ours, whereas in the rest of this section we focus on the results obtained with our method.

6.3. Persistent components trees of Indo-European languages. We use separately the two different data sets, the SSWL and the LanGeLin syntactic data, to build our persistent components trees. Although the SSWL contains more languages, the languages are not uniformly mapped in the present version of the database. The LanGeLin data set on the other hand contains fewer languages but nearly all of the languages are fully mapped (in ternary rather than binary variables), and with variables that more accurately correspond to the linguistic notion of "syntactic parameter". Indeed, as was also observed in [37] in tree reconstructions obtained via the phylogenetic algebraic geometry method, the trees generated using the LanGeLin data appear to reflect more accurately in their topology and clustering what is known from historical linguistics about phylogenetic trees of subfamilies of the Indo-European families.

It was shown in [39] that if one attempts a phylogenetic tree reconstruction using the entire SSWL database simultaneously with the lacunae in the data simply filled by a 0.5 value one obtains nonsensical answers regarding language proximity in the tree, with languages misplaced within their family, or placed in a completely different family altogether, and with ancient languages especially likely to be misplaced. Although the computational method for phylogenetic reconstruction used in [39] to illustrate this point was simply the standard parsimony method built into the PHYLIP phylogenetic software, the same problem is likely to affect the trees obtained via our topological persistent components as well.

Indeed, we will first compute the outcome of applying our method to the entire IndoEuropean family of SSWL data, retaining all languages, without filtering them by completeness. We will then analyze where the tree constructed in this way fails to represent faithfully the historical linguistic information. We will then proceed to analyze smaller subfamilies by filtering the languages by completeness and proceed to a more accurate tree reconstruction 
which we will again compare to known historical linguistic information. We will show that, although this filtering of the SSWL data improves the correlation between the persistent components trees and the phylogenetic trees, they still show significant differences.

6.3.1. Indo-European tree from the full unfiltered set of SSWL data. First let us view the tree generated from the SSWL data. When we use the full data, including the poorly mapped languages, we obtain a tree that has one main big subtree (cluster N. 133) and the other subtrees represent either small clusters (with maximum of 4 points) or singletons that are joined later on. This tree splits into two main subtrees. One is cluster N. 129 which in turn splits into cluster N. 125 that contains most of the Romance languages and cluster N. 124 which contains most of the Germanic languages. The other is cluster N. 132 which contains a mix of many different language subfamilies such as some of the Greek languages, Altaic languages, Balto-Slavic languages, some Indo-Iranian languages, and more. Other than that, in a close proximity to this subtree (N. 130) we have the Armenian languages and Ossetic languages (Indo-Iranian) grouped together in subtree 134. This may support the hypothesis of Proto-Armenian situated between Proto-Greek and Proto-Indo-Iranian. As we know that although Armenian languages form a branch by themselves they share common features with Indo-Iranian and Greek languages. Most of the other languages are added as singletons (this was discussed in Section 2).

However, as we discuss below in the examples of specific language subfamilies of the IndoEuropean languages, the use of the full unfiltered data including poorly mapped languages and incomplete variables causes a lot of incorrect placements of languages both within and across subfamilies, as already observed with other tree construction methods in [39]. Thus, we restrict the SSWL data by first keeping only languages that are at least a certain percentage complete and then for those languages we keep only the syntactic variables that are fully mapped, as done in [37].

6.3.2. Persistent components tree from the filtered $S S W L$ data. We consider the subset of languages of the SSWL database that are at least 50\% complete and we restrict their coordinates to the subset of syntactic variables that are completely mapped for all the languages in this set. We then compute the persistent components tree with the method discussed above. The resulting tree is illustrated in Figure 23. We focus on the IndoEuropean languages together with the Uralic and Altaic languages (we include also Korean and Japanese although no longer regarded as plausibly Altaic).

In the cluster structure of the tree of Figure 23 we see (from left to right) a cluster N. 63 with Korean and Japanese which is a reasonable clustering. Cluster N. 92 contains the Armenian languages (Eastern Armenian and Western Armenian joined together as subcluster N. 62), successively joined by Turkish, Hungarian, and Cappadocian Greek. This clearly does not correspond to a historical phylogenetic clustering since it mixes some IndoEuropean and some Uralic and Altaic languages. Cluster N.45 is a singleton with Sorani Kurdish, which only joins other components very high up in the tree (cluster N. 93). Cluster N. 68 is again a cluster that has good correlation with historical phylogenetic trees: it contains the Indo-Iranic Indo-European languages Hindi, Panjabi, Pashto, and Nepali. Cluster N. 60 only contains English and Singaporean English and is also added very late to the other components (cluster N. 89): in particular, even after filtering in order to correct for the incompleteness of the data, when all language families are considered together, English is not correctly grouped near the other Germanic languages. Clusters N. 48 and N. 34, two 


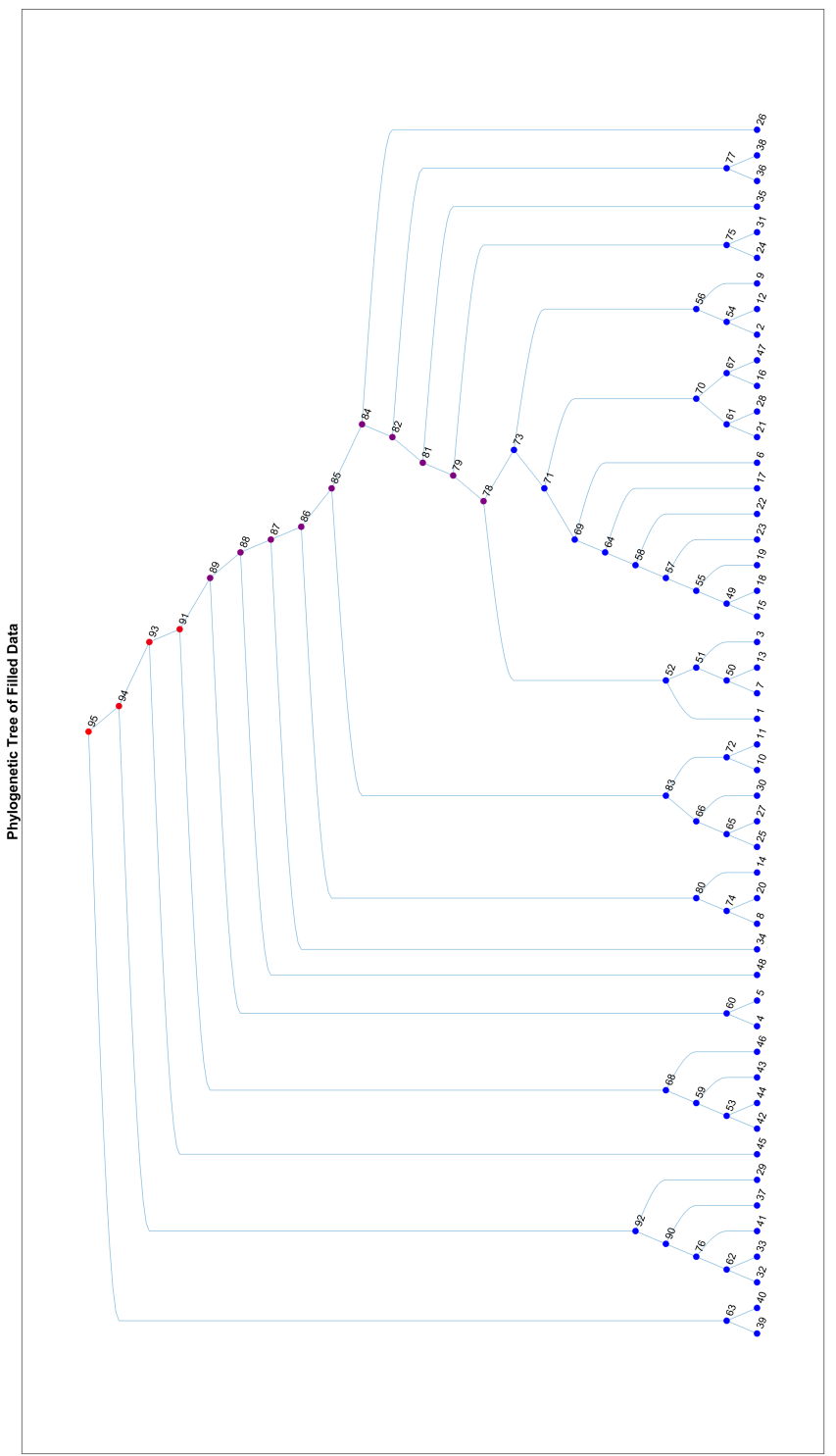

FIgURE 23. Persistent components tree for the filtered SSWL data. 

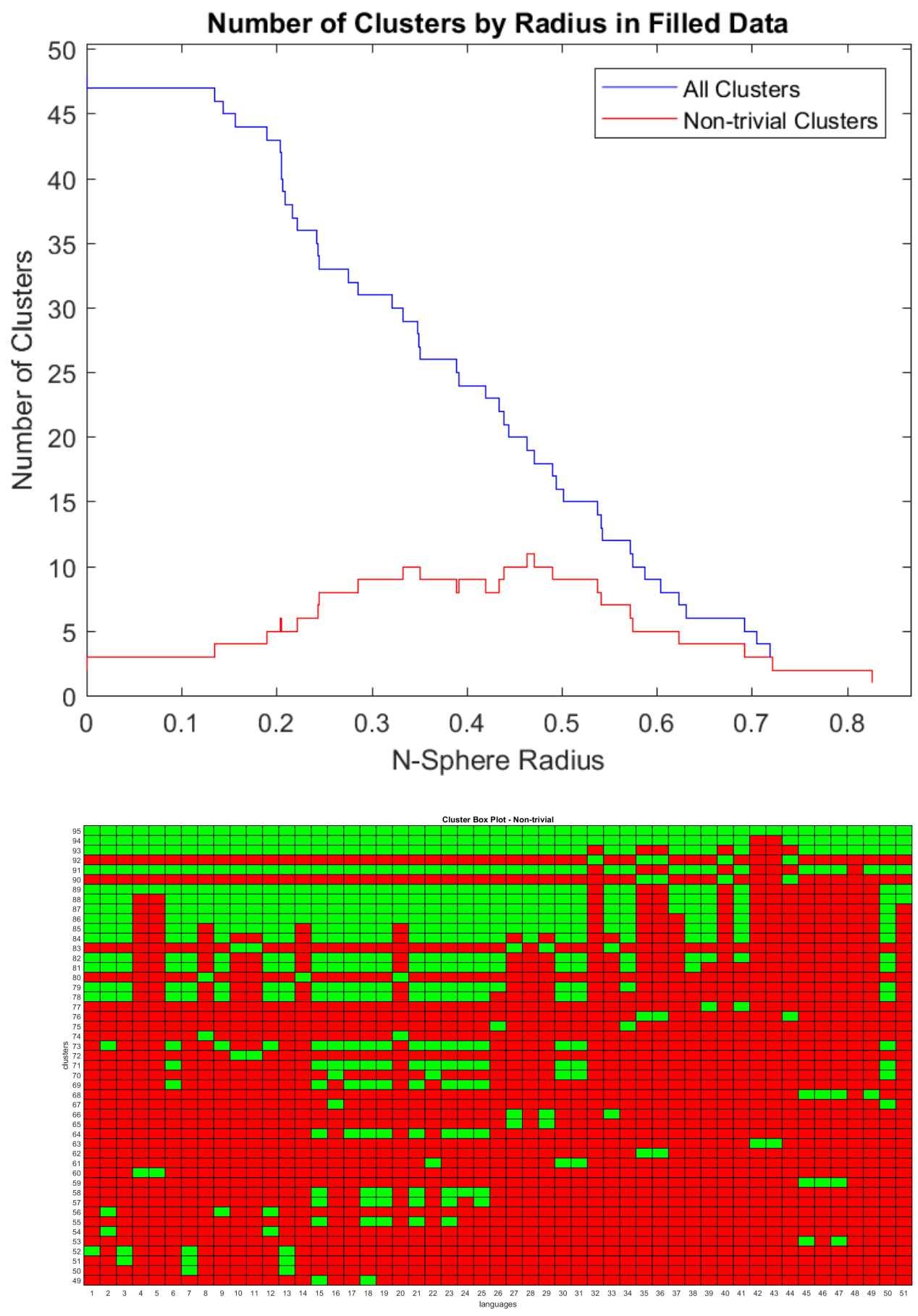

Figure 24. Clusters and clustering structure in the tree of the filtered SSWL data. 
singletons, respectively consisting of Haitian and Czech, which also join the tree at higher levels: again these languages are misplaced out of grouping within the appropriate subfamily. Cluster N. 80 contains Gothic, Icelandic, and Late Latin. This is an indication of the tendency of the SSWL data, when analyzed without prior subdivision into subfamilies, to misplace the location of the ancient languages. This fact was also already observed by different methods in [39]. Cluster n. 83 again groups together mostly ancient languages: Latin, Ancient Greek, and Homeric Greek (sub-cluster N. 66) and Old English and Old Saxon (sub-cluster N. 72). It is not surprising that, without additional indication about subfamilies, the syntactic similarities between the ancient Indo-European languages is detected as a closer clustering than the respective similarities with their modern descendants. Cluster N. 52 contains some Germanic languages: Afrikaans, Dutch, German, and West Flemish, all of which belong to the West-Germanic split of the Germanic languages. So again we see here a subtree that correlates well with historical phylogenetic trees (except for the misplacement of English outside of this tree as we mentioned above). This cluster N. 52 is part of a larger cluster (N. 78) which contains another main subtree (cluster N. 73) which in turn splits into three main sub-clusters, N. 69, N. 70, N. 56. Of these, cluster N. 56 contains other Germanic languages all belonging to the North-Germanic split: Danish, Norwegian, and Swedish. Two other North-Germanic languages in the database, Icelandic and Faroese are misplaced: Icelandic, as we have seen, is grouped with Gothic and Late Latin, and Faroese is in the same larger cluster N. 78 that contains the Germanic languages, but placed in closer proximity to the sub-cluster (N. 69) that contains the Romance languages Portuguese, Brazilian Portuguese, Catalan, Italian, Napoletano Antico, Sicilian, Spanish, and French. The remaining sub-cluster in the larger cluster N. 78 is cluster N. 70 that contains Northern Calabrian, Romanian, Cypriot Greek, Greek, and Albanian. This sub-cluster again does not correspond to a historical phylogenetic tree since it contains a mixture of Hellenic and Romance languages. Cluster N. 75 has Galician and Medieval Greek, which again do not have close historical relatedness. Cluster N. 35 has Polish that appears as a singleton, while Russian appears in the nearby two-point cluster N. 77 joined with the Uralic language Finnish. After filtering the data for completeness no other Slavic language is left. The last singleton cluster, N. 26 contains Old French.

The clustering structure of the tree of the persistent connected components clearly does not reflect the branching into main families (there is a mixing of Indo-European and Uralic and Altaic languages for instance) nor the splitting into subfamilies of the Indo-European language family, even though it retains some information about the subdivision into subfamilies. As we discuss more in detail below when we focus on smaller subfamilies of the Indo-European family, this discrepancy between persistent components trees and historical phylogenetic trees is not caused by a problem with the SSWL data. Indeed we will see that the discrepancy persists when we focus on smaller groups of languages for which it was already established in [37] that the SSWL data do reconstruct the correct historical phylogenetic tree, when the phylogenetic algebraic geometry method is used for the inference of the tree structure. Thus, the discrepancy with the persistent components tree should be ascribed more intrinsically to a different type of information about syntactic proximity carried by the clustering of the persistent $H_{0}$. We will show later that some amount of misplacement at smaller scales (within subfamilies but nor across larger family subdivisions) can also be caused by the variance level in the PCA analysis: this may also account for some of the misplacements within the smaller subfamilies. 


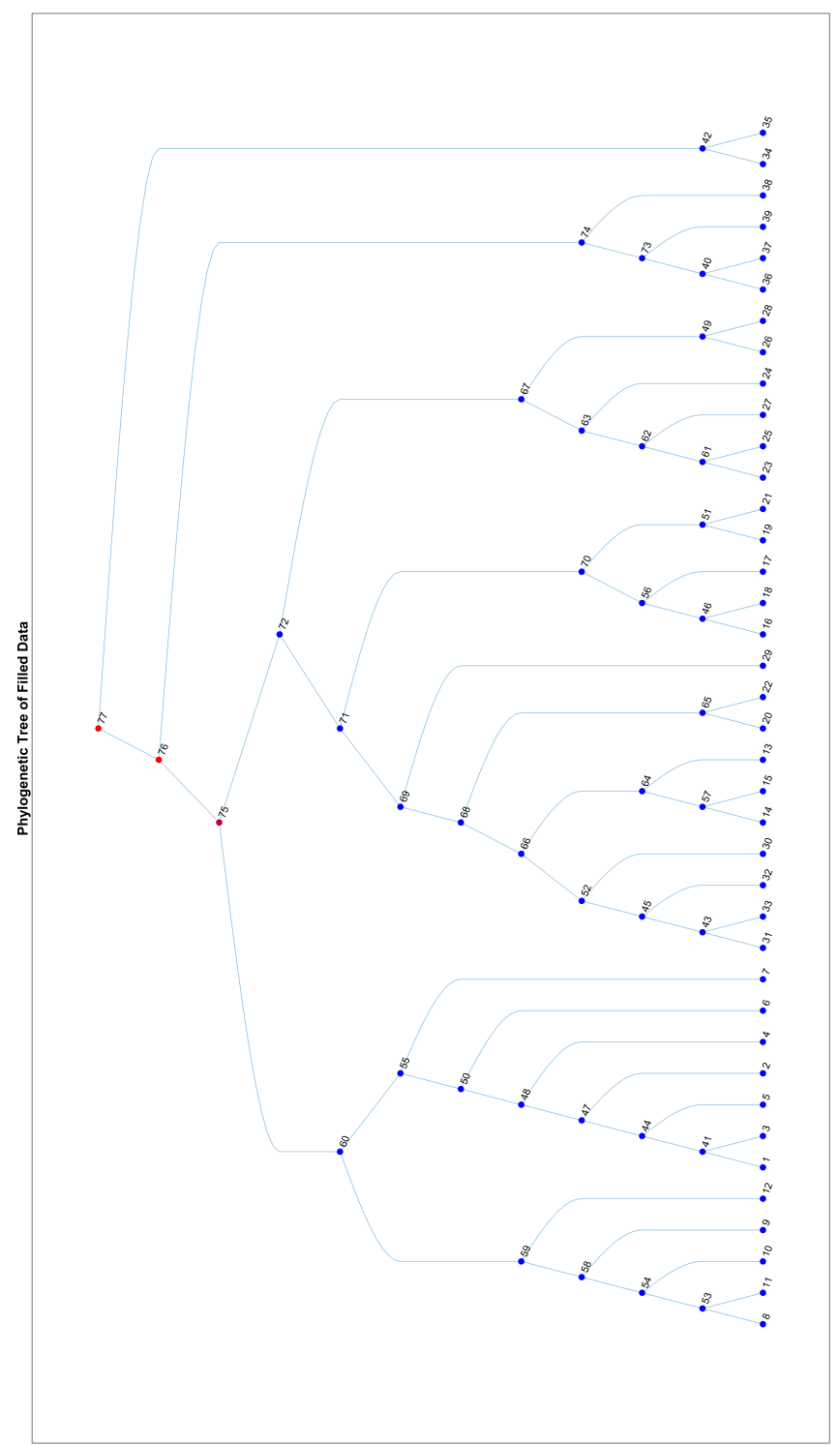

Figure 25. Persistent components tree from LanGeLin data, PCA $60 \%$. 

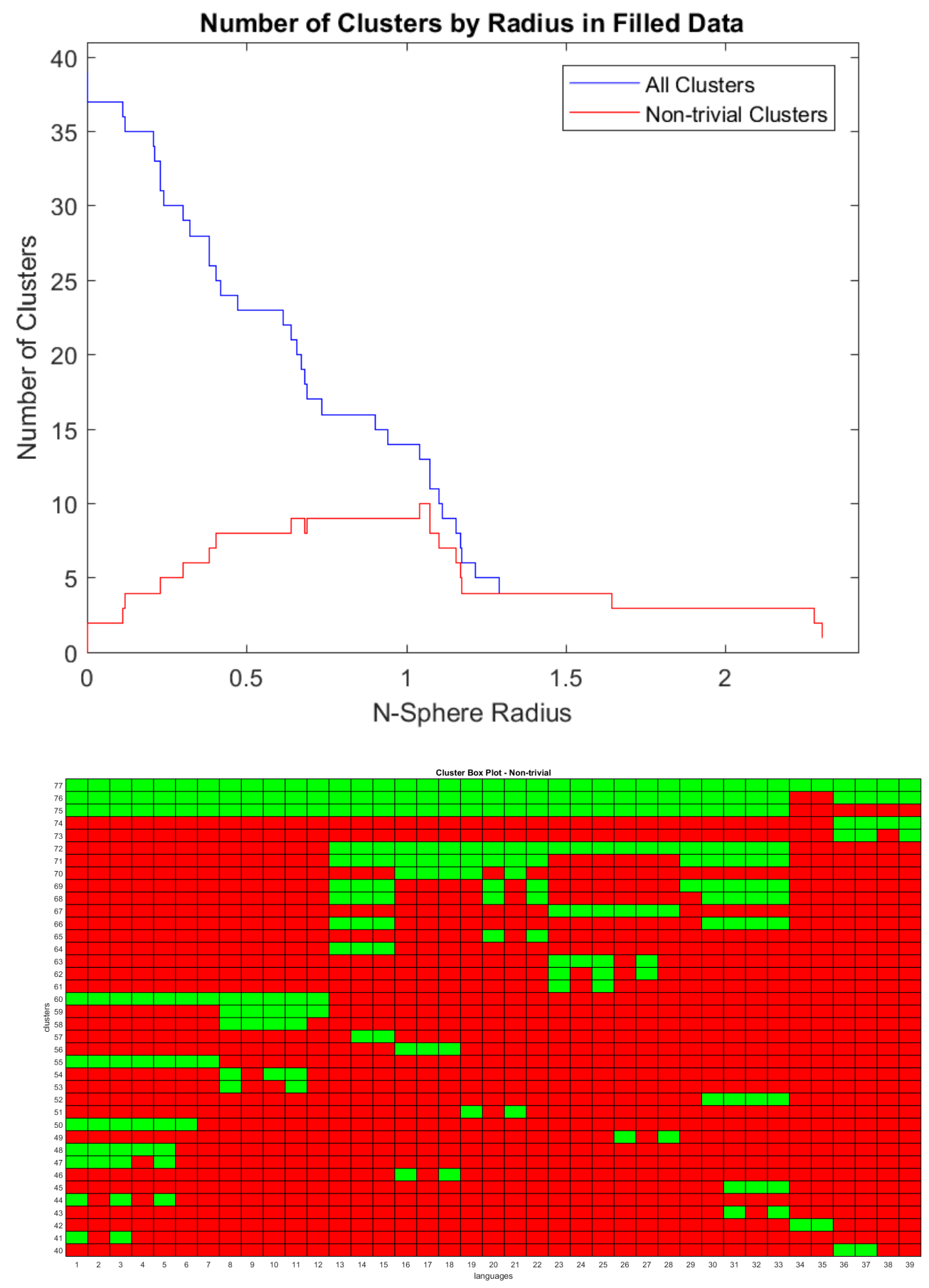

Figure 26. Clusters and clustering structure in the persistent components tree for the LanGeLin data, PCA $60 \%$. 
6.3.3. The Indo-European persistent components tree of the LanGeLin data. Our topological reconstruction of a persistent components tree for the LanGeLin data (see Figure 25) exhibits a stronger correlation between the clustering of the persistent connected components and the tree topology of the historical phylogenetic trees than the case of the tree of the SSWL data analyzed above. As discussed before (Section 2) the LanGeLin persistent components tree almost does not have any singletons (especially at PCA level of 60\%).

The structure of the subtrees (from left to right) gives the following clustering of languages. We have a large cluster (N. 60) that contains two main subclusters, N. 59 and N. 55. Cluster N. 59 contains the main modern Romance languages: Italian, Spanish, French, Portuguese, and Romanian, while the nearby cluster N. 55 contains the Romance Southern Italian dialects: Ragusa, Mussomeli, Aidone, Southern Calabrese, Salentino, Northern Calabrese, and Campano. There is then another very large cluster, N. 72, which contains several sub-cluster structures which include Hellenic, Germanic, and Slavic languages. In particular, cluster N. 52 contains all the Slavic languages except Bulgarian: Serb-Croatian, Slovenian, Polish, and Russian. Cluster N. 64 groups together the ancient languages Latin, Classical Greek, and New Testament Greek. Cluster N. 65 has Romeyka Pontic Greek grouped together with Gothic and adjacent to the previous cluster with ancient Greek. We will return to discuss the association of Gothic and the Hellenic languages in relation to $H_{1}$-structures later. Cluster N. 29 contains Bulgarian as a singleton that is added to the previous clusters (N. 52 Slavic languages, N. 64 and N. 65 ancient languages). As we discuss more in detail below, this occurrence of a mixture of Hellenic and Slavic languages together with Gothic seems to be related both to the $H_{1}$-structure that we observed in 35 in the SSWL data and to an $H_{1}$-structure in the LanGeLin data that we will discuss more in detail in Section 7. This large cluster N. 69 is joined with cluster N. 70 to form the larger cluster N. 71. Cluster N. 70 contains the Hellenic languages including the Greek Southern Italian dialects of the Greek-Italian microvariations of 13: Salento Greek, Calabrian Greek A, Calabrian Greek B, grouped together with Modern Greek and Cypriot Greek. The large cluster N. 71 is then joined with cluster N. 67 that contains the Germanic languages Old English, English, Dutch, Danish, Icelandic, and Norwegian. As in the SSWL case, Icelandic is incorrectly placed: here it occurs together with the West-Germanic languages instead of the North-Germanic. The whole cluster structure N. 72 (Hellenic, Germanic, and Slavic languages) is joined to the previous cluster N. 60 of the Romance languages into cluster N. 75. This is then joined by the smaller cluster N. 74, which contains the Indo-Iranian languages Marathi, Hindi, Farsi, and Pashto, followed by a joining with the small cluster N. 42 with Irish and Welsh.

As opposed to the SSWL case, with the LanGeLin data all of the Germanic languages are grouped together within the same subtree, with Norwegian and Danish as a subtree (these correctly belong to the North-Germanic branch) and the rest (Old English, English, German, Icelandic and Goth) in a subtree together with the Balto-Slavic languages. Regarding the position of the old languages within the tree, we find Gothic located closer to the Balto-Slavic subtree than to the West-Germanic subtree and Icelandic erroneously located together with the West-Germanic languages. Despite the structure involving Gothic that we will discuss later, the old languages Gothic and Old English are correctly located in the same clustering structure involving the sub-cluster of the West Germanic languages.

However, the nearby grouping of the Germanic and the Balto-Slavic languages in the persistent component tree of the LanGeLin data differs from the usual phylogenetic tree 
of the Indo-European languages (including the one constructed from the LanGeLin data in [22]) which have an Indo-Iranian and Balto-Slavic split followed at a lower level in the tree by a Germanic split. The main branches in the tree of [22] derived from an earlier version of the LanGeLin data take the form

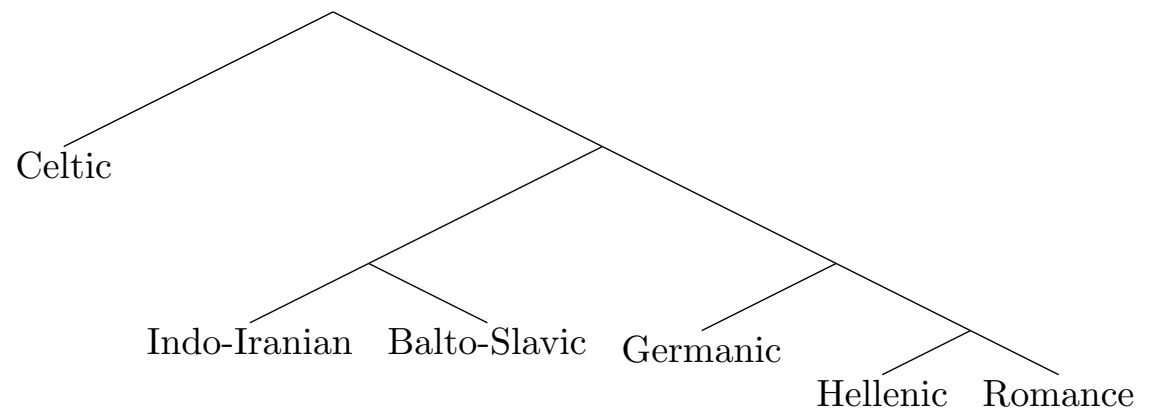

The persistent components tree for the full LanGeLin data presents a different branching structure between the Indo-European subfamilies, of the form

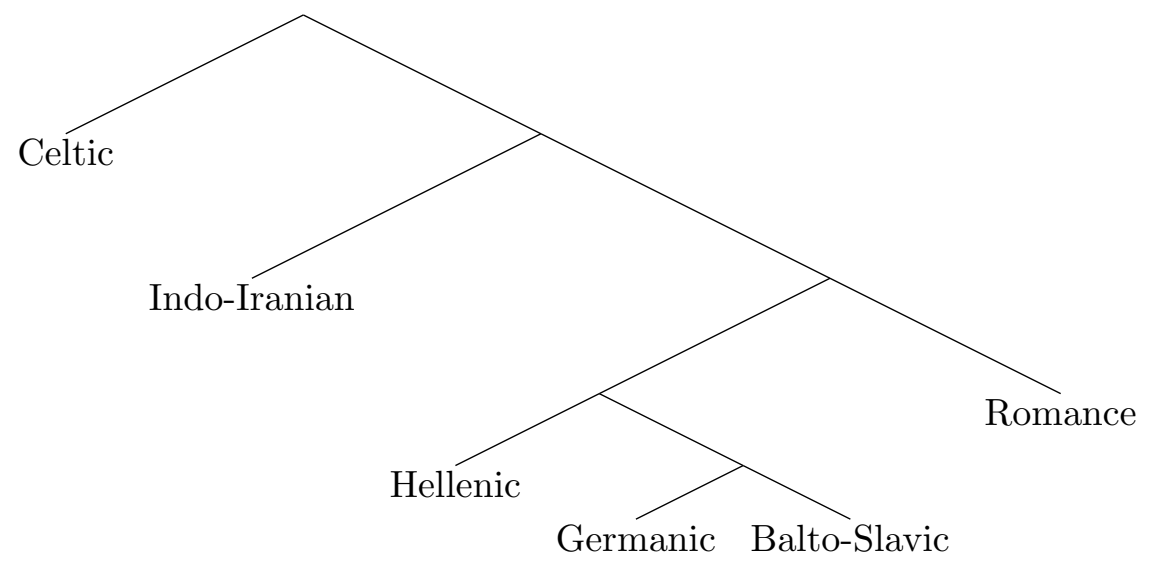

This subdivision differs also from other proposed phylogenetic trees of the main IndoEuropean language families. It is more likely that this signals the different meaning between the clustering information contained in the persistent components and the historical relatedness through phylogenetic trees rather than indicating a different possible phylogenetic proposal for the Indo-European family tree.

When computing the larger tree (also at PCA 60\%) for all the languages in the LanGeLin data, we also find a larger cluster that contains the Indo-Iranian languages as a sub-cluster, but now we find the non-Indo-European Uralic language Yukaghir grouped within the same sub-cluster with the Indo-Iranian languages. In proximity of this sub-cluster we find another sub-cluster with other Uralic languages, Hungarian, Finnish, Estonian, and Khanty. These Uralic languages are in turn placed next to the putative Altaic languages Yakut, Turkish, Buryat, Even, and Evenki. The remaining Uralic language Udmurt (Ud) appear together with the Altaic languages, see the more detailed discussion in Section 6.8. These sub-clusters are joined into a large cluster that also contains a singleton given by the Inuit language Inuktitut. Japanese (Jap) and Korean (Kor) are placed in proximity of each other but not in proximity of the Altaic languages, in agreement with the current understanding that they should not be considered part of a hypothetical Altaic or Ural-Altaic family. 
6.4. Germanic language family. First let us consider the Germanic family persistent components tree. It is known from historical linguistics that the Germanic language family consists of two main branches: the North Germanic branch and the west Germanic branch. We discuss the trees obtained by computation of the persistent connected components for the SSWL data and for the LanGeLin data and we compare them with the available information from historical linguistics. This is a good example where we see that the persistent components tree can differ from the phylogenetic tree in significant ways. It is also an example that illustrates that there can be significant effects caused by changing the level of variance of the PCA as we discuss below.

6.4.1. Germanic language family in the SSWL data. The Germanic languages recorded in the SSWL database include: Afrikaans, Cimbrian, Danish, Dutch, English, Middle English, Old English, Faroese, Frisian, German, Gothic, Icelandic, Middle Dutch, Mòcheno, Norwegian, Oevdalian, Old Norse, Old Saxon, Swedish, Swiss German, West Flemish, and Yiddish.

Looking at the full tree for the unfiltered SSWL data we see that that most of the Germanic language are grouped together in tree N. 124, but there are notable misplacements to other family trees, for example the case of English which is incorrectly located within the Romance languages subtree (tree N. 125). The tree N. 124 splits into two subtrees; The first is tree N. 119 containing Afrikaans, Faroese, Frisian, Icelandic, Norwegian, Swiss German and West Flemish and the second one, tree N. 114, containing Dutch, German, and Swedish. Swedish is misplaced in the same subtree with Dutch and German. While the latter two belong to the same West Germanic branch, Swedish is a North Germanic language. A further look into the second subtree containing the rest of the Germanic languages reveals that it splits into two branches, one consisting of West Frisian and Swiss German and the other containing a sub-branch of Faroese, Icelandic, Norwegian, and another sub-branch of Afrikaans and West Flemish. The positions of some of these languages do indeed correspond to the known phylogenetic tree, while other positions such as that of Swedish are clearly wrong, and some languages are entirely missing from the subtree, like English which is misplaced in a completely different family tree.

This further confirms that, although most of the German subfamily is grouped together, the position of some languages within this Germanic tree is incorrect with respect to its known position. This reflects indeed the same type of problems discussed in [39] regarding the reconstruction of phylogenetic trees using the entire unfiltered SSWL data.

To circumvent the problem of the incompleteness of the SSWL data, we proceed as before and we filter the set of Germanic languages by retaining only those that are at least $50 \%$ complete and then for this set we retain only those parameters that are completely mapped for all the languages in the set. This reduces the dimensionality of the ambient space. We then further select smaller sets of Germanic languages and use only syntactic variables that are fully mapped for all of these languages. The remaining languages in the Germanic family after this filtering are Afrikaans, Danish, Dutch, English and Singaporean English, Faroese, German, Gothic, Norwegian Old English, Old Saxon, Swedish, West Flemish, and Icelandic.

After retaining only the languages and fully mapped syntactic variables selected in this way, the persistent components tree that we obtain from the SSWL data for the Germanic languages (at PCA 60\%) is of the form illustrated in Figure 27. 


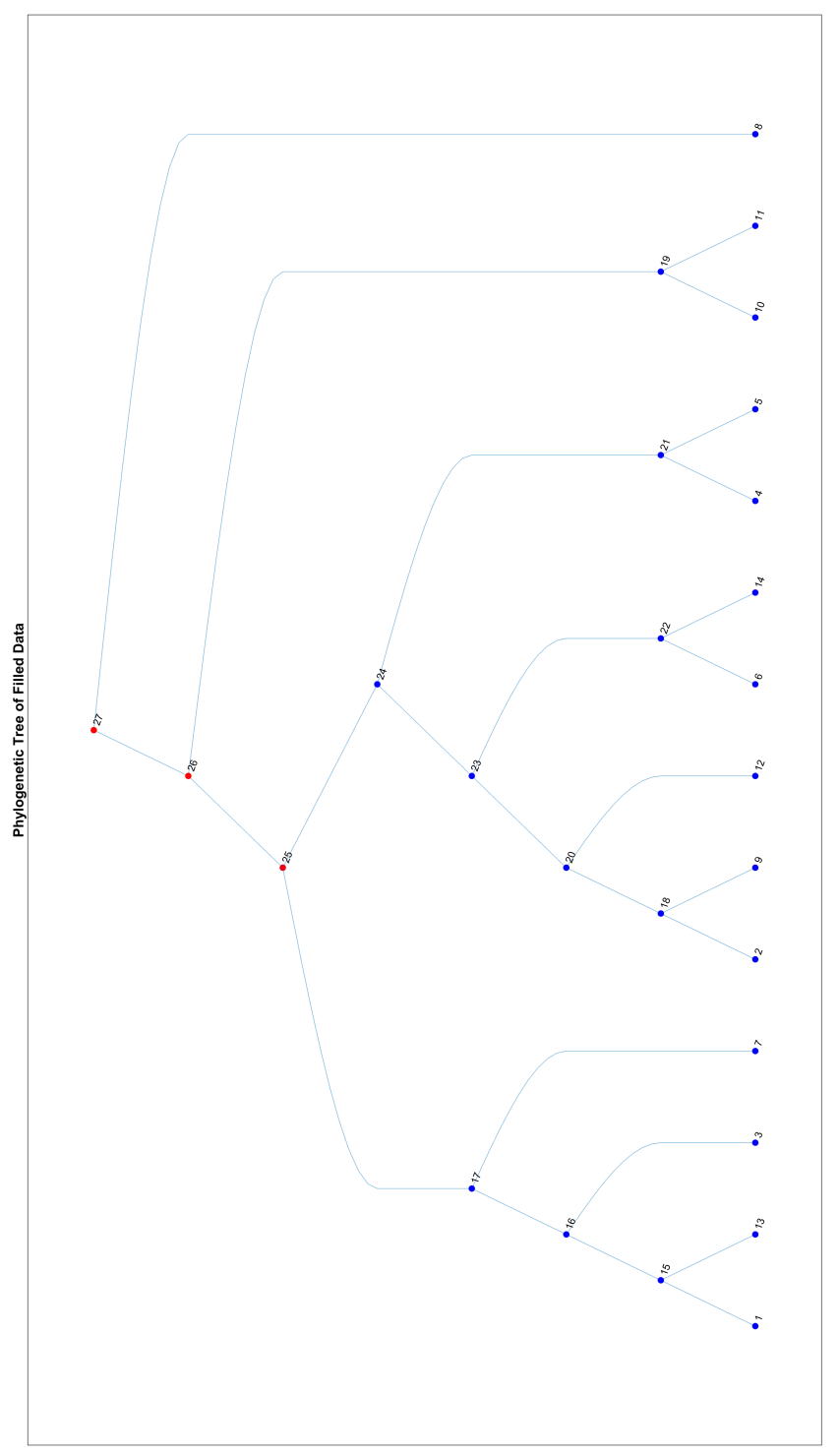

Figure 27. Persistent components tree of Germanic languages from filtered SSWL data, PCA $60 \%$. 

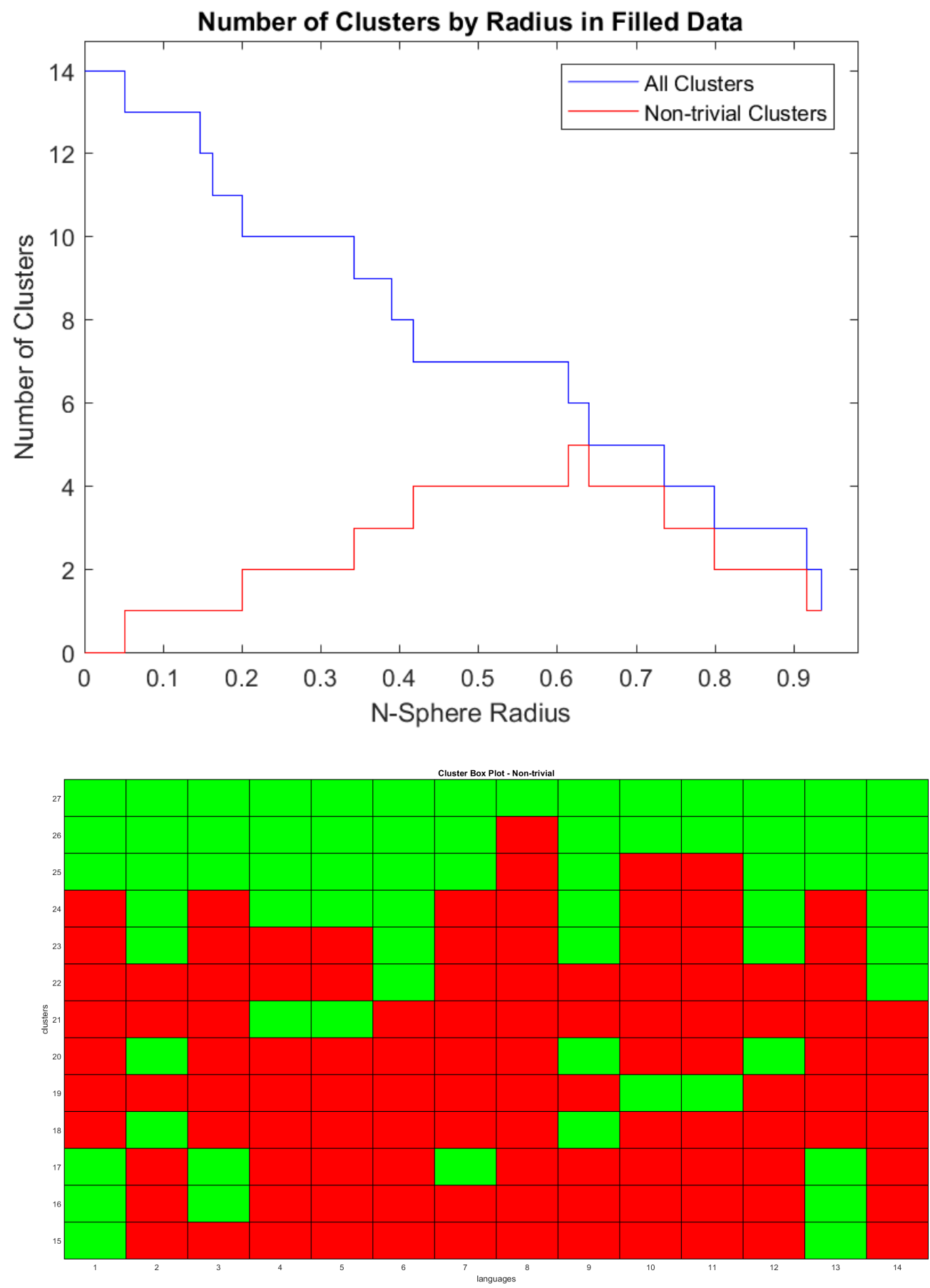

FiguRE 28. Clusters and clustering structure in the persistent components tree for the Germanic languages with filtered SSWL data, PCA 60\%. 
The clustering structure (from left to right in Figure 27, see also Figure 28) has a cluster N. 17 containing Afrikaans, Dutch, German, and West Flemish which is joined to a cluster N. 24 which is in turn a merge of two subclusters: N. 23 which contains the North-Germanic languages Danish, Faroese, Norwegian, Swedish, Icelandic and cluster N. 21 which contains English and Singaporean English. Except for the fact that the English sub-cluster joins the North-Germanic sub-cluster before nerging with the West-Germanic sub-cluster, this larger cluster does show the expected West-Germanic/North-Germanic split. The ancient languages are correctly joined higher up in the tree, with one sub-cluster N.19 with Old English and Old Saxon and a singleton cluster with Gothic. In this case, the filtering and restriction to the Germanic subfamily improve the correlation of the persistent components tree to the historical phylogenetic tree.

6.4.2. The Germanic family in the LanGeLin data. The position of the Germanic languages within the tree of the connected persistent components of the LanGeLin data also presents a structure that does not entirely correspond to the expected phylogenetic tree of the Germanic languages. In this case, however, the problem does not originate from the incompleteness of the data, unlike the SSWL case: it reflects an intrinsic difference between the branching structure recorded by the persistent components and the historical phylogenetic trees. The tree obtained at PCA $60 \%$ is given in Figure 29 with the clustering structure as in Figure 30.

The clustering structure (from left to right in Figure 29p shows Gothic as a singleton joined at the root top of the whole tree, followed by a main split into two clusters of pairs, N. 9 with English and Dutch, and N. 10 with Old English and Icelandic, these two clusters merge together in cluster N. 11, which is then joined by another cluster (N. 8) consisting of Danish and Norwegian. The clustering of these two languages correctly reflects the NorthGermanic grouping. The position of Old English, English and Dutch together also correctly reflects a West-Germanic subtree, but the grouping of Icelandic together with Old English is incorrect, since Icelandic is a North-Germanic language.

6.4.3. Comparison with algebro-geometric reconstructions. The two sets (Dutch, German, English, Faroese, Icelandic, Swedish) and (Norwegian, Danish, Icelandic, German, English, Gothic, Old English) analyzed in [37] have, respectively, 90 and 68 SSWL variables that are completely mapped for all the languages in the set. The second set of languages is represented in both databases and was analyzed in [37] using separately the SSWL and the LanGeLin data.

When we restrict to one of these small sets of Germanic languages analyzed in [37, the set (Norwegian, Danish, Icelandic, German, English, Gothic, Old English) which includes two ancient languages, we find that at PCA variance level $60 \%$ we obtain the tree

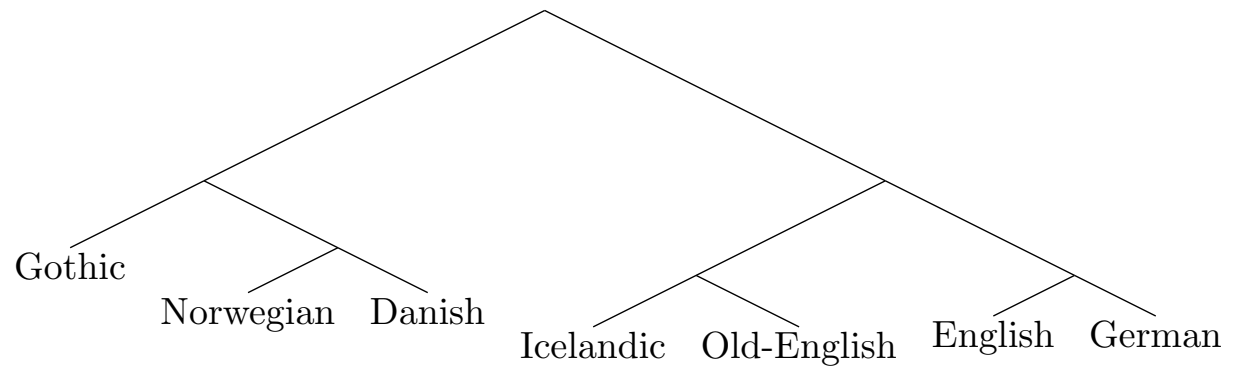




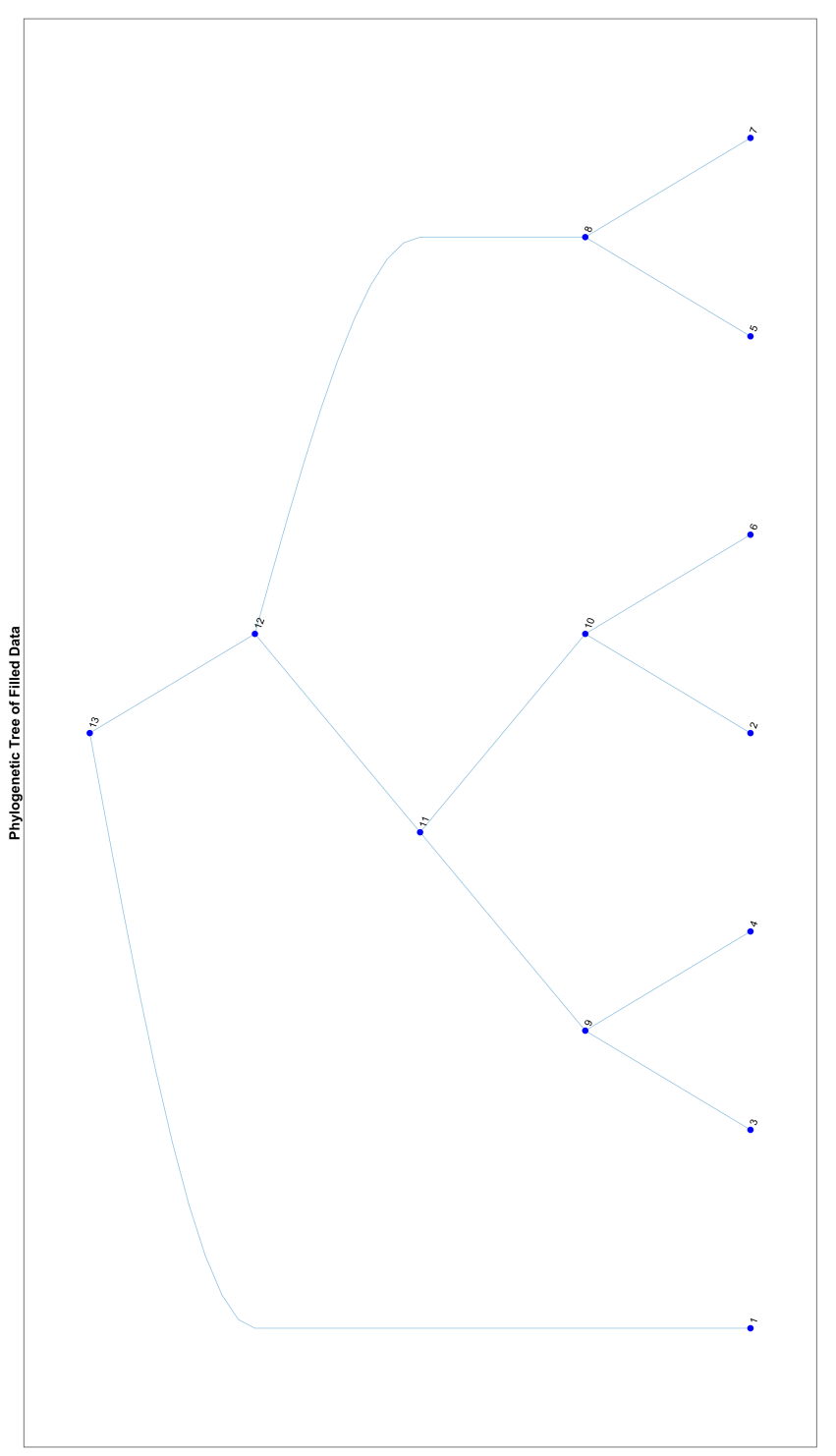

Figure 29. Persistent components tree of Germanic languages from LanGeLin data, PCA $60 \%$. 

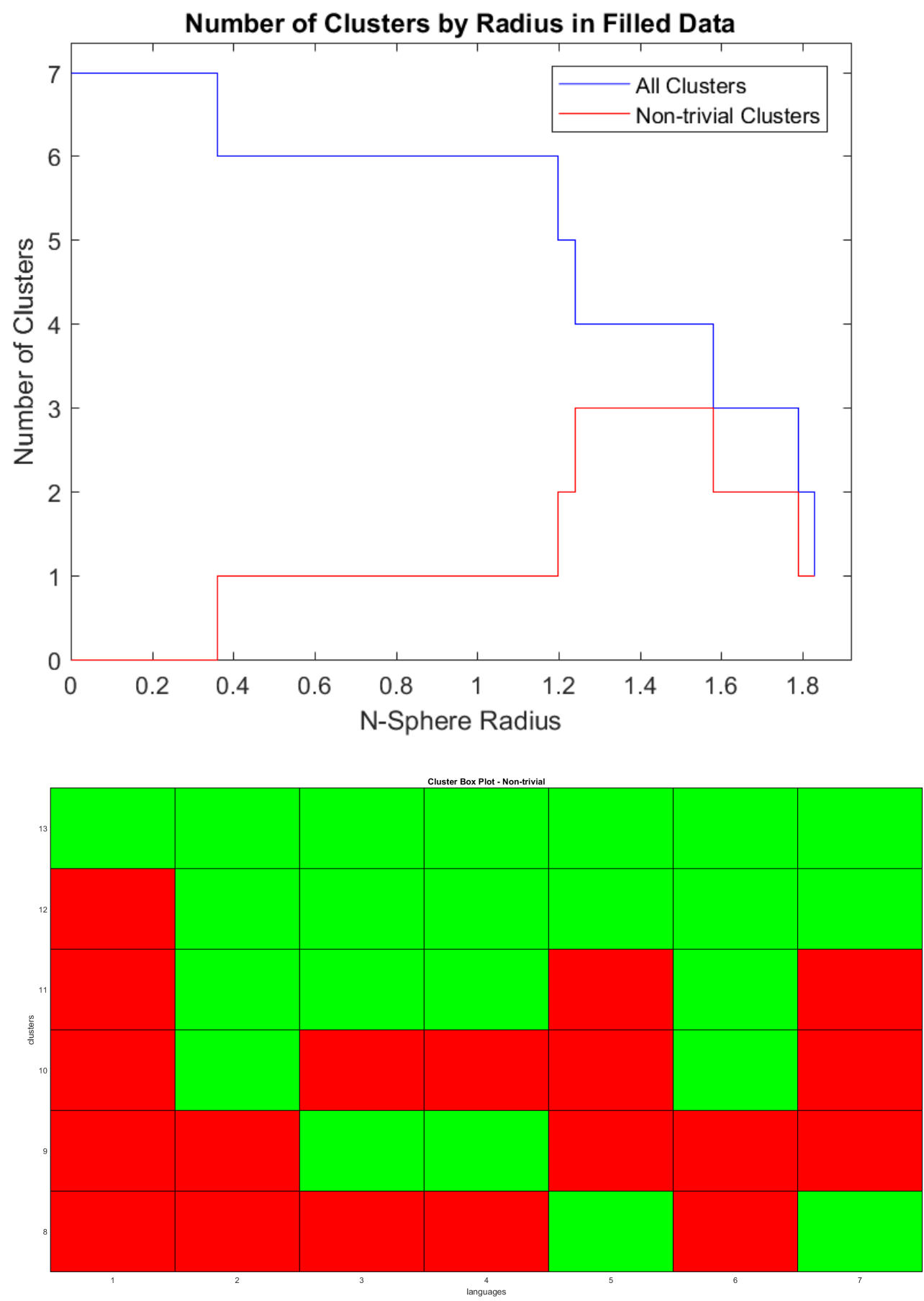

Figure 30. Clusters and clustering structure in the persistent components tree for the Germanic languages with LanGeLin data, PCA $60 \%$. 
which does not correspond to the historical tree. At PCA variance level $80 \%$ we obtain a different tree

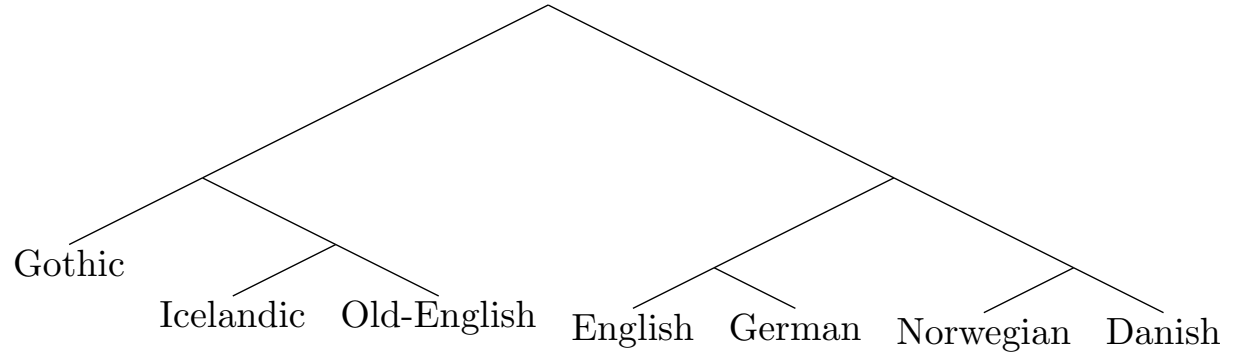

which is still not corresponding to the historical tree, but which appears as one of the candidate phylogenetic trees produced by PHYLIP, the candidate $T_{5}(G)$, in [37]. The correct phylogenetic tree, which is also identified correctly by the phylogenetic algebraic geometry method of [37], is given by

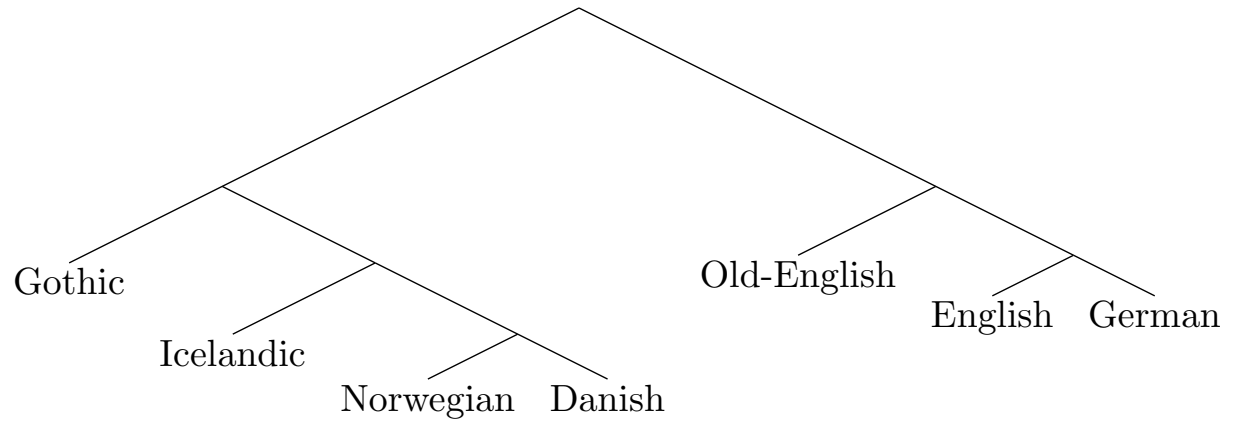

Thus, we see that, unlike in the setting of phylogenetic reconstructions discussed in [39] and in [37, the persistent component trees continue to differ from the phylogenetic trees even when only fully mapped syntactic variables are considered, with Icelandic located incorrectly in close proximity to Old-English and the grouping of languages not reflecting the North/West Germanic split.

Moreover, this example shows that the effect of the PCA variance level on the clustering of the persistent connected components has a detectable effect in the altering the tree topology. It also shows a clear case where the clustering structure of the persistent connected components does not necessarily reflect the phylogenetic tree considered to be the correct one by historical linguistics.

The algebro-geometric method of [37] yields the correct phylogenetic tree for the Germanic languages, with both the SSWL data (when only completely filled variables are retained) and for the LanGeLin data, with the correct subdivision into the North-Germanic and the West-Germanic branches and the position of each language within the branches is correct. In our case, when considering the other set of Germanic languages (Dutch, German, English, Icelandic, Faroese, Swedish) discussed in [37] we correctly get that the closest languages to each other are Faroese to Icelandic and Dutch to German, but not only that we get Swedish (incorrectly) a West Germanic language, but it also occurs as the farthest language (out of the ones in this list) from Faroese and Icelandic, which is also incorrect.

6.5. Romance language family. The second subfamily of the Indo-European family that we analyze more closely is the Romance languages. This subfamily contains all the modern Indo-European languages that have historically developed from Latin. We find that the 
LanGeLin data, that includes a set of Southern Italian dialects that had historically interesting interactions with the Greek language family, has a persistent components tree that separates out this cluster of dialects from the main Romance languages (French, Italian, Spanish, Portuguese, Romanian). The persistent components tree of the main Romance languages does not entirely match the phylogenetic tree. When restricted to the part of the tree that contains only these main Romance languages and using data filtered by completeness, the tree obtained from the SSWL data agrees with the one obtained from the LanGeLin data and differs from the historical phylogenetic tree by having the positions of Spanish and Italian flipped.

6.5.1. Romance language family in the SSWL data. The Romance languages are modern languages that evolved from Latin. The family is represented in the SSWL database by Bellinzonese, Calabrian, Catalan, French, Galician, Italian, various Italian dialects (Napoletano Antico, Old, Reggiano), Latin, Late Latin, Neapolitan, Occitan, Old French, Brazilian Portuguese, European Portuguese, Romanian, Sicilian, Spanish, Teramano.

When we consider the full tree of the persistent components for the unfiltered SSWL data, most of the Romance languages are indeed grouped together in a subtree (which corresponds to Cluster 125). This contains most of the Romance languages, including Italian, Portuguese, Spanish, Catalan, Sicilian. It also contains misplaced languages, such as English that is incorrectly placed outside of the German language family.

Looking at this subtree we notice that, although it contains many of the Romance languages, it notably does not contain French and Romanian. The languages French and Old French are quite close to each other in the tree but are added later as singletons and are very far from the rest of the Romance languages. Romanian on the other hand is not as far from this subtree as French is, but is not close either. Another thing to note is that Romanian is clustered with European-Portuguese, which we would also expect to see together with the other Romance languages. It is known that Romanian shares grammatical features with non-Romance languages such as Greek, Bulgarian and Serbo-Croatian, and it is indeed placed closer to these languages in our tree. Going back to the subtree 125 we also note that topology-wise the languages are not located accurately, as we would expect, for example, to have Spanish and Portuguese in closest proximity as Iberico-Romance languages.

Thus, we encounter once again the same kind of problems illustrated in [39] when using the full unfiltered SSWL data.

We then consider only the subset of Romance languages that are at least $50 \%$ complete in the SSWL database and we select only the subset of SSWL syntactic variables that are fully mapped for all of those languages. We analyze again the persistent components when using only this set of Romance languages and only their subset of completely mapped parameters.

The resulting tree (Figure 31) and clustering structure (Figure 32) has several interesting sub-structures. The clusters (from left to right in Figure 31) show as sub-cluster joining at the root top of the tree the correct placement of the ancient languages: cluster N. 26 containing Latin and Late Latin. This is followed by three singleton clusters, that also join near the root of the tree (in descending order after the Latin cluster): N. 8 Romanian, N. 2 Northern Calabrian, N. 14 Old French. There is then a large structure (cluster N. 22) consisting of several sub-structures. Two two-language clusters, N. 16 with Italian 


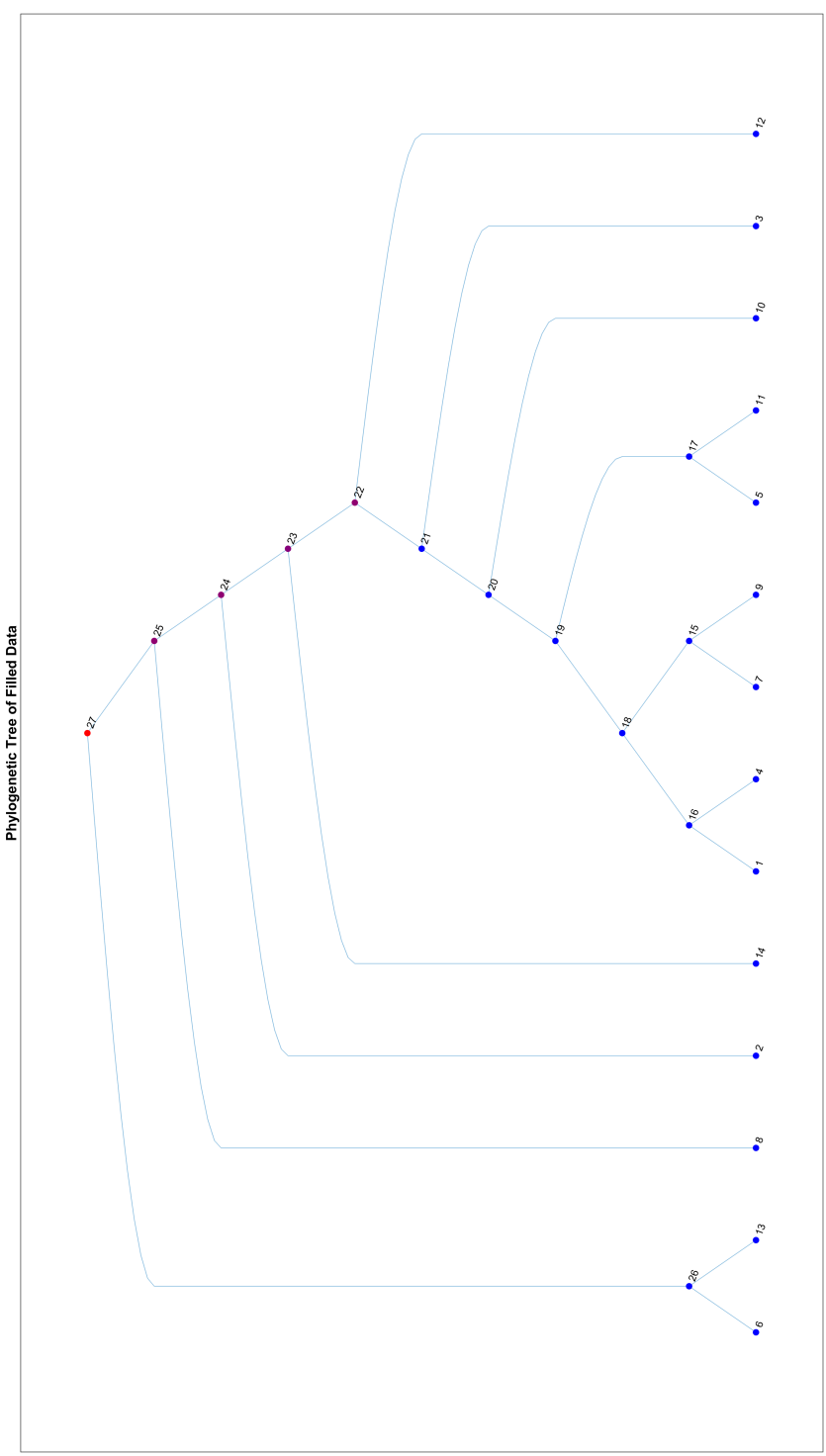

Figure 31. Persistent components tree of Romance languages from filtered SSWL data, PCA $60 \%$. 

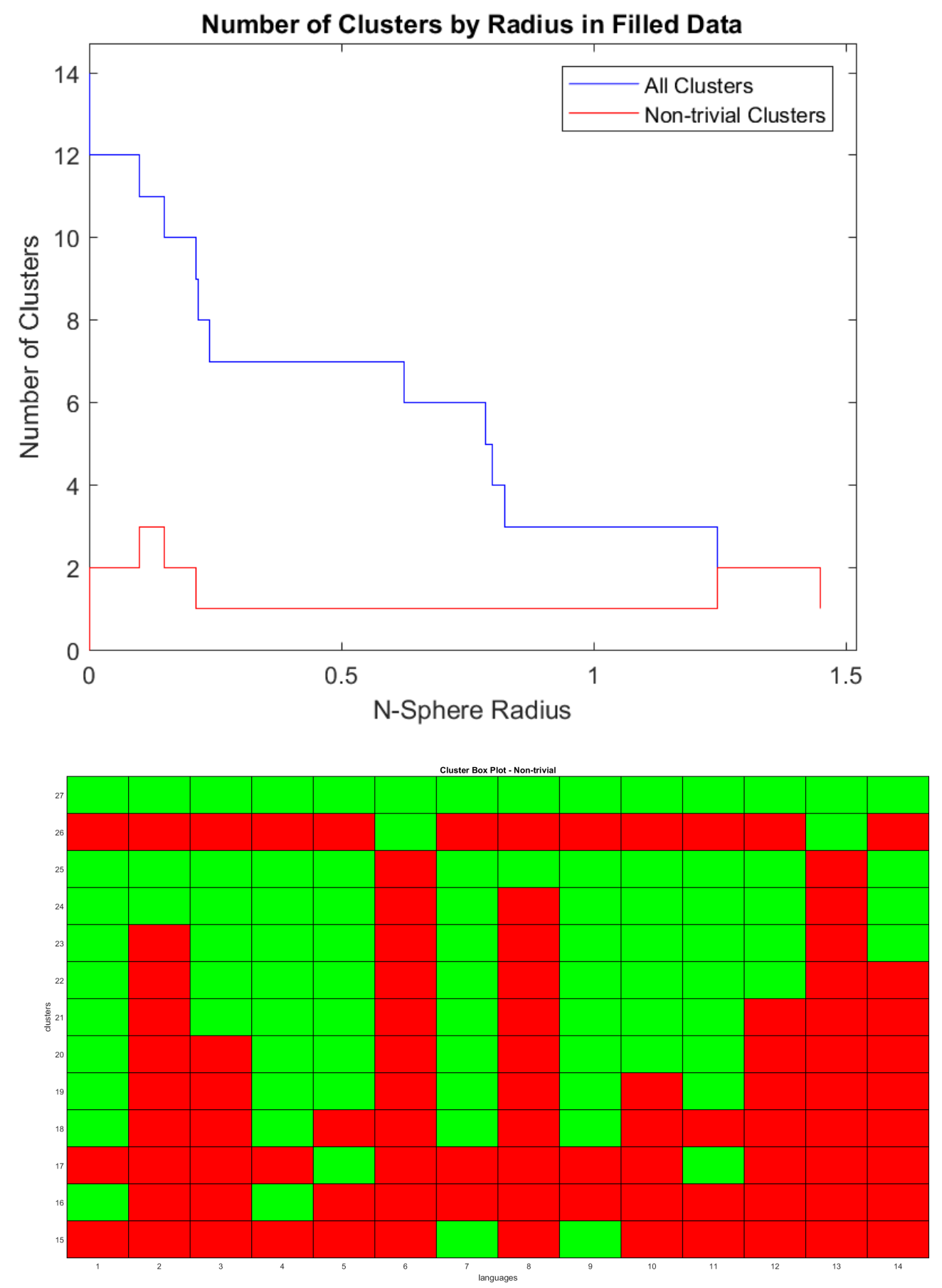

Figure 32. Clusters and clustering structure in the persistent components tree for the Romance languages with filtered SSWL data, PCA $60 \%$. 
and Brazilian Portuguese and N. 15 with Sicilian and Portuguese are joined together into cluster N. 18. This cluster then merges with another two-language cluster, N. 17 containing French and Napoletano Antico, followed by two singleton clusters, N. 10 Spanish and N. 3 Catalan. The clustering of Italian and the Southern Italian dialects together with Brazilian Portuguese, Portuguese, and French, respectively appears at odds with historical phylogenetic trees and the position of the main Romance languages also does not match the position expected in the phylogenetic tree as we discuss in Section 6.5.3.

6.5.2. Romance family in the LanGeLin data. In the case of the Romance languages, the LanGeLin data deliver a persistent components tree that is more informative and accurate in terms of reflecting phylogenetic information than in the case of the Germanic languages discussed above.

The Romance languages represented in the LanGeLin data include Latin, the main modern Romance languages French, Spanish, Italian, Portuguese, Romanian, and a set of Sourthern Italian dialects that were studied in [13] in relation to the Greek-Italian microvariations that we already mentioned. This group of Sourthern Italian dialects is given by Ragusa, Mussomeli, Aidone, Northern Calabrese, Southern Calabrese, Salentino, and Campano. When we compute the persistent components tree for the Romance languages in the LanGeLin data, the PCA at $60 \%$, we find the structure illustrated in Figure 33 . The tree illustrated in the figure corresponds to the structure of cluster N. 23. It contains a subcluster N. 12, which consists of Latin alone, and which should in fact really correspond to the root of the tree, and two main subclusters, N. 20 and N. 21. The content of the two subclusters is illustrated in Figure 33. we see that cluster N. 20 contains all the Southern Italian dialects, while cluster N. 21 contains all the main Romance languages. form

The persistent components tree for the cluster of the Southern Italian dialects has the

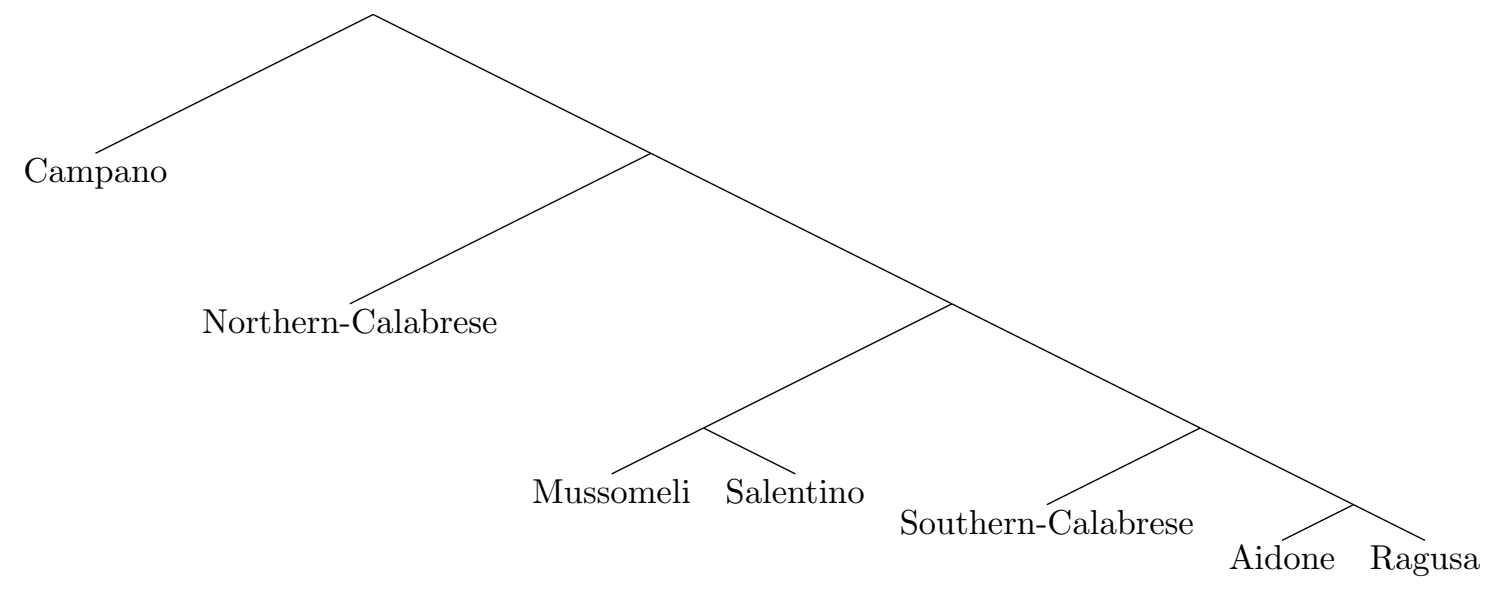

6.5.3. Comparison with the algebro-geometric method. In [37] the subset of Romance languages (Latin, Romanian, French, Spanish, Portuguese) is analyzed with the algebrogeometric method using a combination of the Longobardi and the SSWL databases, where in the SSWL only the parameters that were completely mapped were retained, and with the additional information that Latin should be regarded as the root vertex.

The topology of the tree obtained in [37] reflects the historical phylogenetic tree and is of the form 


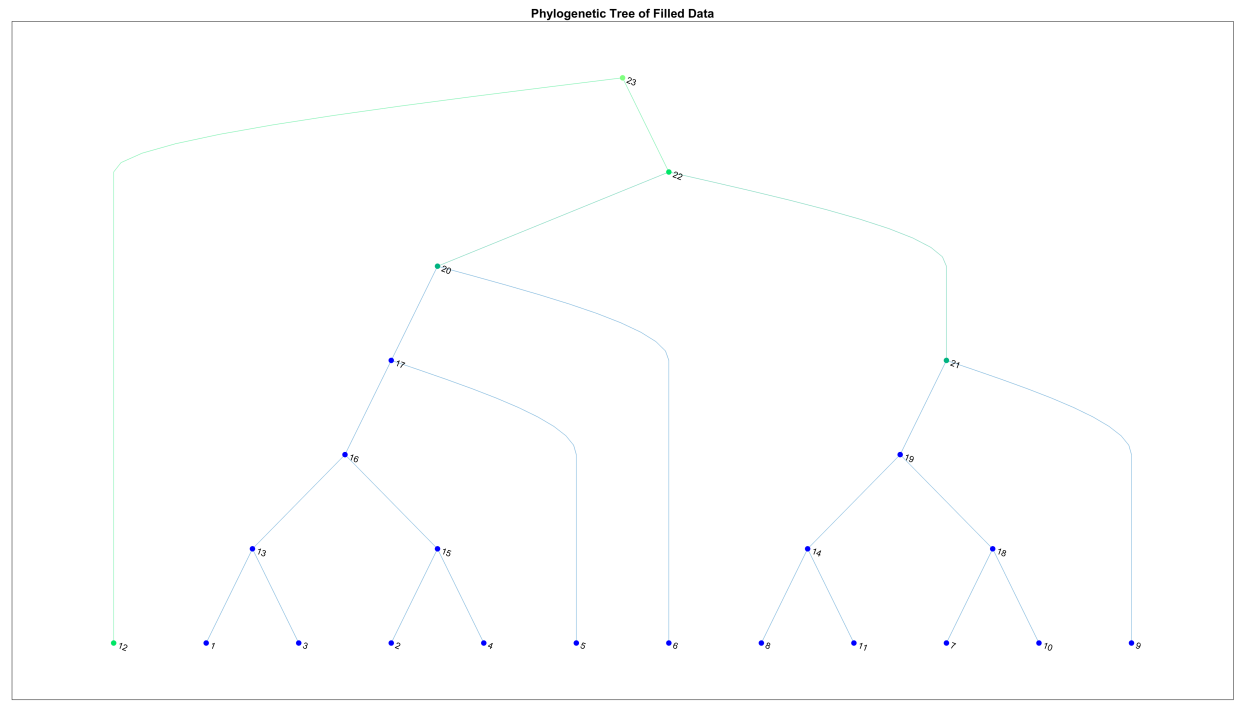

(A) Romance languages tree

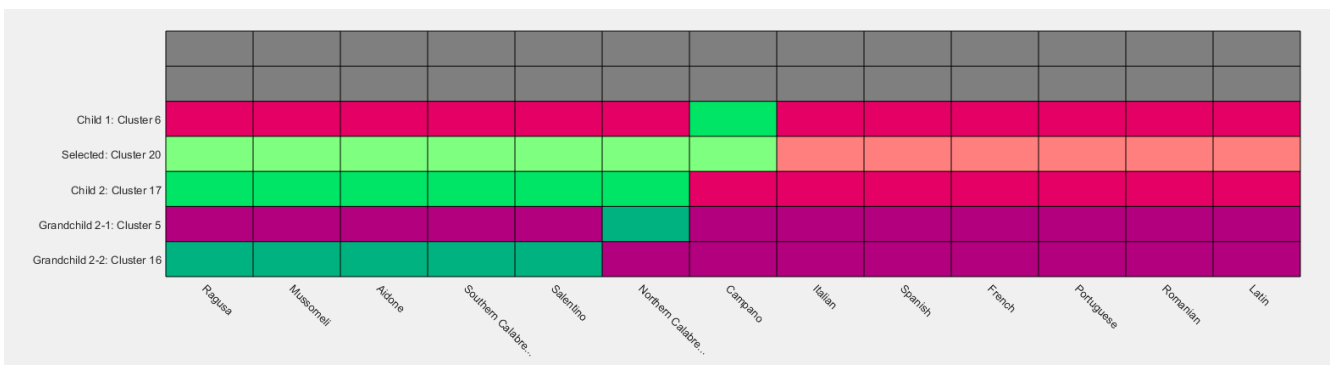

(B) Cluster N. 20

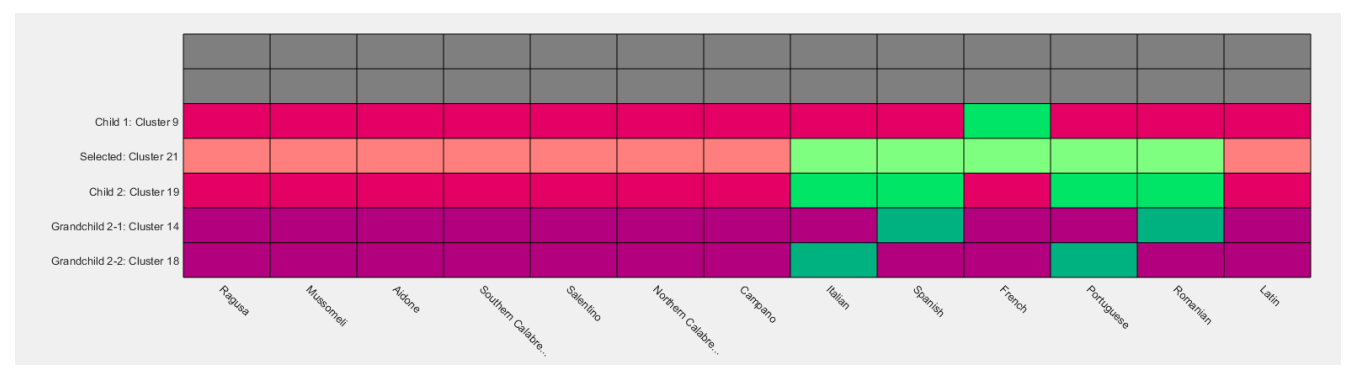

(C) Cluster N. 21

Figure 33. Persistent components trees of the Romance languages from the LanGeLin data with PCA $60 \%$. 


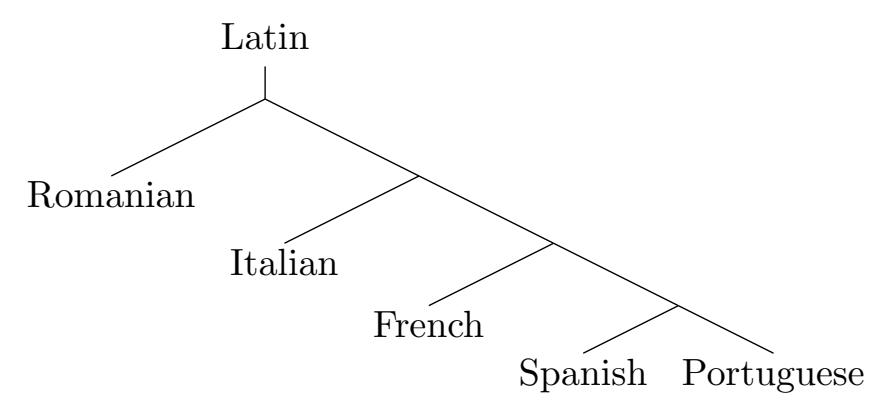

The persistent component tree for the LanGeLin data analyzed above gives a tree with the inverted position of Italian and Spanish

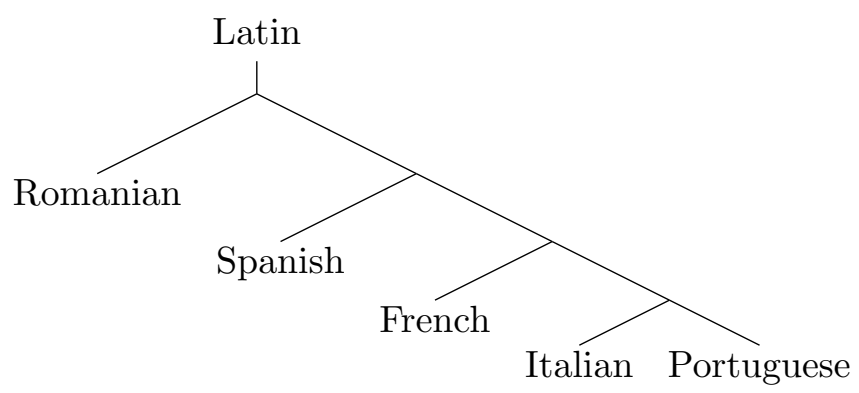

It is interesting to notice that the tree topology obtained from the filtered SSWL data, when restricted only to the main Romance languages (Latin, Romanian, Italian, French, Spanish, Portuguese) is also of the same form as the one obtained from the LanGeLin data, with the positions of Italian and Spanish inverted with respect to the historical phylogenetic tree.

6.6. Hellenic language family. The Hellenic language family in the SSWL, after filtering the data for completeness, only consists of Ancient Greek, Homeric Greek, Medieval Greek, Modern Greek, Cappadocian Greek, and Cypriot Greek. The associated tree has topology

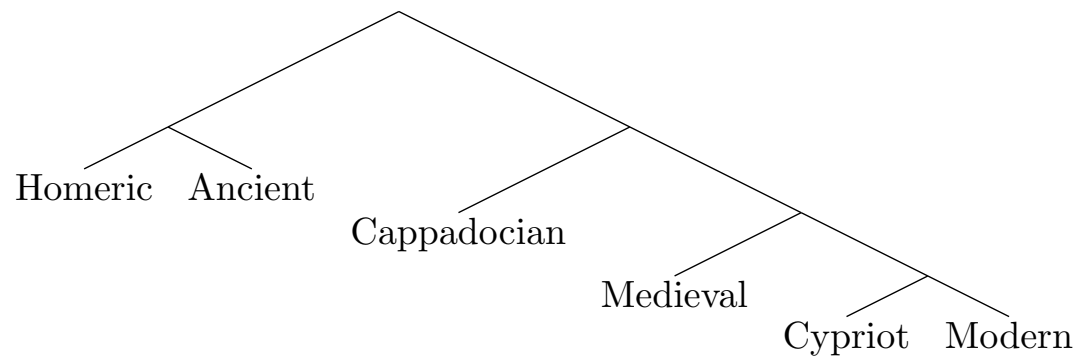

The Hellenic family in the LanGeLin data is rich with the additional presence of the Greek Southern-Italian dialects. The resulting tree topology is given by 


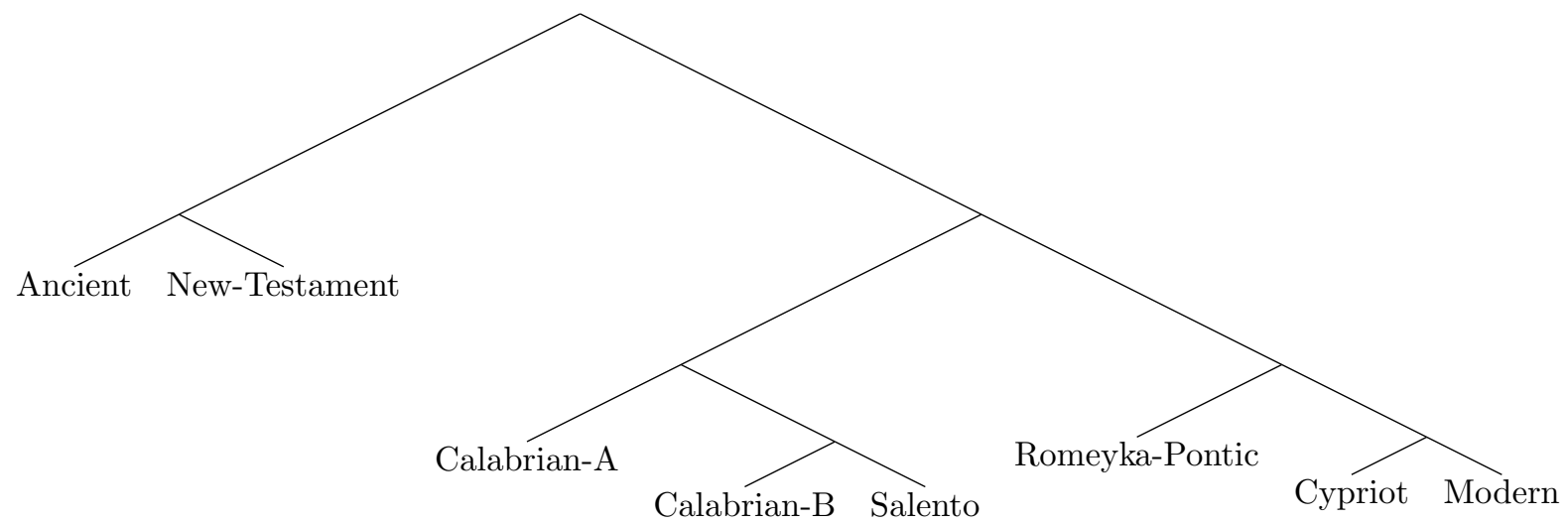

6.7. Balto-Slavic language family. The Balto-Slavic language family is comprised by two main branches: the Baltic languages, such as Lithuanian and Latvian, and the Slavic languages. In the SSWL database the Balto-Slavic languages include Bulgarian, Croatian, Czech, Lithuanian, Polish, Russian, Serbian, Slovenian, Ukrainian.

6.7.1. Balto-Slavic languages in the SSWL data. When we work with the full unfiltered set of SSWL data the Balto-Slavic language family does not demonstrate a special structure and the Balto-Slavic languages are not even grouped together. Polish and Russian are added as singletons and are far away from the rest of the languages within the tree, and from each other. Two Slavic languages that are grouped together (but not in the closest proximity) are Serbian (West Slavic) and Bulgarian (East Slavic) and are placed closer to Lithuanian more than to Polish and Russian. There aren't enough languages in this group left after filtering the SSWL data to apply the previous technique.

6.7.2. Balto-Slavic family in the LanGeLin data. The set of Slavic languages included in the LanGeLin data is given by Bulgarian (Blg), Serb-Croatian (SC), Slovenian (Slo), Polish (Po), Russian (Rus). As we already observed before, in the tree obtained from the persistent connected components we find the Slavic languages grouped together near the Germanic languages, in the same sub-cluster structure that also contains the Greek languages and Latin. An interesting phenomenon one observes in this cluster is the fact that Bulgarian is not adjacent to the other Slavic languages but is placed in proximity to the Greek languages. The resulting tree structure (drawn without resolving the substructures corresponding to the Germanic and Greek languages) has the shape

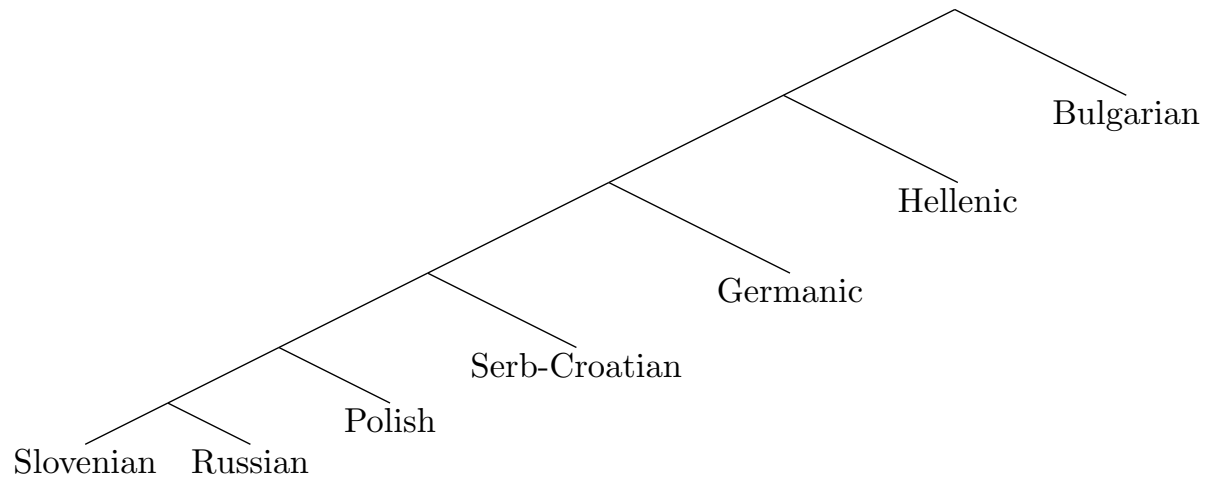


This structure appears to suggest the presence of a loop involving the Slavic and the Greek languages (possibly involving some of the Germanic languages). The presence of a loop involving the Hellenic branch and some of the Slavic languages was already observed in [35. (based on the analysis of a cluster in the SSWL data). We will discuss more in detail the $H_{1}$-structures in Section 7 and in particular a persistent $H_{1}$-generator involving some Greek and Slavic languages and Gothic, which may be another manifestation of the same linguistic phenomenon of syntactic relatedness.

6.7.3. Comparison with the algebro-geometric method. In [37] the set of Slavic languages (Polish, Russian, Bulgarian, Serb-Croatian, Slovenian) is analyzed using the combined LanGeLin and SSWL data (retaining only completely mapped variables). The phylogenetic algebraic geometry method correctly identifies the phylogenetic tree for this set of languages according to historical linguistics, given by

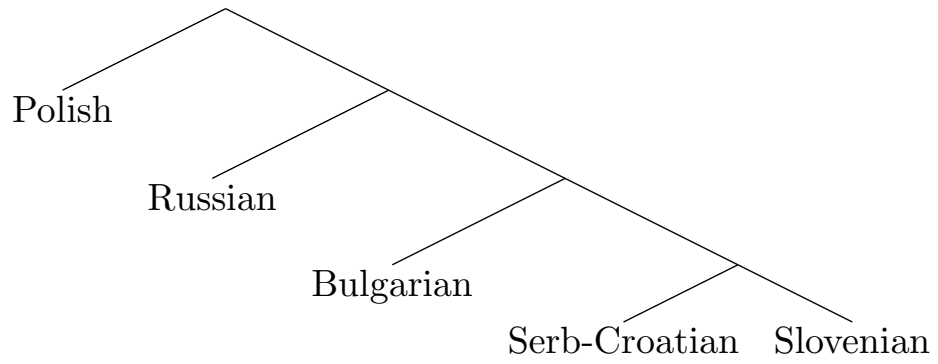

By isolating the Slavic languages in the LanGeLin data persistent components tree discussed above we find a tree with topology

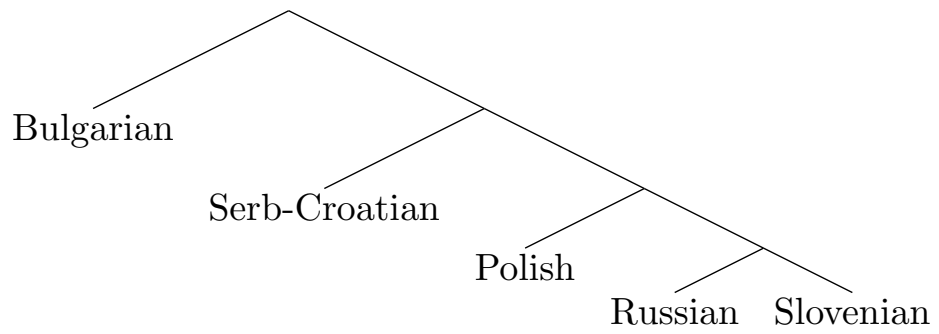

This is not reflecting the phylogenetic structure of the Slavic languages, nor does it reflect the correct placement of Bulgarian in the South-Slavic subbranch. Thus, this is another example where one sees that the information on syntactic relatedness captured by the persistent connected components tree is not the same as the phylogenetic information about historical language development, although it correlates to it in terms of grouping together (most of) the languages within the subfamily.

6.8. The hypothetical Ural-Altaic family. The LanGeLin data include the Uralic languages Estonian, Finnish, Hungarian, Udmurt, Yukaghir, Khanty and the putative Altaic languages Turkish, Buryat, Yakut, Even, Evenki, as well as Japanese and Korean that were proposed, in the early developments of the Ural-Altaic hypothesis, as other possible members of an Altaic family, although these two languages were later discarded from the Altaic hypothesis.

The persistent components tree for the LanGeLin data places Japanese and Korean in closest proximity to each other, but at the outskirts of the tree, far away from the other Altaic and Uralic languages. The rest of the Uralic and Altaic languages are all placed very closely together in cluster N. 95, with the exception of the Uralic language Yukaghir that 
is placed together with the Indo-Iranic languages. The persistent components tree for the Ural-Altaic languages in cluster N. 95 is of the form

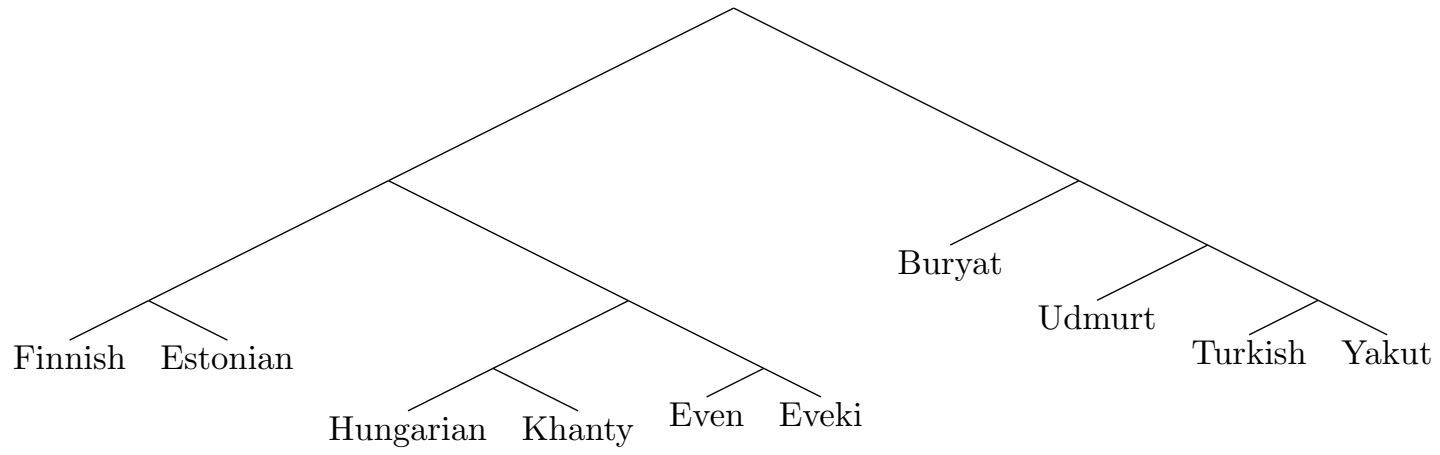

Notice that, although Udmurt is an Uralic language, it is placed in the sub-cluster with the Altaic languages, while the sub-cluster formed by the Altaic languages Even and Eveki is placed within a cluster with the Uralic languages Estonian, Finnish, Hungarian, and Khanty.

6.9. Examples from other language families in the SSWL data. An extensive representation of non-Indo-European language families is only available in the SSWL data set. In the LanGeLin data set, with the exception of the Ural-Altaic hypothesis discussed above, the non-Indo-European languages are too few to make any meaningful analysis, except for noticing the fact that they are grouped together in their own 2-leaf subtrees within the outer layer of the tree, as in the case of Arabic and Hebrew (both Semitic languages). In the SSWL database, languages in non-Indo-European families tend to be less completely mapped than the Indo-European ones. Thus, the filtering process we described above (retaining only languages that are at least $50 \%$ complete and retaining only syntactic variables that are fully mapped for those languages) tends to eliminate too many languages from these families. For this reason, we will present here the persistent components trees for these families for the full unfiltered SSWL data. These will necessarily be less reliable as they will resent from the incompleteness of the data.

6.9.1. Niger-Congo family. The Niger-Congo languages are very well represented in the SSWL database with over sixty languages (see the Introduction). When we consider the full unfiltered SSWL data, see Figure 34, the Niger-Congo tree of persistent compoments exhibits one main large cluster (N. 69), which in turn splits into two main sub-clusters (N. 62 and N. 68). Cluster N. 62 contains the languages Ewondo, Fe'efe'e, Ghomala', Kaiama Ijo, Kenyang and Koyo, while cluster N. 68 contains the languages Igala, Kindendeule, Mankanya, Medumba, Naki, Ndut. Among the smaller structures we find historically relevant clusters N. 74, which contains the languages Farefari, Gurene, Hanga, and Konni and N. 63 with Kom, Nweh, and Tukombo Tuki.

Ewondo, Fe'efe', Ghomala, Kenyang, and Koyo are all within the Southern Bantoid languages which include the Bantu group (Koyo is a Bantu C language), while Ijo (or Ijaw) is a group of putative Niger-Congo languages that are generally viewed as outside of the main branched of the Niger-Congo family. Thus, with the exception of the presence of the Kaiama Ijo language, the rest of cluster N. 62 can be seen as a Southern Bantoid structure. Within this cluster Fe'efe'e and Kenyang form the deepest sub-structure, to which Ghomala, 


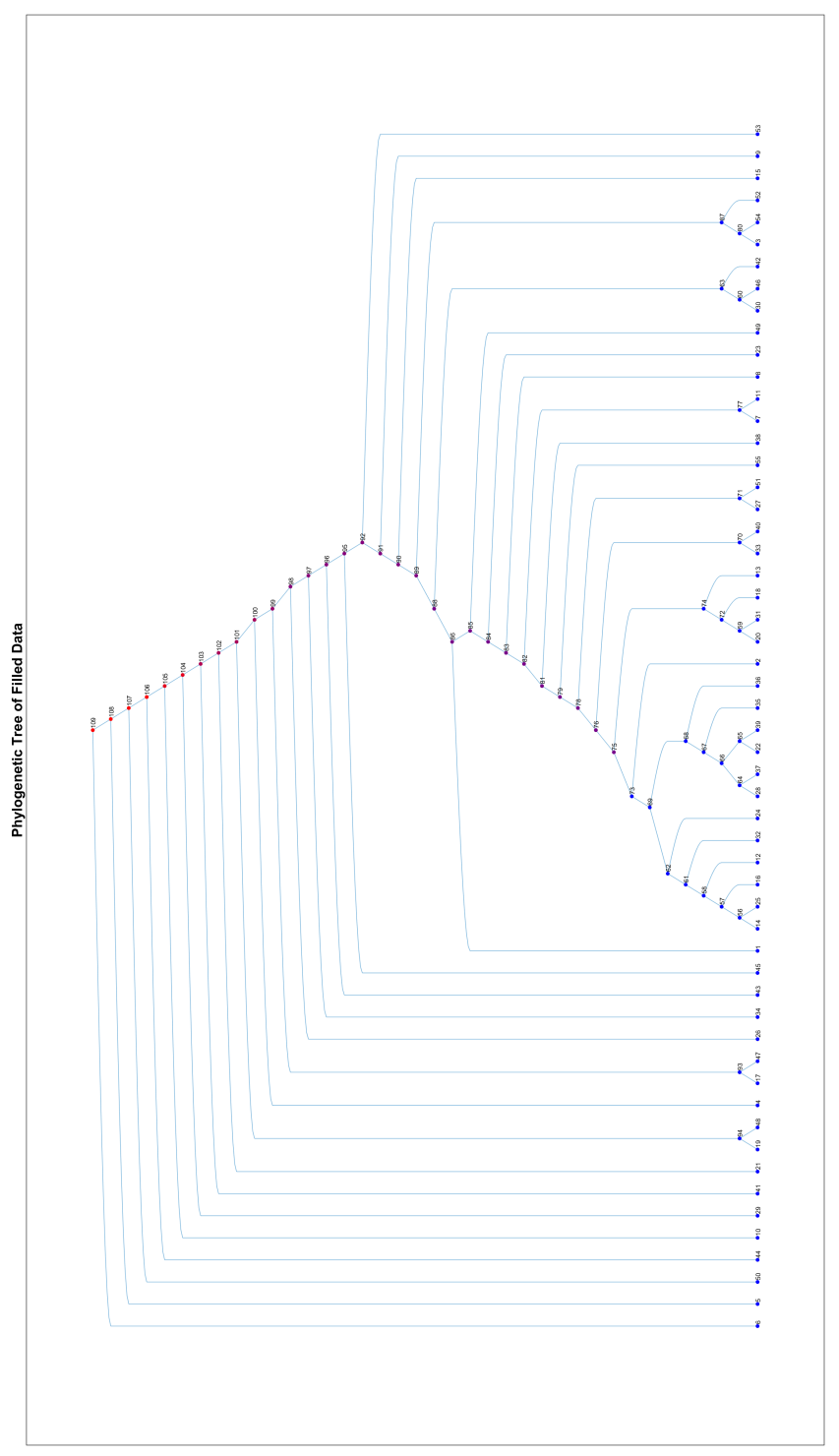

Figure 34. Persistent components tree for the Niger-Congo language family, SSWL data. 
Ewondo, Koyo, and Kaiama Ijo are successively added, with the only non-Bantoid language added last.

In cluster N. 68 we find that Mankanya belongs to the Bak group, and Ndut is a Senegambian Cangin language. Bak and Senegambian languages are considered close and often grouped together, so having Mankanya and Ndut in the same substructure is consistent with this proximity. Kindendeule is again a Southern Bantoid language, Naki is an East Beboid language, which is also part of the Southern Bantoid group, and Medumba is also Southern Bantoid (Grassfields group). The language Igala, on the other hand, is a VoltaNiger Yoruboid language, which belongs to yet another branching of the Niger-Congo languages. The Southern Bantoid family and the Bak and Senegambian groups do not belong to the same sub-branching of the Niger-Congo family: Bak and Senegambian are part of the Atlantic branch while the Southern Bantoid languages are part of the Benue-Congo group inside the Volta-Congo branch, and Igala is part of the Volta-Niger group of the Volta-Congo branch. Thus, the close proximity of the persistent connected components of these languages in cluster N. 68 does not reflect their historical relatedness. The structure within this cluster shows the pairing of Kindendeule and Naki (cluster N. 64) and Igala and Ndut (cluster N. 65): both of these mix different subfamilies of the Niger-Congo family. These two-language clusters are then joined together (cluster N. 66) and successively joined by the Bak language Mankanya and the South Bantoid language Medumba. The language Babanki, which joins just above cluster N. 69 (cluster N.73), is also a Southern Bantoid language.

Among the smaller clusters, cluster N. 74 is made entirely of Atlantic-Congo Gur languages, with Hanga and Konni placed in closest proximity, joined by Gurene and Farefari. Cluster N. 63 is made of Southern Bantoid languages, with Kom and Nweh in Grassfields group and Tuki in the Mbam group. Thus, with the exception of the mixing of different branches that occurs in cluster N. 68, the main clusters are either Southern Bantoid or Gur structures.

6.9.2. Austronesian family. The internal structure of the Austronesian languages is complex. The family consists of many similar and closely related languages with large numbers of dialect continua, making it difficult to recognize boundaries between branches and subfamilies.

The persistent components tree obtained from the SSWL data of Austronesian languages has some large structures and only four singletons added near the root of the tree (see Figure 35). The large structures consist of two main subtrees (clusters N. 32 and N. 34), with cluster N. 32 containing a main sub-cluster N. 30.

The structure of cluster N. 30 includes a pair (cluster N. 25) consisting of Palue, a Malayo-Polynesian Flores language and Kayan, a Malayo-Polynesian Borneo language. It also contains another two-language sub-cluster (N. 21) with Squliq Atayal, a Northern Formosan language, together with the Tongic Polynesian language Niuean. Both of these languages are among the most poorly mapped in this family so their occurrence together should be regarded as an accident due to the incompleteness of the data. Subcluster N. 29 of cluster N. 30 also contains Isbukun Bunun, which is a Formosan language, Ilokano (a Malayo-Polynesian Philippine language), and the Malayo-Polynesian Oceanic language Fijian. Cluster N. 32 also contains a two-language cluster with Maori (MalayoPolynesian Oceanic Eastern-Polynesian Tahitic) and Tongan (which is Malayo-Polynesian 


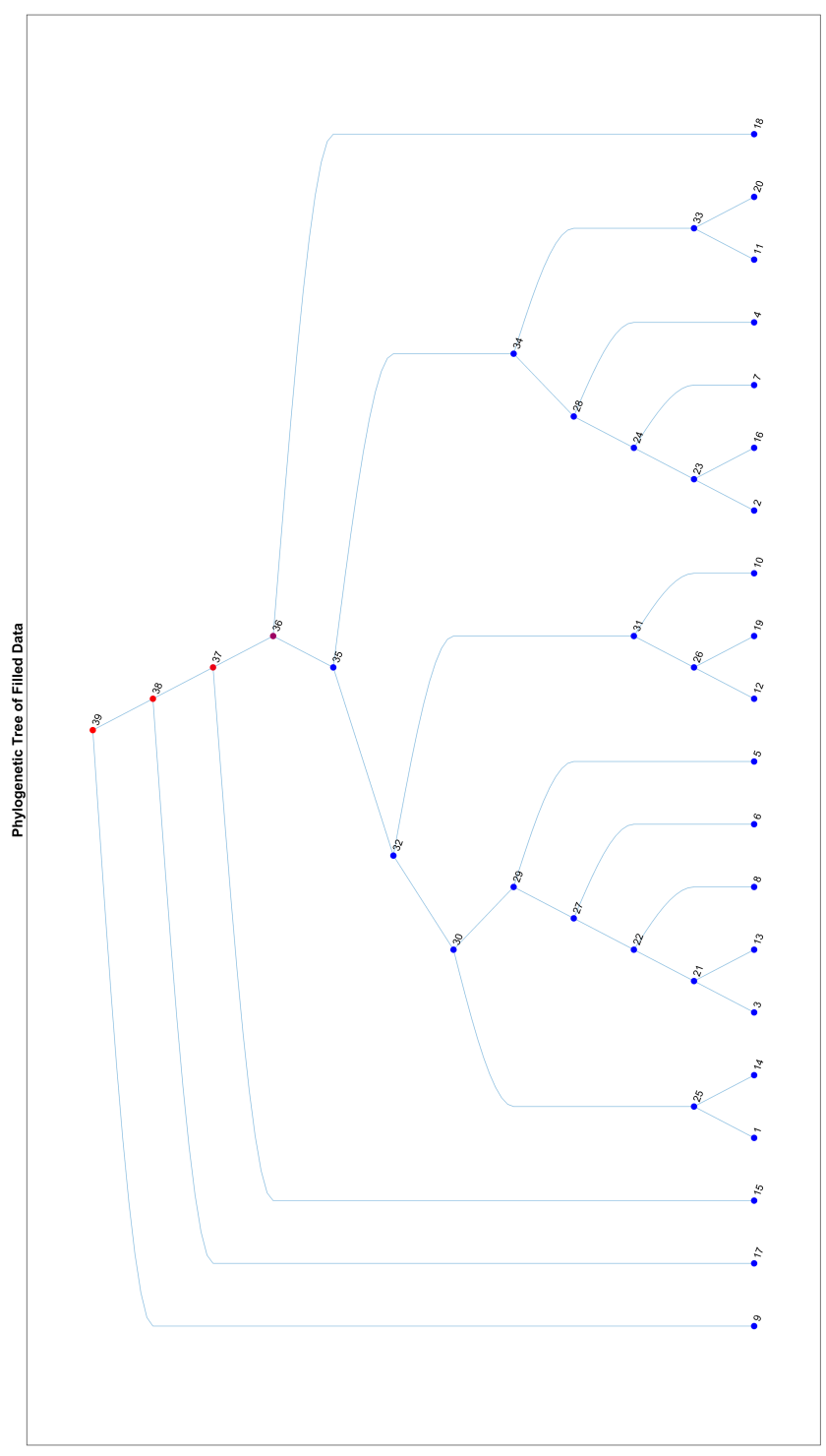

Figure 35. Persistent components tree for the Austroneasian language family, SSWL data. 
Oceanic Polynesian-Tongic). Thus, cluster N. 35 does not reflect a historical linguistic grouping, since it includes Malayo-Polynesian languages of different groups (Flores, Borneo, Philippine, Oceanic) as well as Formosan languages, but it can be seen as prevalently a Malayo-Polynesian structure.

Cluster N. 34 contains Acehnese (a Malayo-Polynesian Malayo-Sumbawan language), West Coast Bajau (a Malayo-Polynesian Borneo language), Indonesian (Malayo-Polynesian Malayic), Marshallese (Malayo-Polynesian Micronesian), Sasak (Malayo-Polynesian MalayoSumbawan), Tukang Besi (Malayo-Polynesian Celebic). Among these, the two MalayoSumbawan languages Acehnese and Sasak are grouped together in a two-language subcluster (N. 23) which agrees with historical proximity, while the two-language cluster N. 33 with Marshallese and Tukang Besi does not reflect historical proximity.

The two clusters N. 32 and N. 34 merge into cluster N. 35. The remaining singletons are Titan (a Malayo-Polynesian Oceanic Manus language) joining just above cluster N. 35 (cluster N. 36), followed by merging with Samoan (a Malayo-Polynesian Polynesian language) in cluster N. 37, with Tagalog (a Malayo-Polynesian Philippine language) and Malagasy (the Malayo-Polynesian East Barito language of Madagascar), which is the farthest away and last to merge with the rest of the tree. The structures seen in the persistent component tree of the Austronesian languages are primarily Malayo-Polynesian, but the historical linguistic subdivisions of this family into subfamilies is not preserved by the clustering of connected components.

6.9.3. Afro-Asiatic family. The persistent components tree for the Afro-Asiatic languages obtained from the SSWL data (see Figure 36) shows a subdivision into different small substructures. We find a singleton cluster N. 9 with Amharic which joins the tree very close to the root; just below comes singleton cluster N. 4 with Hebrew and singleton N. 12 with Muyang. We have then a main structure, cluster N. 24 with two sub-structures, N. 20 and N. 23 (in turn split into N. 21 and N. 22).

Cluster N. 20 has Bole, Hausa, Miya and Moroccan Arabic (with the last two grouped together). The first three are all Chadic languages, and Moroccan Arabic is placed in closer proximity to Miya of the Chadic group of Berber languages by which is historically influenced rather than together with the other Arabic languages in the Semitic languages cluster N. 18. This cluster has good correlation to a historical linguistic grouping.

Cluster N. 22 has Wolane, Tigre, and Senaya. The clustering together of Senaya and Tigre is likely an accident due to them being very incompletely mapped languages in this group. Indeed, they should not belong in the same sub-structure: Tigre is a South Semitic Ethiopic language, which is correctly placed close to Wolane and should also be close to Amharic (which occurs here as a singleton joining at the top of the tree), while Senaya is a Central Semitic Aramaic language.

Cluster N. 21 sees two language pairs, N. 17 with Biblical Hebrew and Gulf Arabic and N. 16 with Egyptian and Lebanese Arabic, merging into a cluster N. 18 of Semitic languages. Note that the fact that Hebrew occurs as a separate singleton N. 4, placed very far away from this cluster, is not an effect created by incompleteness of the data, as the level of completeness of Biblical Hebrew and Hebrew in the database is comparable and significant. It is also not an issue introduced by the PCA since the relative positions of these languages remains the same with PCA $80 \%$. The cluster N. 18 of Semitic languages is then joined 
by the Chadic language Mbuko (which is not placed among the other Chadic languages in cluster N. 20).

There are different proposals in historical linguistics regarding the phylogenetic tree of the Afro-Asiatic languages. A possible historical phylogenetic tree was discussed in [29. The only languages that are in the intersection of the Afro-Asiatic languages listed in [29] and those listed in the SSWL database are Miya, Hausa, Arabic, Lebanese-Arabic, Hebrew, Amharic, Wolane, Tigre. For this subset of languages, the phylogenetic tree proposed in 29] has the topology

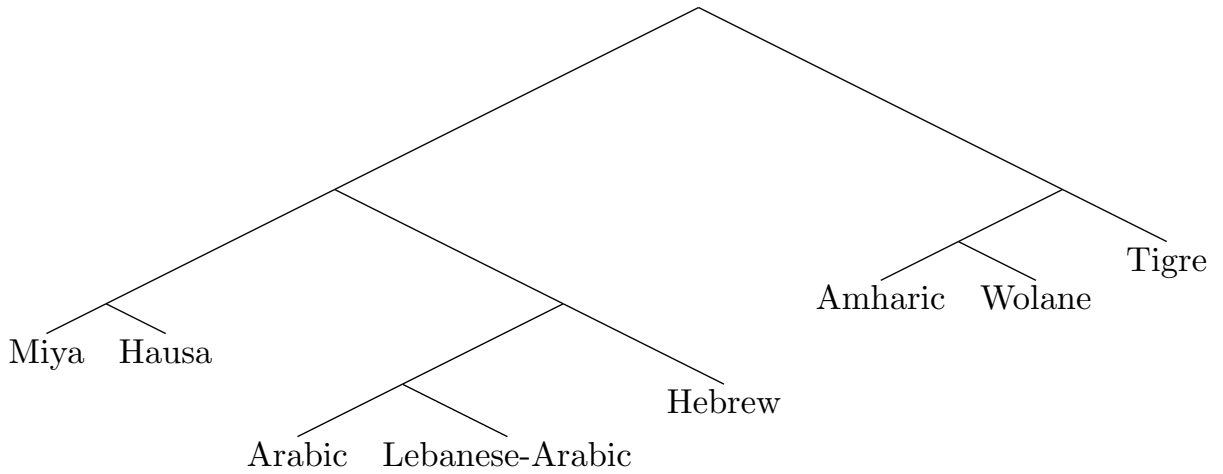

While the persistent component tree correctly places Miya and Hausa in the same substructure and Arabic, Lebanese Arabic, and Biblical Hebrew also in a substructure, it does not correctly place Amharic in the same substructure with Wolane and Tigre. Some misplacements are likely due to problems of incompleteness of the data and the poor mapping of some of the languages.

\section{Persistent First Homology}

We discuss in this section the presence of non-trivial generators of the persistent first homology $H_{1}$ for the data of languages in the SSWL and the LanGeLin database. We first observe that the behavior of persistent homology for these syntactic data is different from the typical behavior of random simplicial sets. We then discuss the method we follow for identifying specific representatives of generators of the persistent first homology and we discuss an example that appears to be detecting syntactic homoplasy phenomena as well as another example that instead may have a possible historical linguistic interpretation. We also show the barcode structure of $H_{1}$-generators for the filtered SSWL data, over individual language families. The large set of generators over the full database is more likely to be due to homoplasy detection, while $H_{1}$-generators within language families have more chances of representing possible historical influences between languages across different branches that happened at the syntactic level.

7.1. Behavior of persistent homology on random data sets. We ran the same analysis via persistent components and persistent homology computations on random data sets of binary vectors (and also for non discrete sets) with varying PCA values. The main differences are that in the random data sets $H_{1}$ appears also for smaller clusters, whereas in the case of the syntactic data from both the SSWL and the LanGeLin databases non-trivial $H_{1}$ generators are starting to appear only for bigger clusters (over the full database, for instance, they are seen only in clusters containing at least 30 different languages). Moreover, the structure of the $H_{1}$ generators themselves is different. On the random data sets 


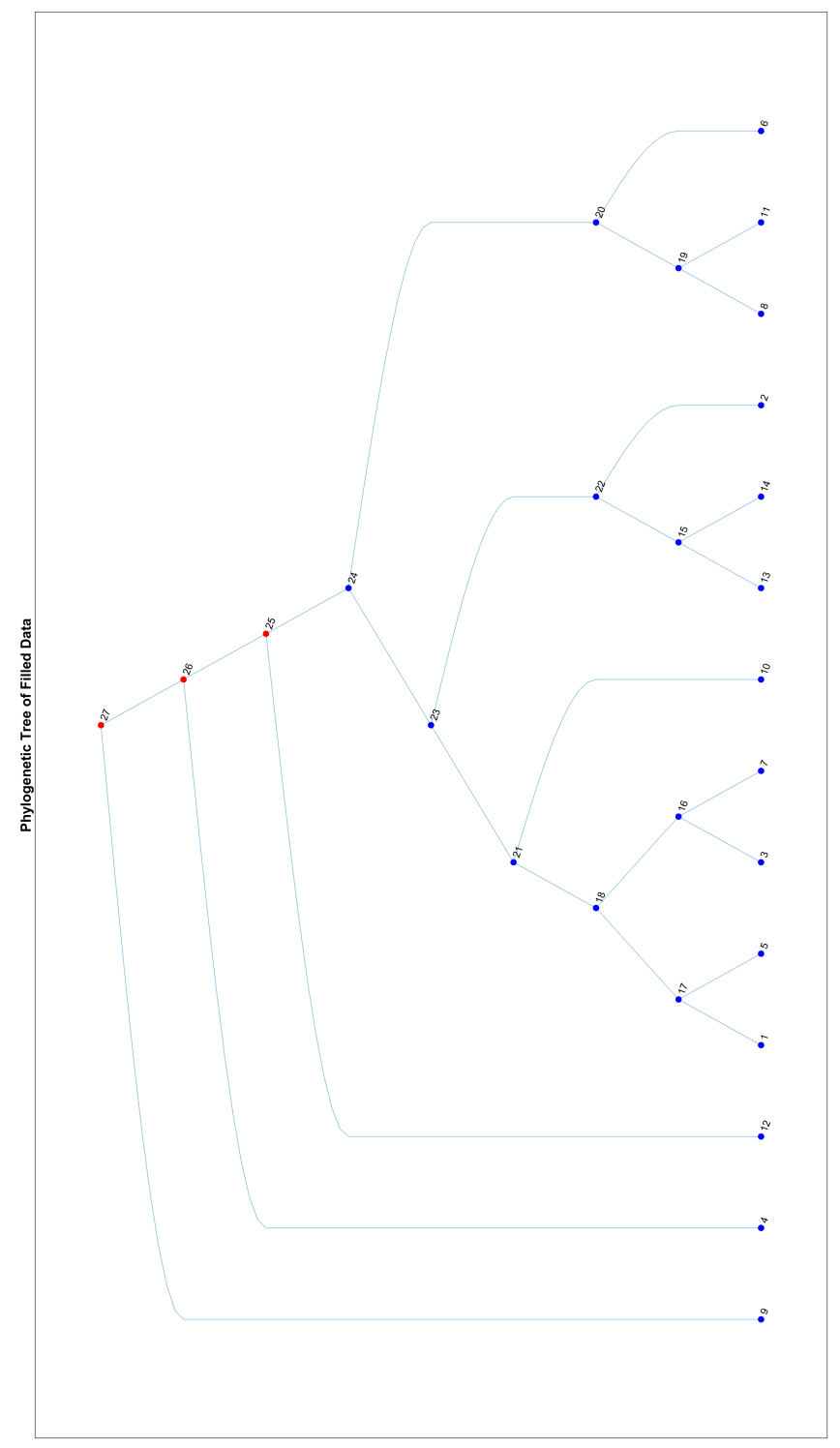

Figure 36. Persistent components tree of the Afro-Asiatic language family, SSWL data, PCA $60 \%$. 


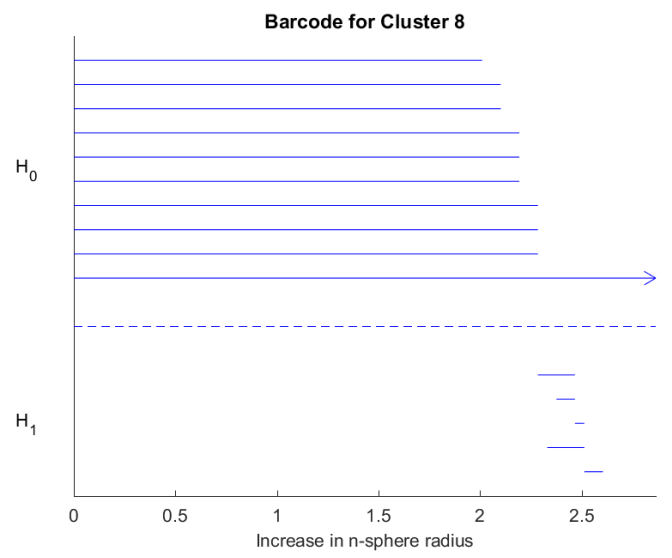

(A) random barcode

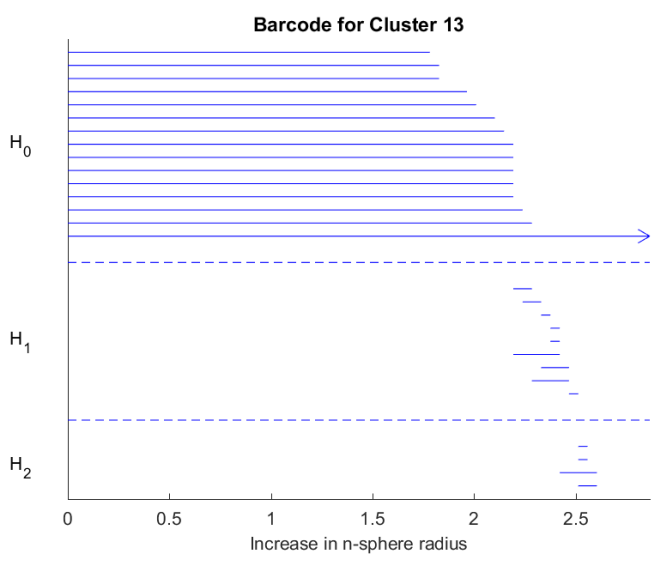

(B) random barcode

Figure 37. Barcodes of random data sets. The left barcode is of a smaller cluster and already shows several $H_{1}$-generators.

(see Figure 37) we always see that the $H_{1}$ generators are of shorter length, stacked on top of each other and less spread out. Also, there is typically a significantly larger number of generators for the $H_{1}$. On our data sets on the other hand, we also observe the presence of $H_{1}$ generators with a longer length of persistence in the barcode diagram, and they are also typically more sparse. This suggest that in our case the $H_{1}$ structure is more persistent and also less coincidental, hence more likely to reveal some genuine underlying structure in the data.

7.2. Identification of data points in $H_{1}$ generators. Along with computing the clusters via $H_{0}$ to assemble the persistent components tree, as we discussed in the previous sections. The Perseus code also computes the persistent $H_{1}$ of each cluster. In the output we only get the birth and the death of each generator of the $H_{1}$ in the form of a barcode diagram, but the persistent homology computation does not provide us with a specific choice of generators themselves for the persistent $H_{1}$ homology at each scale radius.

In particular, because no dimensional structure is detected in the data, a simple measure of the topological significance of a cluster is provided by the sum of the lengths of its persistent $H_{1}$ generators. Because of the way the tree is constructed using inclusions of clusters, this measure of topological significance monotonically increases while going up the tree from child to parent.

The natural question to ask then is when the first topologically significant cluster in a tree arises, that is, the first cluster that exhibits a non-trivial persistent $H_{1}$, and which clusters contribute to the formation of the first non-trivial $H_{1}$-generators.

In order to compute manually the generator of the significant $H_{1}$ we need to identify each group of the languages in these cycles, remove each of these language groups from our data set and compute the persistent homology again to see whether the significant structure in the $H_{1}$ still exists. The method we use to identify explicit generators of the persistent $H_{1}$ consists of the following procedure: 

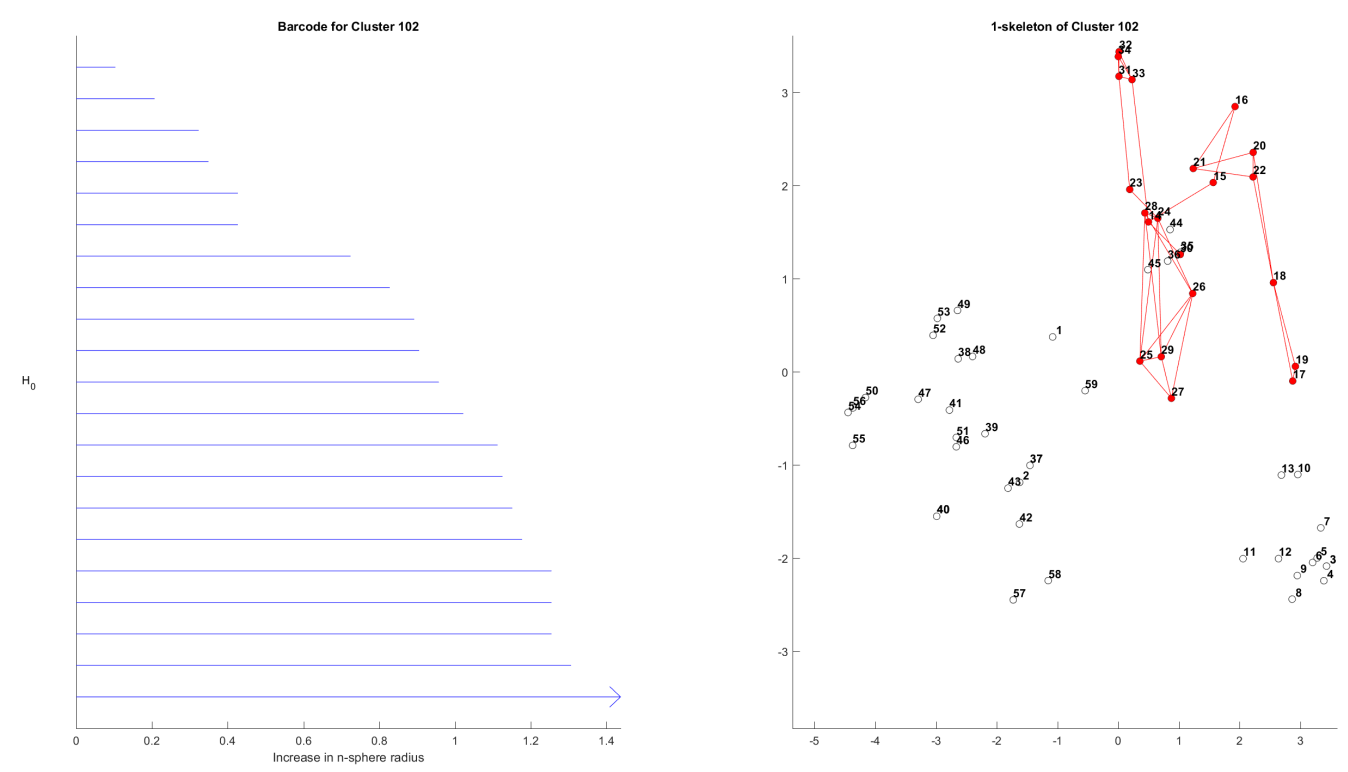

(A) Cluster N. 102
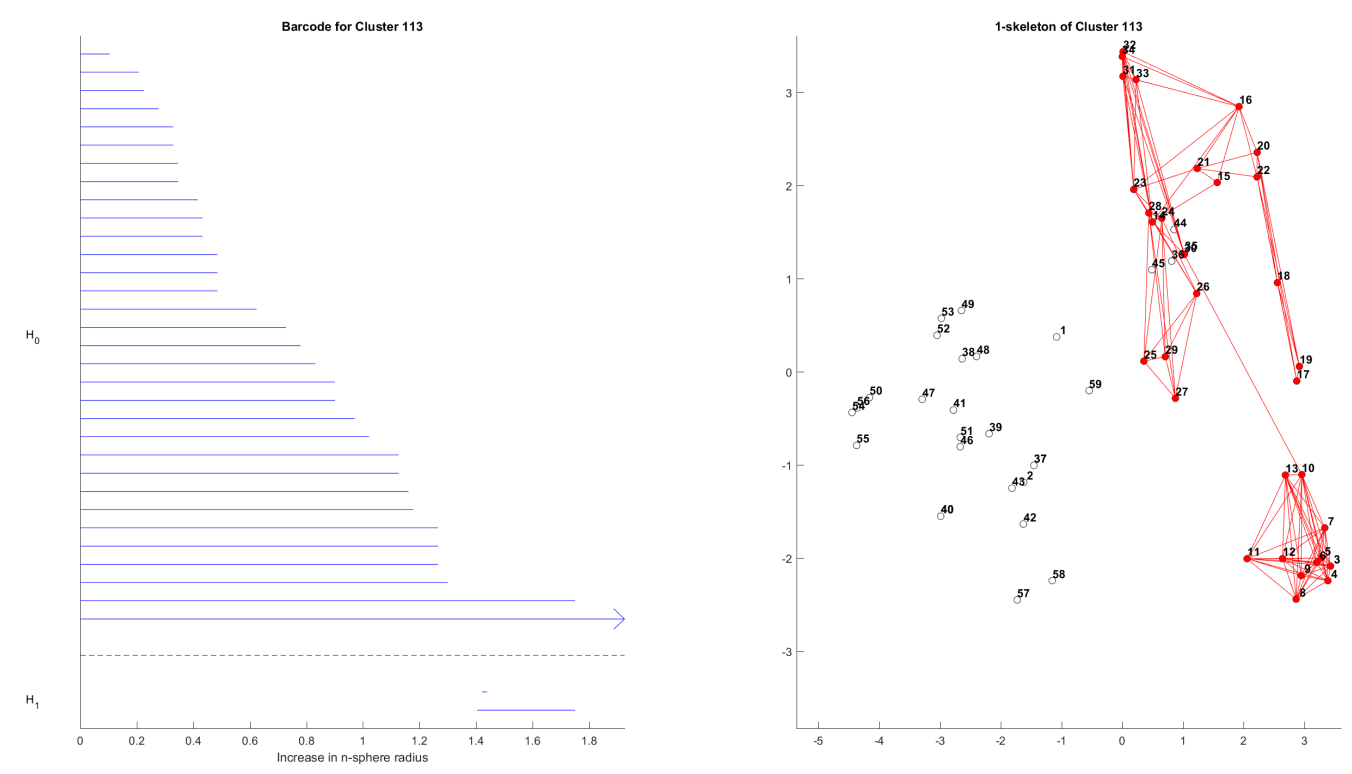

(B) Cluster N. 113

Figure 38. Formation of a non-trivial persistent $H_{1}$-generator in the IndoEuropean languages (LanGeLin data): the Gothic-Slavic-Greek loop. 

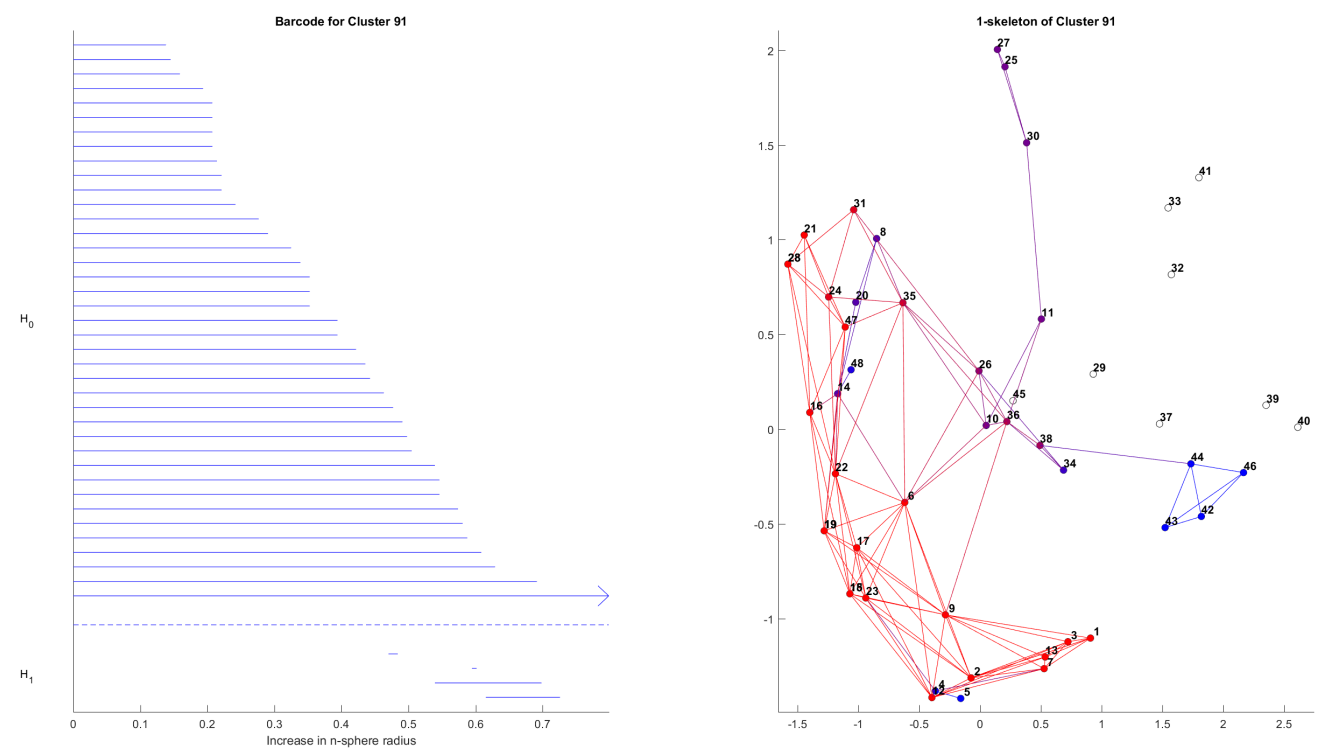

(A) Cluster N. 91
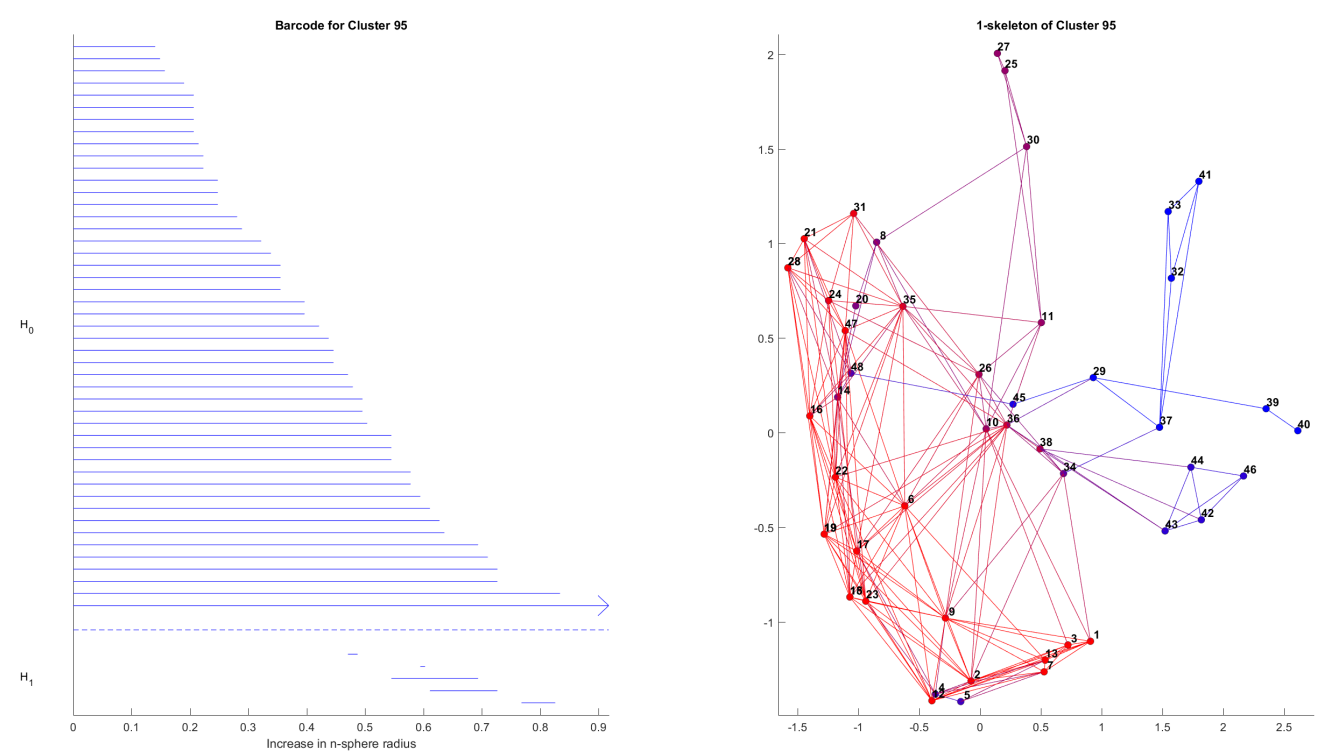

(в) Cluster N. 95

Figure 39. Persistent $H_{1}$-structures in the filtered SSWL data (including both Indo-European and Ural-Altaic languages), PCA 60\%, clusters N. 91 and N. 95. 

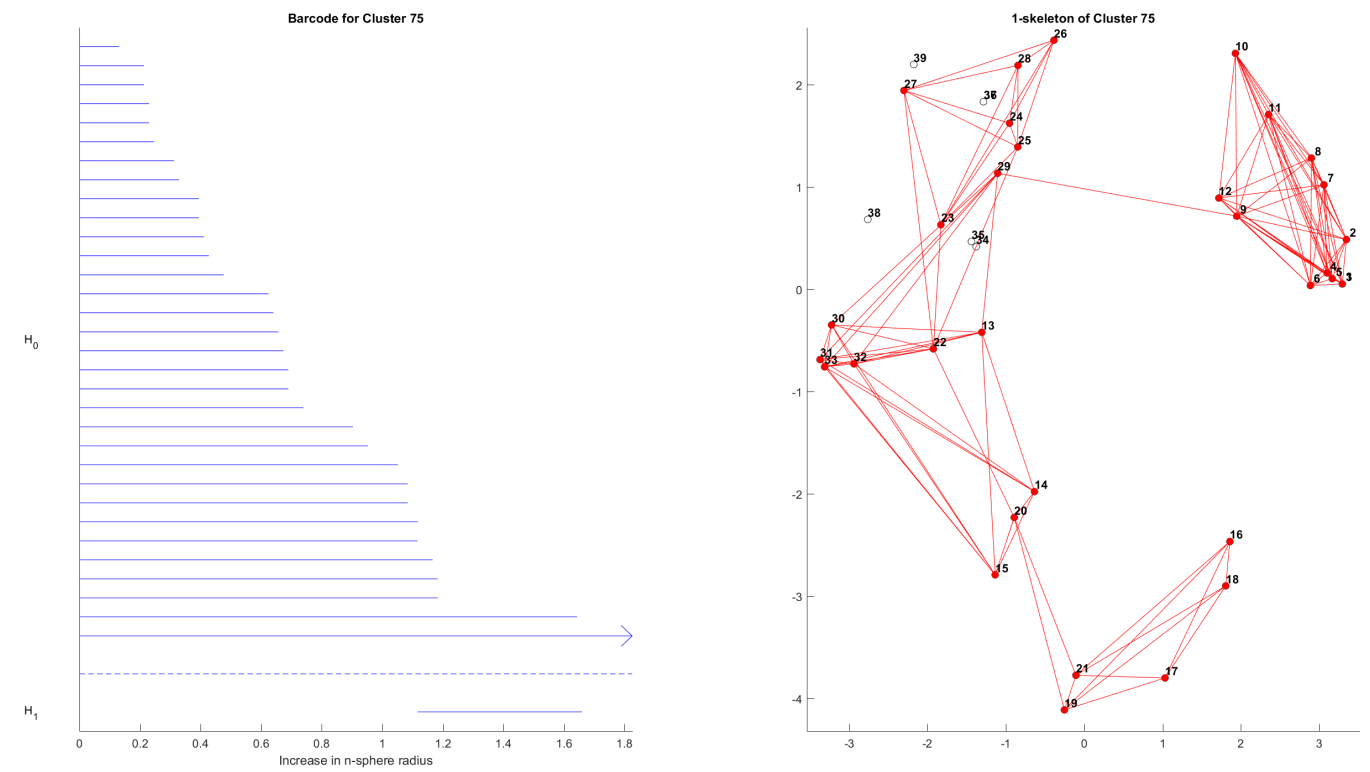

(A) Cluster N. 75
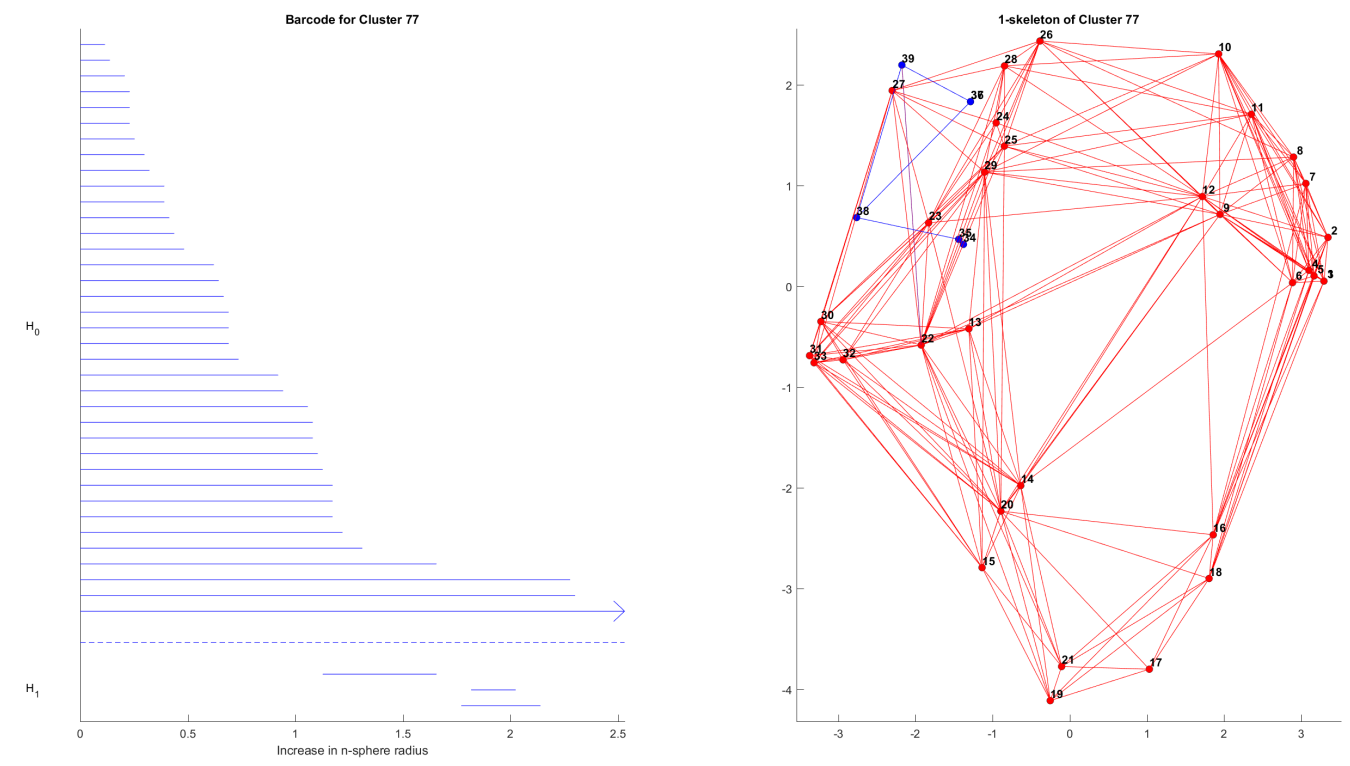

(B) Cluster N. 77

Figure 40. Persistent $H_{1}$-structures in the LanGeLin data, PCA $60 \%$, clusters N. 75 and N. 77. 

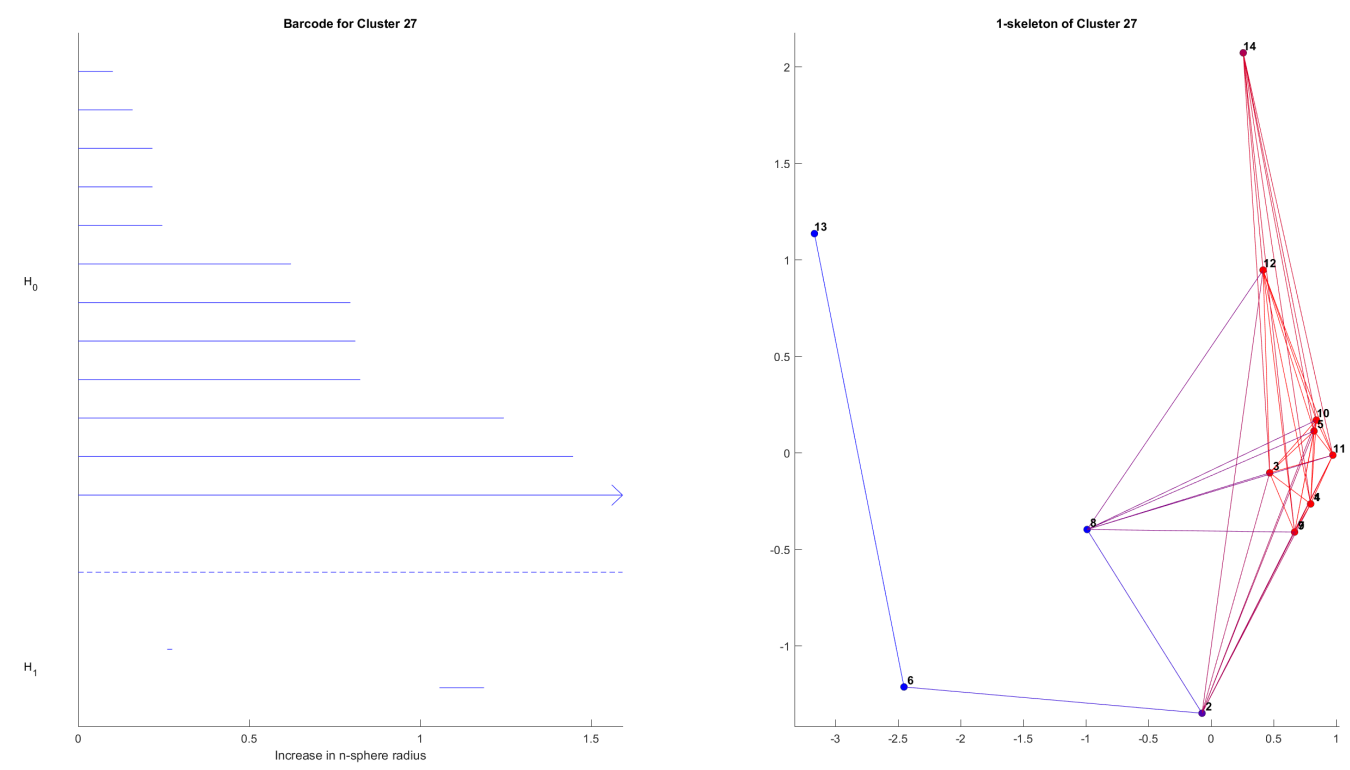

Figure 41 . Persistent $H_{1}$-generator in the Romance languages, LanGeLin data with PCA $60 \%$, cluster N. 27.

(1) identify the first cluster in the persistent components tree where the new $H_{1^{-}}$ generator appears,

(2) list the languages that are added in passing from the previous cluster (the last one without the new generator) to the new one,

(3) identify all the new cycles that are added in the 1-skeleton of the new cluster that were not present in the 1-skeleton of the previous cluster,

(4) in turn remove the languages belonging to one of the new cycles and recompute the persistent topology of the remaining set,

(5) check if the new $H_{1}$-generator is still present after the removal or not.

If more than one of the new cycles causes the disappearance of the $H_{1}$-generator then those cycles are homologous and either one can be chosen as generator. If the removal of a cycle does not cause the $H_{1}$-generator to disappear, then that cycle is a boundary in the VietorisRips complex and does not determine a non-trivial homology class. Another possibility, if the removal of a cycle does not eliminate the $H_{1}$-generator is that there are more than one homologous cycles that represent the same $H_{1}$-class and eliminating one of them will still leave another non-trivial homologous cycle. We will discuss these possibility in explicit examples below.

7.3. Example: homoplasy phenomena. One particularly interesting example is in cluster 141 of the SSWL Indoeuropean data (see Figure 7). The large cluster N. 140 has no significant $H_{1}$ generator. However, the language Brazilian Portuguese is added in going from cluster N. 140 to Cluster N. 141, and in doing so it makes 141 the smallest cluster with an $H_{1}$ generator of note. The natural question when looking at this example is what 

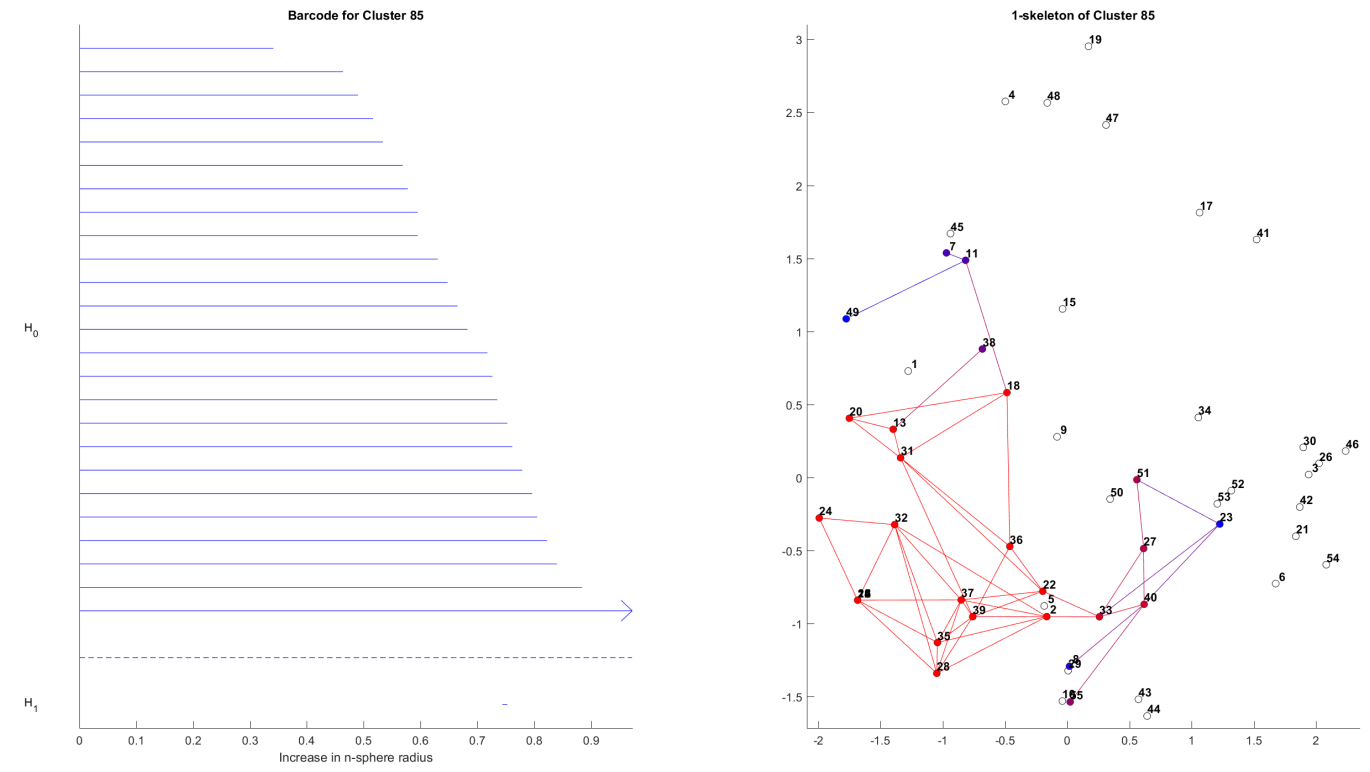

(A) Niger-Congo, cluster N. 85
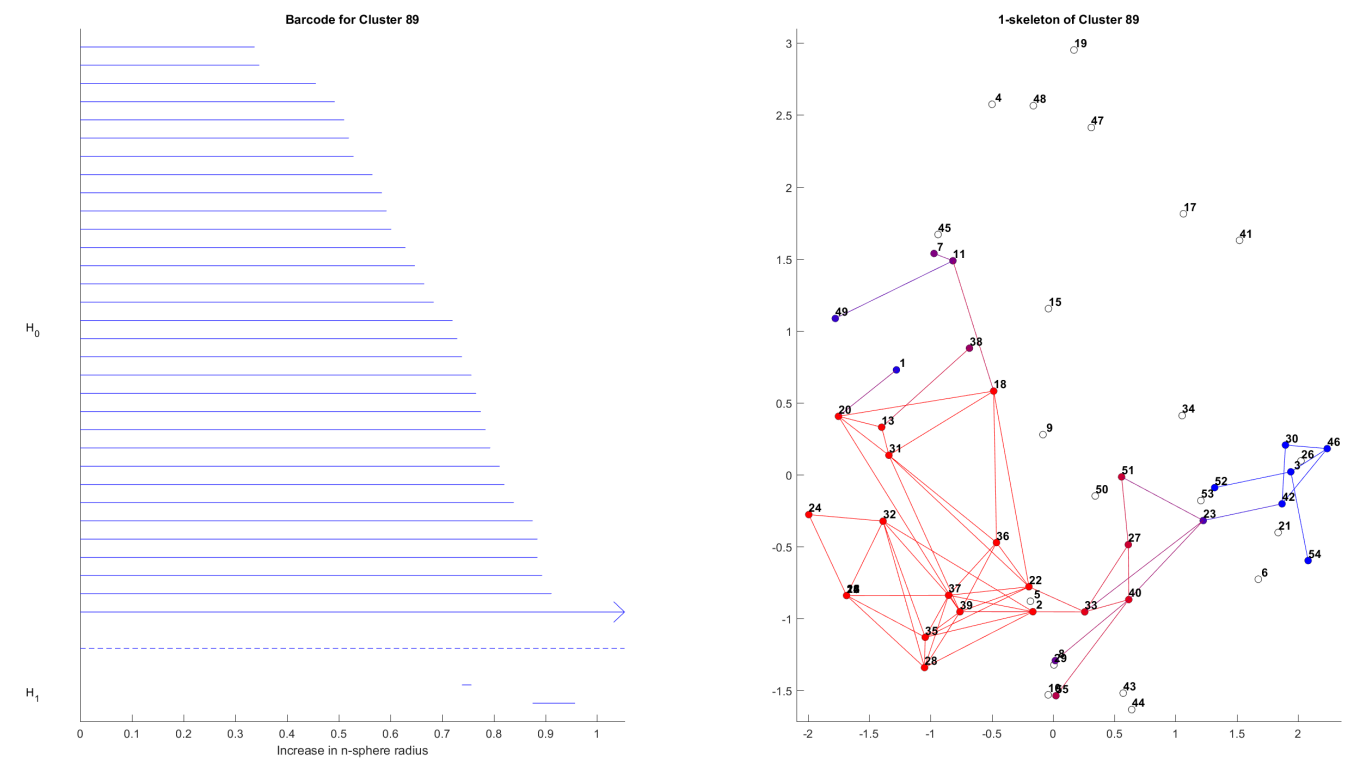

(B) Niger-Congo, cluster N. 89

Figure 42. Persistent $H_{1}$-structures in the Niger-Congo families, SSWL data, PCA 60\%, clusters N. 85 and N. 89. 

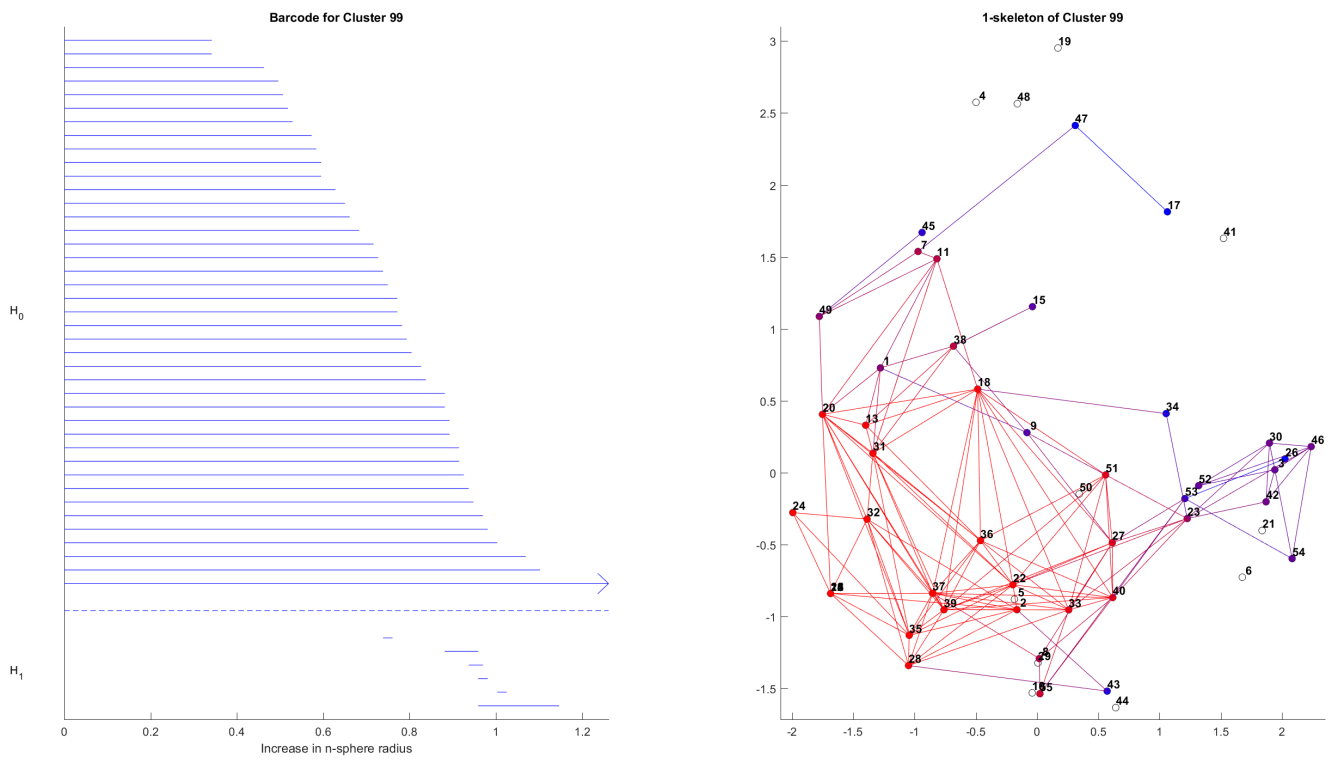

(A) Niger-Congo, cluster N. 99
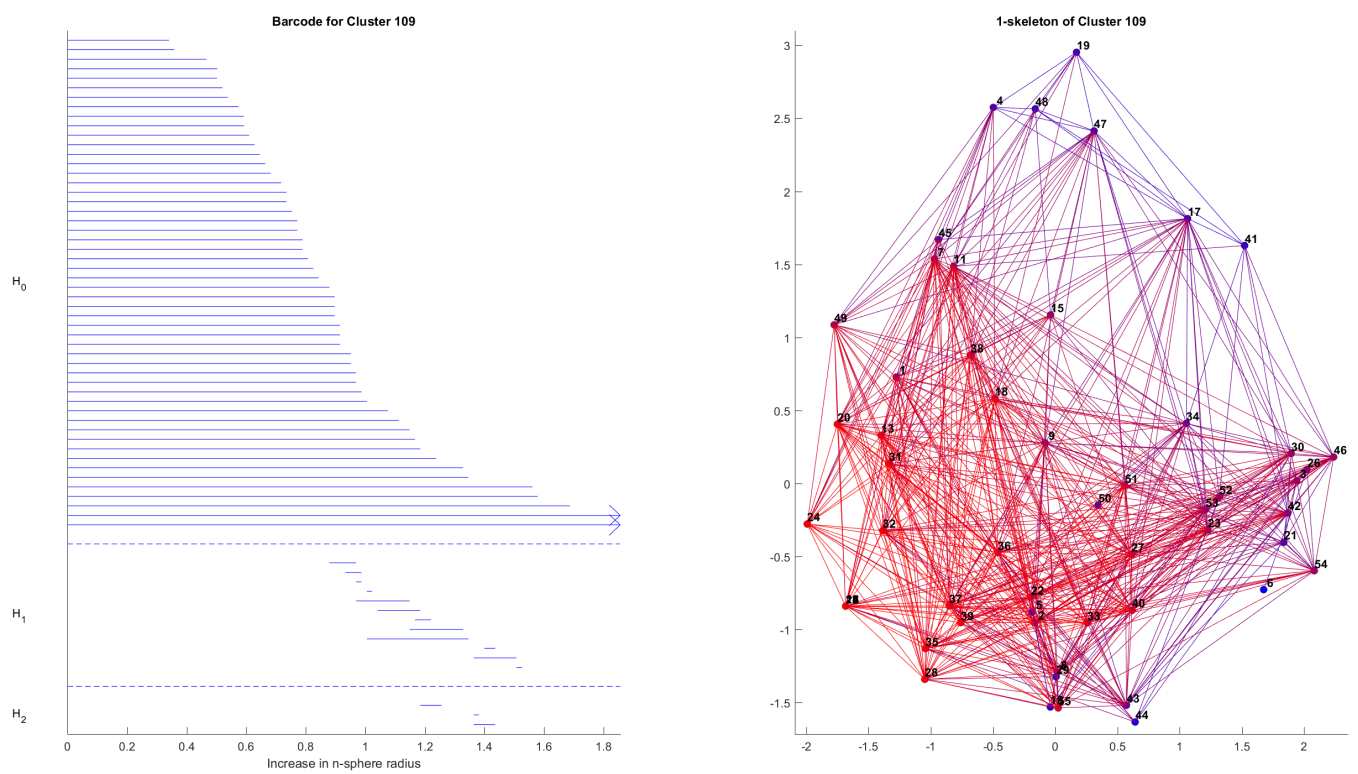

(в) Niger-Congo, cluster N. 109

Figure 43. Persistent $H_{1}$-structures in the Niger-Congo families, SSWL data, PCA 60\%, clusters N. 99 and N. 109. 

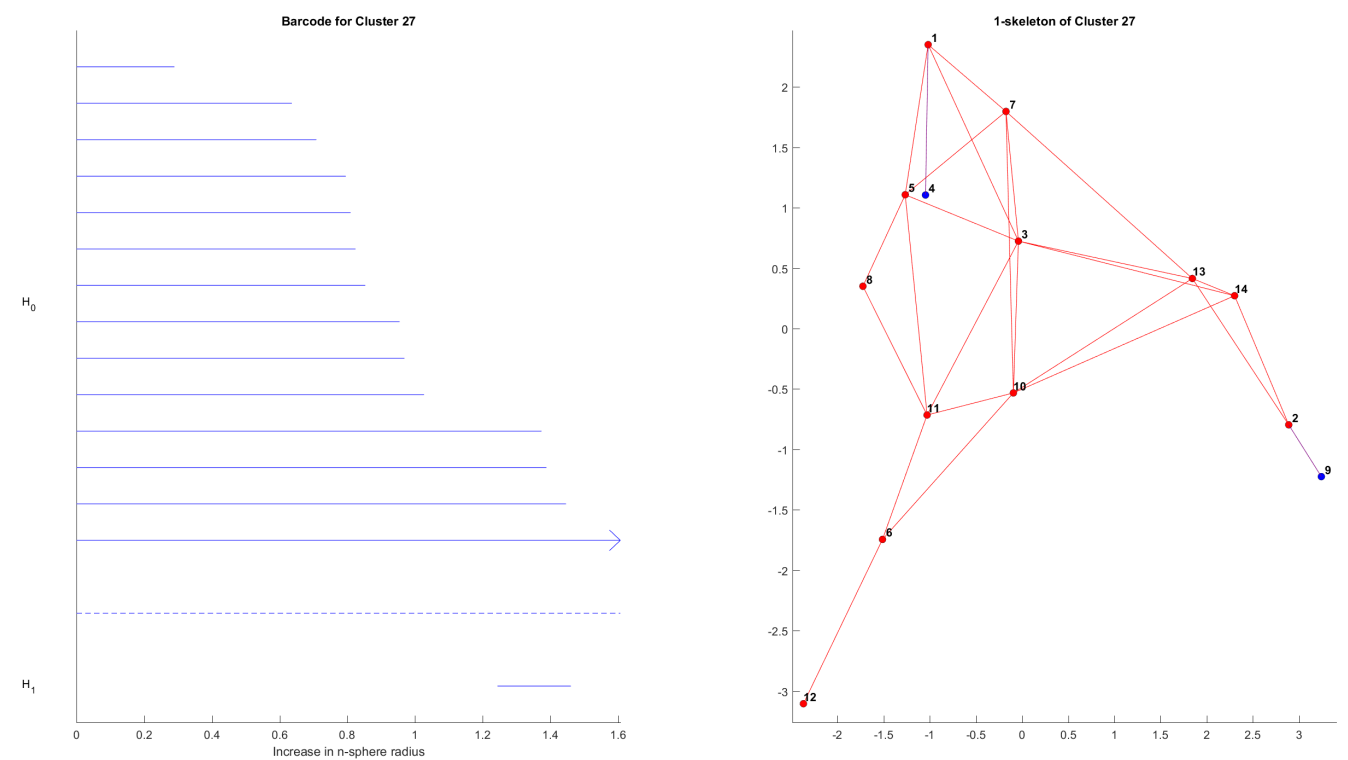

(A) Afro-Asiatic
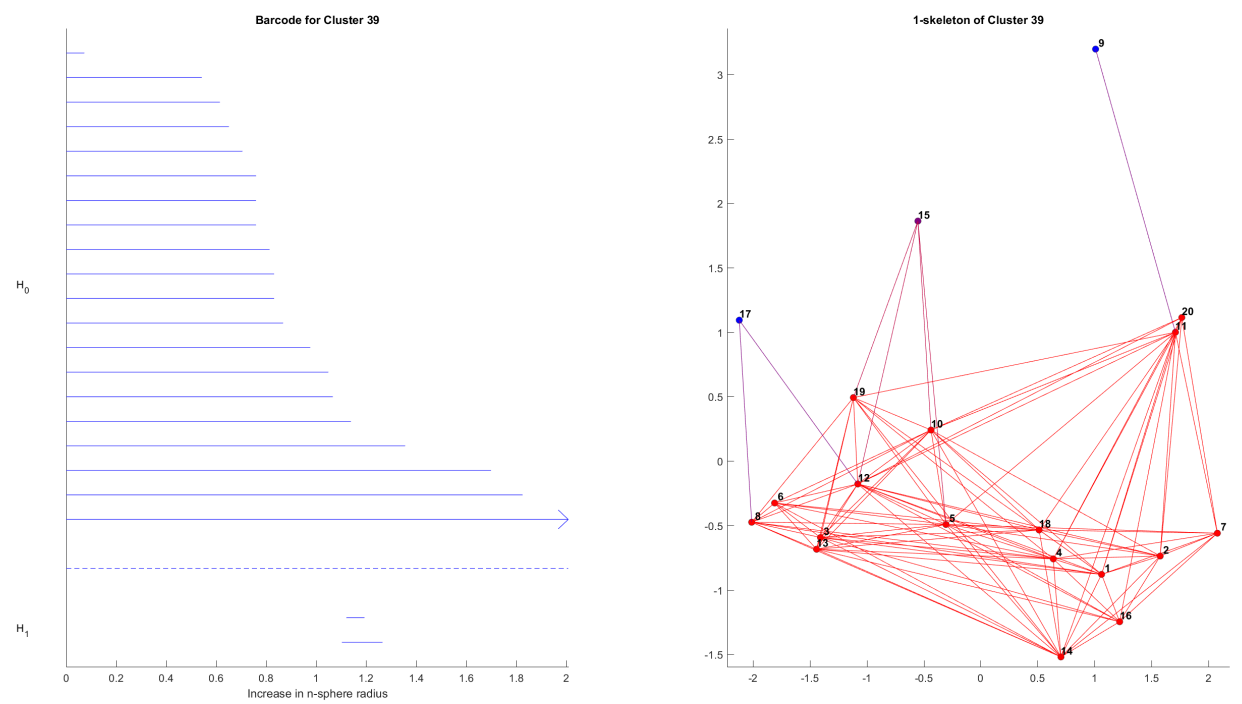

(B) Austronesian

Figure 44. Persistent topology in the Afro-Asiatic and Austronesian language families, SSWL data, PCA $60 \%$.

is the loop that gives the new non-trivial $H_{1}$-generator starting in Cluster N. 141 and how do we detect it. 
Using the method discussed above for identifying explicit $H_{1}$-generators, we see that the addition of the single new language Brazilian Portuguese in the transition from cluster N. 140 to cluster N. 141 causes a change to the 1-skeleton of the Vietoris-Rips complex, which consists of the addition of three new cycles: \{English, Swedish, European Portuguese\}, \{Czech, Lithuanian, Middle Dutch, Swiss German\} and \{Czech, Ukranian, Slovenian, Tocharian A\}. We remove in turn each one of these cycles from the data set and we compute the effect on the persistent $H_{1}$. Only in the case of the removal of the cycle consisting of $\{$ Czech, Lithuanian, Middle Dutch, Swiss German $\}$ the $H_{1}$-generator disappears, while it remains unchanged when removing one of the other two cycles. This means that the cycles \{English, Swedish, European Portuguese $\}$ and $\{$ Czech, Ukranian, Slovenian, Tocharian A\} are boundaries of 2-chains in the Vietoris-Rips complex, hence they do not define non-trivial homology classes, while the cycle \{Czech, Lithuanian, Middle Dutch, Swiss German $\}$ is not a boundary. This leads to the conclusion that this loop is a possible generator of the non-trivial $H_{1}$.

The Germanic languages Middle Dutch and Swiss German are closely related, but the fact that they occur in a non-trivial loop together with the Balto-Slavic Lithuanian and Czech seems difficult to justify in historical linguistic terms and is more likely representing a case of homoplasy detection.

7.4. Example: the Gothic-Slavic-Greek circle. We discuss another example of explicit identification of an $\mathrm{H}_{1}$-generator, with the same method discussed above, where the resulting generator may have a historical linguistic explanation beyond homoplasy.

The first persistent $H_{1}$-generator that we analyze in the Indo-European family, with the LanGeLin data, is illustrated in Figure 38 which shows the persistent generator arising between cluster N.102 and cluster N.113. The barcodes and the 1-skeleta are illustrated in the figure. Since only one $H_{1}$-generator is present most loops visible in the one skeleton are filled by 2 -simplices from the 2 -skeleton (not shown in the figures).

In order to find an explicit generator we remove some of the clusters involved and see which removals cause the $H_{1}$-generator to disappear. We observe that New Testament Greek, Romeyka Pontic Greek, and Gothic are in the loop generators, as removal of any one of them causes the generator to disappear. In [35] we had observed an $H_{1}$-generator in the Indo-European language family involving the Hellenic languages and some Slavic languages, hence we expect the Slavic languages to possibly play a role in this $H_{1}$-generator as well. We observe that the removal of any individual Slavic language does not cause the $H_{1}$-generator to disappear but the removal of all of them (which are very closely clustered together) destroys the persistent $H_{1}$-generator causing the appearance of a smaller one with a much shorter length of persistence in the barcode diagram. This suggests a geometry of a simplex where the three nodes New Testament Greek, Romeyka Pontic Greek, and Gothic are connected to some of the Slavic languages, which in turn are connected among themselves via 2-simplexes. This creates some homologous loops, so that the removal of a single Slavic language still leaves another nontrivial homologous generator while removal of the entire cluster of the Slavic languages removes it. The new smaller generator created by the removal of the Slavic languages accounts for the arrangement of 2-simplices around them.

In terms of historical linguistics the existence of a loop involving some of the Greek languages, Gothic, and some of the Slavic languages may be explainable in terms of a 
combination of historical phenomena. One is influences, here seen at the syntactic level, between the Greek languages and South Slavic languages, see for instance [30]. Another phenomenon that this non-trivial persistent $H_{1}$-generator may be capturing is the syntactic influence of New Testament Greek on Gothic, as discussed for instance in [12, where several calques from Greek constructions are identified in Gothic syntax. Finally, while it is known that the Gothic influence in the Proto-Slavic borrowing was primarily a lexical phenomenon (see [15], 28]), there is an indication of morpho-syntactic borrowing as well, see for instance [11. While we cannot be sure that the structure detected by the persistent topology is indeed reflecting these historical linguistic phenomena, this is a possible tentative explanation for the existence of this non-trivial loop and the languages involved.

7.5. Additional $H_{1}$-structures in the Indo-European family. The filtered SSWL data we considered in Section 6.3.2, for the Indo-European languages together with the hypothetical Ural-Altaic languages, show the first appearance of a very small (in terms of persistence interval) persistent $H_{1}$-generator at cluster $\mathrm{N}$. 78 , while two much more significant persistent $H_{1}$-generators arise at cluster N. 91, followed by a third one at the top cluster, N. 95, see Figure 39. We will discuss elsewhere the identification of explicit representative cycles for these $H_{1}$-generators.

By comparison, the LanGeLin data set, which comprises mostly Indo-European and Ural-Altaic languages, also has three non-trivial $H_{1}$-generators in the top cluster, $N .77$, with the first non-trivial one arising at cluster N. 75, and the other two at cluster N. 76, see Figure 40. In this case also we will return to discuss elsewhere explicit cycle generators for this $H_{1}$-structure, in comparison with those of the SSWL data.

The individual subfamilies of the Indo-European family do not show $H_{1}$-generators with long persistence, which is an indication that the three main persistent generators describe structures that simultaneously involve different subfamilies. Only the Romance languages in the filtered SSWL data show a small persistent $H_{1}$-generator in cluster N. 27, Figure 41, An explicit cycle representing this generator will be discussed elsewhere.

7.6. $H_{1}$-structures in other language families. In [35] where we only analyzed some of the sub-clusters of the various language families, we had not found significantly persistent $H_{1}$-structures in the sub-clusters we analyzed of the Niger-Congo languages. However, in the present analysis which we extended to all sub-clusters, we find that a first significant non-trivial persistent $H_{1}$-structure begins to occur in the Niger-Congo family at cluster N. 89, followed by a second significant persistent generator that arises at cluster N. 99 followed by more non-trivial $H_{1}$-generators at N. 109. The 1-skeleta and barcode diagrams for these clusters are shown in Figure 42 and Figure 43. A more in depth analysis of the $H_{1}$-structures in the Niger-Congo language family, identifying explicit generators and investigating their possible linguistic significance, will be conducted elsewhere.

The Austronesian language family exhibits two non-trivial $H_{1}$-generators in cluster N. 39 , see Figure 44. The Afro-Asiatic family shows only some occurrence of a single persistent $H_{1}$-generator which only arises in cluster N. 27, see Figure 44.

In these examples, at the stage where a new persistent $H_{1}$ generator arises, typically a few (sometimes just one) new languages are added to the cluster. This creates several new edges, which in turn add several new cycles to the 1-skeleton. We will return to a more detailed analysis of these cycles and an identification of an explicit generator among them. Note that, as in the case discussed in Section 7.3, the new languages added at the level 
where the new $H_{1}$ generator appears need not themselves be part of a cycle representative of the persistent $H_{1}$-generator.

\section{REFERENCES}

[1] M. Baker, The Atoms of Language, Basic Books, 2001.

[2] S.A. Barannikov, The Framed Morse complex and its invariants, Adv. Soviet Math. Vol.21 (1994) 93-115.

[3] M. Belkin, P. Niyogi, Laplacian eigenmaps for dimensionality reduction and data representation, Neural Comput. 15 (6) (2003) 1373-1396.

[4] J.D. Boissonnat, F. Chazal, M. Yvinec, Geometric and topological inference, Cambridge University Press, 2018.

[5] R. Bouckaert, P. Lemey, M. Dunn, S.J. Greenhill, A.V. Alekseyenko, A.J. Drummond, R.D. Gray, M.A. Suchard, Q.D. Atkinson, Mapping the origins and expansion of the Indo-European language family, Science, Vol.337 (2012) 957-960.

[6] P. Bubenik, J.A. Scott, Categorification of persistent homology, Discrete Comput. Geom. 51 (2014) no. 3, 600-627. arXiv:1205.3669

[7] G. Carlsson, Topology and Data, Bulletin of the American Mathematical Society, Vol. 46 (2009) N.2, $255-308$

[8] N. Chomsky, Lectures on Government and Binding, Dordrecht: Foris Publications, 1982.

[9] N. Chomsky, H. Lasnik, The theory of Principles and Parameters, in "Syntax: An international handbook of contemporary research", pp.506-569, de Gruyter, 1993.

[10] H. Edelsbrunner, J.L. Harer, Computational topology, American Mathematical Society, 2010.

[11] R. Genis, Comparing verbal aspect in Slavic and Gothic, in "Language for its own sake: essays on language and literature offered to Harry Perridon", Amsterdam contributions to Scandinavian studies; No. 8, (2012) 59-80.

[12] J.D. Gliesche, Gothic Syntax, lecture notes http://users.clas.ufl.edu/drjdg/oe/pubs/gothicsyntax.pdf

[13] C. Guardiano, D. Michelioudakis, A. Ceolin, M. Irimia, G. Longobardi, N. Radkevich, I. Sitaridou, G. Silvestri, South by Southeast. A Syntactic Approach to Greek and Romance Microvariation, L'Italia Dialettale, Vol. 77 (2016) 95-166.

[14] R. Ghrist, Elementary Applied Topology, CreateSpace, 2014.

[15] G. Holzer, Germanische Lehnwörter im Urslavischen: Methodologisches zu ihrer Identifizierung. Croatica, Slavica, Indoeuropea. Wien: Österreichischen Akademie der Wissenschaften. Series: Wiener Slawistisches Jahrbuch, Ergänzungsband; VIII (1990) 59-67.

[16] S. Karimi, M. Piattelli-Palmarini (Eds.), Special Issue on Parameters, Linguistic Analysis, Vol.41 (2017) N. 3-4.

[17] M. Kashiwara, P. Schapira, Persistent homology and microlocal sheaf theory, arXiv:1705.00955

[18] D. Kazakov, G. Cordoni, E. Algahtani, A. Ceolin, M. Irimia, S.S. Kim, D. Michelioudakis, N. Radkevich, C. Guardiano, G. Longobardi, Learning Implicational Models of Universal Grammar Parameters, EVOLANG XII: 16-19 April 2018, Torun, Poland.

[19] G. Longobardi, Methods in parametric linguistics and cognitive history, Linguistic Variation Yearbook 3 (2003) 101-138.

[20] G. Longobardi, Principles, Parameters, and Schemata. A constructivist UG, Linguistic Analysis, 41 (2017) 3-4, 517-557.

[21] G. Longobardi, A. Ceolin, The mathematics of parametric comparison, talk at workshop "Phylogenetic Linguistics and Linguistic Theory", York Centre for Linguistic History and Diversity, University of York, November 2018.

[22] G. Longobardi, C. Guardiano, Evidence for syntax as a signal of historical relatedness, Lingua, 119 (2009) 1679-1706.

[23] G. Longobardi, C. Guardiano, G. Silvestri, A. Boattini, A. Ceolin, Towards a syntactic phylogeny of modern Indo-European languages, Journal of Historical Linguistics, Vol.3 (2013) N.1, 122-152. 
[24] G. Longobardi, A. Buch, A. Ceolin, A. Ecay, C. Guardiano, M. Irimia, D. Michelioudakis, N. Radkevich, G. Jaeger. 2016. Correlated evolution or not? phylogenetic linguistics with syntactic, cognacy, and phonetic data, in (S.G. Roberts, et al., Eds.) "The Evolution of Language: Proceedings of the 11th International Conference (EVOLANGX11)", 2016 Online at http://evolang.org/neworleans/papers/162.html

[25] Yu.I. Manin, M. Marcolli, Nori diagrams and persistent homology, arXiv:1901.10301

[26] A. Marcantonio, The Uralic Language Family: Facts, Myths and Statistics, Publications of the Philological Society, Vol. 35, Blackwell, 2002.

[27] M. Marcolli, Syntactic Parameters and a Coding Theory Perspective on Entropy and Complexity of Language Families, Entropy, vol.18 (2016), N.4 110 [17pages].

[28] V.V. Martynov, Iazyk v prostranstve i vremeni, Nauka, 1983.

[29] A. Militarev, Genealogical classification of Afro-Asiatic languages according to the latest data, talk at the conference on the 70th anniversary of V.M. Illich-Svitych, Moscow, 2004.

[30] O. Mišeska-Tomić, Balkan Sprachbund. Morpho-syntactic Features, Dordrecht, Springer 2006.

[31] A. Ortegaray, R.C. Berwick, M. Marcolli, Heat Kernel analysis of syntactic structures, arXiv:1803.09832 [cs.CL]

[32] L. Pachter, B. Sturmfels, Algebraic Statistics for Computational Biology, Cambridge University Press, 2005.

[33] J.J.Park, R.Boettcher, A.Zhao, A.Mun, K.Yuh, V.Kumar, M.Marcolli, Prevalence and recoverability of syntactic parameters in sparse distributed memories, in "Geometric Science of Information. Third International Conference GSI 2017", pp.265-272, Lecture Notes in Computer Science, Vol.10589, Springer 2017.

[34] A. Perelysvaig, M.W. Lewis, The Indo-European controversy: facts and fallacies in Historical Linguistics, Cambridge University Press, 2015.

[35] A. Port, I. Gheorghita, D. Guth, J.M. Clark, C. Liang, S. Dasu, M. Marcolli, Persistent topology of syntax, Math. Comput. Sci. 12 (2018), no. 1, 33-50.

[36] D. Ringe, T. Warnow, A. Taylor, Indo-European and computational cladistics, Transactions of the Philological Society, Vol.100 (2002) 59-129.

[37] K. Shu, A. Ortegaray, R.C. Berwick, M. Marcolli, Phylogenetics of Indo-European language families via an algebro-geometric analysis of their syntactic structures, arXiv:1712.01719 [cs.CL]

[38] K. Shu, M. Marcolli, Syntactic structures and code parameters, Mathematics in Computer Science, 11 (2017), no. 1, 79-90.

[39] K. Shu, S. Aziz, V.L. Huynh, D. Warrick, M. Marcolli, Syntactic phylogenetic trees, in "Foundations of mathematics and physics one century after Hilbert", pp. 417-441, Springer, Cham, 2018.

[40] D. Sinor, The Problem of the Ural-Altaic relationship, in "The Uralic Languages: Description, History and Modern Influences", Brill 1988, pp. 706-741.

[41] K. Siva, J. Tao, M. Marcolli, Spin Glass Models of Syntax and Language Evolution, Linguistic Analysis, Vol.41 (2017) N.3-4, 559-608.

[42] A.J. Zomorodian, Topology for computing, Cambridge University Press, 2005.

[43] Perseus software package for persistent homology http://www . sas. upenn. edu/ vnanda/perseus/

[44] Syntactic Structures of World Languages (SSWL Database) http://sswl.railsplayground.net/

[45] Terraling Database http://test.terraling.com/

UNIVERSity OF SOUTHERN CALIFORNIA, USA

E-mail address: portam@usc.edu

California Institute of Technology, USA

E-mail address: tkaridi@caltech.edu

California Institute of Technology, USA

Perimeter Institute for Theoretical Physics, Canada

University of Toronto, CAnadA

E-mail address: matilde@caltech.edu 